Washington University School of Medicine Digital Commons@Becker

8-31-2021

\title{
Patient-specific iPSCs carrying an SFTPC mutation reveal the intrinsic alveolar epithelial dysfunction at the inception of interstitial lung disease
}

Konstantinos-Dionysios Alysandratos

Boston University

Jennifer A Wambach

Washington University School of Medicine in St. Louis

F Sessions Cole

Washington University School of Medicine in St. Louis

et al

Follow this and additional works at: https://digitalcommons.wustl.edu/open_access_pubs

Please let us know how this document benefits you.

\section{Recommended Citation}

Alysandratos, Konstantinos-Dionysios; Wambach, Jennifer A; Cole, F Sessions; and et al, "Patient-specific iPSCs carrying an SFTPC mutation reveal the intrinsic alveolar epithelial dysfunction at the inception of interstitial lung disease." Cell Reports. 36, 9. 109636 (2021).

https://digitalcommons.wustl.edu/open_access_pubs/10800

This Open Access Publication is brought to you for free and open access by Digital Commons@Becker. It has been accepted for inclusion in Open Access Publications by an authorized administrator of Digital Commons@Becker. For more information, please contact vanam@wustl.edu. 


\section{Cell Reports}

\section{Patient-specific iPSCs carrying an SFTPC mutation reveal the intrinsic alveolar epithelial dysfunction at the inception of interstitial lung disease}

\section{Graphical abstract}

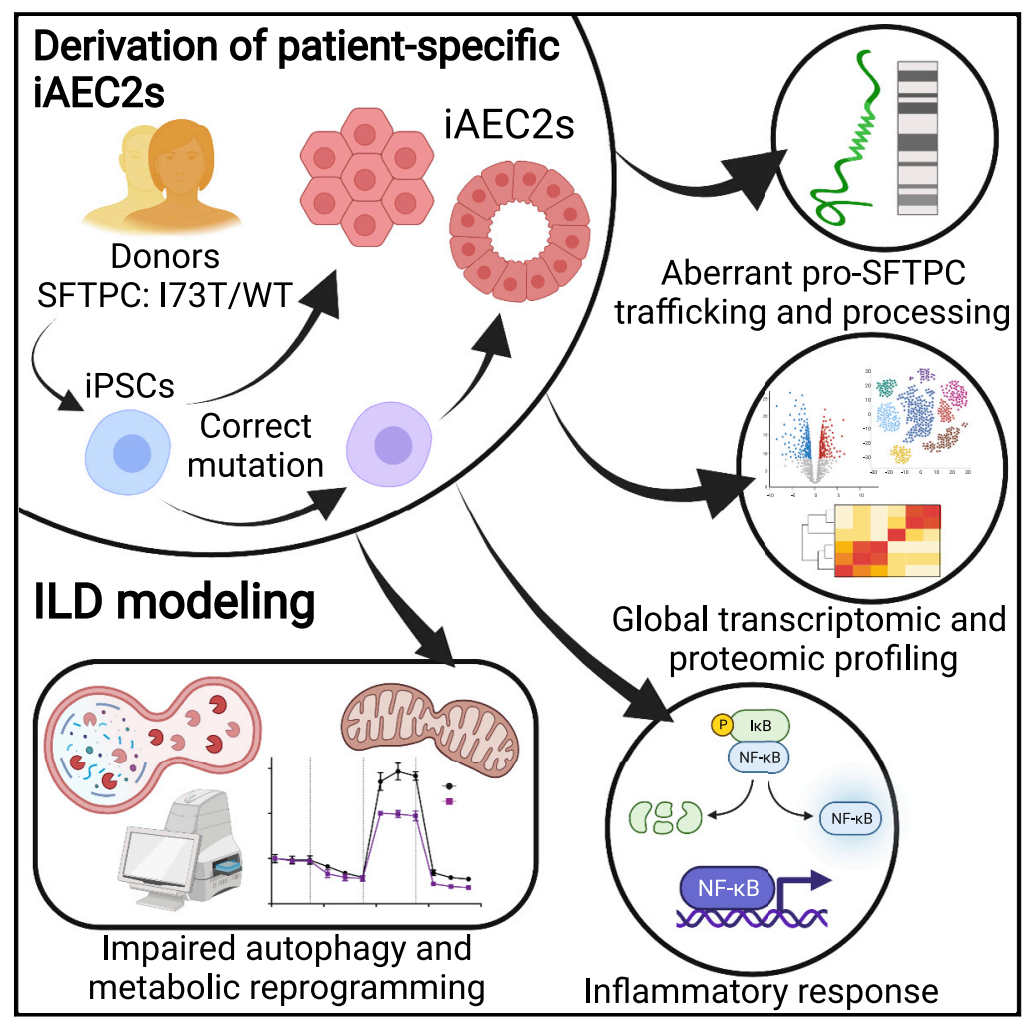

\section{Highlights}

- Patient-specific iAEC2s serve as a human preclinical platform for modeling ILD

- Patient-derived iAEC2s expressing mutant SFTPC ${ }^{173 T}$ have diminished progenitor capacity

- SFTPC ${ }^{173 T}$ mutant iAEC2s display proteostasis perturbations and metabolic reprogramming

- Mutant iAEC2s are an important proinflammatory hub via

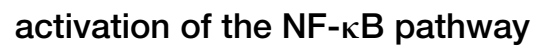

\section{Authors}

Konstantinos-Dionysios Alysandratos, Scott J. Russo, Anton Petcherski, ..., Orian S. Shirihai, Michael F. Beers, Darrell N. Kotton

\section{Correspondence}

mfbeers@pennmedicine.upenn.edu (M.F.B.), dkotton@bu.edu (D.N.K.)

\section{In brief}

Alysandratos et al. differentiate patientspecific iPSCs carrying the ILDassociated SFTPC ${ }^{173 T}$ variant and syngeneic corrected iPSCs into alveolar epithelial type 2 cells (iAEC2s). They find that mutant iAEC2s demonstrate proteostasis perturbations, altered bioenergetic programs, and metabolic reprogramming. They validate these findings in vivo in primary mouse Sftpc ${ }^{173 T}$ AEC2s. 


\title{
Patient-specific iPSCs carrying an SFTPC mutation reveal the intrinsic alveolar epithelial dysfunction at the inception of interstitial lung disease
}

\author{
Konstantinos-Dionysios Alysandratos, ${ }^{1,2}$ Scott J. Russo, ${ }^{3,4}$ Anton Petcherski, ${ }^{5}$ Evan P. Taddeo, ${ }^{5}$ Rebeca Acín-Pérez, ${ }^{5}$ \\ Carlos Villacorta-Martin, ${ }^{1}$ J.C. Jean, ${ }^{1,2}$ Surafel Mulugeta, ${ }^{3,4}$ Luis R. Rodriguez, ${ }^{3,4}$ Benjamin C. Blum, ${ }^{6}$ Ryan M. Hekman, ${ }^{6}$ \\ Olivia T. Hix, ${ }^{1}$ Kasey Minakin, ${ }^{1}$ Marall Vedaie,, ${ }^{1,2}$ Seunghyi Kook, ${ }^{7}$ Andrew M. Tilston-Lunel, ${ }^{8}$ Xaralabos Varelas, ${ }^{8}$ \\ Jennifer A. Wambach, ${ }^{9}$ F. Sessions Cole, ${ }^{9}$ Aaron Hamvas, ${ }^{10}$ Lisa R. Young, ${ }^{11}$ Marc Liesa, ${ }^{5}$ Andrew Emili, ${ }^{6}$ \\ Susan H. Guttentag, ${ }^{7}$ Orian S. Shirihai, ${ }^{5}$ Michael F. Beers, ${ }^{3,4,12, *}$ and Darrell N. Kotton ${ }^{1,2,12,13, *}$ \\ ${ }^{1}$ Center for Regenerative Medicine, Boston University and Boston Medical Center, Boston, MA 02118, USA \\ ${ }^{2}$ The Pulmonary Center and Department of Medicine, Boston University School of Medicine, Boston, MA 02118, USA \\ ${ }^{3}$ Pulmonary, Allergy, and Critical Care Division, Department of Medicine, University of Pennsylvania Perelman School of Medicine, \\ Philadelphia, PA 19104, USA \\ ${ }^{4}$ PENN-CHOP Lung Biology Institute, University of Pennsylvania Perelman School of Medicine, Philadelphia, PA 19104, USA \\ ${ }^{5}$ Departments of Medicine, Endocrinology and Molecular and Medical Pharmacology, David Geffen School of Medicine at UCLA, Los \\ Angeles, CA 90095, USA \\ ${ }^{6}$ Departments of Biology and Biochemistry, Boston University School of Medicine, Boston, MA 02118, USA \\ ${ }^{7}$ Department of Pediatrics, Monroe Carell Jr. Children's Hospital, Vanderbilt University, Nashville, TN 37232, USA \\ ${ }^{8}$ Departments of Biochemistry, Boston University School of Medicine, Boston, MA 02118, USA \\ 9Division of Newborn Medicine, Edward Mallinckrodt Department of Pediatrics, Washington University School of Medicine and St. Louis \\ Children's Hospital, St. Louis, MO 63110, USA \\ 10Department of Pediatrics, Northwestern University Feinberg School of Medicine, Chicago, IL 60611, USA \\ ${ }^{11}$ Division of Pulmonary and Sleep Medicine, Department of Pediatrics, Children's Hospital of Philadelphia, Philadelphia, PA 19104, USA \\ 12 Senior author \\ ${ }^{13}$ Lead contact \\ *Correspondence: mfbeers@pennmedicine.upenn.edu (M.F.B.), dkotton@bu.edu (D.N.K.) \\ https://doi.org/10.1016/j.celrep.2021.109636
}

\section{SUMMARY}

Alveolar epithelial type 2 cell (AEC2) dysfunction is implicated in the pathogenesis of adult and pediatric interstitial lung disease (ILD), including idiopathic pulmonary fibrosis (IPF); however, identification of disease-initiating mechanisms has been impeded by inability to access primary AEC2s early on. Here, we present a human in vitro model permitting investigation of epithelial-intrinsic events culminating in AEC2 dysfunction, using patient-specific induced pluripotent stem cells (iPSCs) carrying an AEC2-exclusive disease-associated variant $\left(S F T P C^{173 T}\right)$. Comparing syngeneic mutant versus gene-corrected iPSCs after differentiation into AEC2s (iAEC2s), we find that mutant iAEC2s accumulate large amounts of misprocessed and mistrafficked pro-SFTPC protein, similar to in vivo changes, resulting in diminished AEC2 progenitor capacity, perturbed proteostasis, altered bioenergetic programs, time-dependent metabolic reprogramming, and nuclear factor $\kappa B(N F-\kappa B)$ pathway activation. Treatment of SFTPC ${ }^{173 T}$-expressing iAEC2s with hydroxychloroquine, a medication used in pediatric ILD, aggravates the observed perturbations. Thus, iAEC2s provide a patient-specific preclinical platform for modeling the epithelial-intrinsic dysfunction at ILD inception.

\section{INTRODUCTION}

Idiopathic pulmonary fibrosis (IPF) is the most common and severe form of idiopathic interstitial pneumonia and is characterized by relentless fibrosis leading to disruption of the gas exchange unit and death within an average of 4 years from the time of diagnosis (Lederer and Martinez, 2018; Raghu et al., 2014, 2016; Travis et al., 2013). The poorly understood pathogenesis of IPF, in part due to the lack of human disease models, has been a major hurdle in developing effective therapies. Although a broad, established literature has focused on the role of lung fibroblasts in perpetuating IPF at later disease stages, factors that initiate or drive disease onset at early stages have been particularly hard to identify. With the advent of genome-wide association studies and intensive study of familial forms of pulmonary fibrosis, the lung epithelium has been increasingly implicated as a potential proximal disease driver with variants in gene loci expressed in lung epithelia having been associated with disease risk (Garcia, 2018; Kropski et al., 2015).

Of the many types of epithelia present in the lung, dysfunction of the alveolar epithelial type 2 cell (AEC2), in particular, has been 
repeatedly implicated in the pathogenesis of interstitial lung disease (ILD), including IPF (Barkauskas and Noble, 2014; Katzenstein, 1985; Winters et al., 2019; Selman and Pardo, 2014). Determining how AEC2 dysfunction leads to disease in humans, however, has been challenging to date. For example, studying AEC2 pathophysiology in primary human cells has been limited by difficulty in accessing patient samples and the tendency of AEC2s to lose their identity in culture (Borok et al., 1998; Foster et al., 2007), particularly in the absence of mesenchymal feeders (Barkauskas et al., 2013).

An ideal model of human AEC2 dysfunction would allow the study of disease by utilizing patient-derived cells to reveal a cascade of mechanistic events associated with the inception as well as progression of lung disease. Here, we present such a model of disease inception employing induced pluripotent stem cells (iPSCs) that we have derived from patients with ILD in order to generate an inexhaustible supply of AEC2-like cells in vitro to study the epithelial-intrinsic events that lead to specific AEC2 perturbations that are then validated in one of the donor patient's tissue in vivo. To ensure examination of only the cell type responsible for initiating disease without the potential confounding or secondary effects of co-cultured supporting cells, we have developed an epithelial-only model composed solely of purified AEC2-like cells that can be propagated indefinitely without any supporting cells, and we have chosen to focus here on a disease-associated variant in the surfactant protein C (SFTPC) gene because it is known to be expressed postnatally solely in AEC2s and can be gene-edited to provide syngeneic comparator cells, thus providing a reductionist model amenable to discerning the putative epithelial-intrinsic events associated with disease inception.

Heterozygous mutations in SFTPC have been associated with both sporadic and familial pulmonary fibrosis as well as childhood interstitial lung disease (chILD) (Cottin et al., 2011; Crossno et al., 2010; Mulugeta et al., 2015; Nogee et al., 2001; Ono et al., 2011; Thomas et al., 2002; van Moorsel et al., 2010). Among the more than 60 SFTPC variants described to date, the missense mutation g.1286T $>\mathrm{C}$, resulting in substitution of threonine for isoleucine at amino acid 73 in the pro-SFTPC protein $\left(\right.$ SFTPC $\left.^{173 T}\right)$, is the focus of our study because it is the predominant disease-associated SFTPC variant. Two recent mouse genetic models, conditionally expressing mutant SFTPC (p.I73T and p.C121G, respectively) from the endogenous Sftpc locus, resulted in polycellular alveolitis and pulmonary fibrosis (Katzen et al., 2019; Nureki et al., 2018). Although previous studies have shown that expression of SFTPC ${ }^{173 T}$ in vitro (Beers et al., 2011; Hawkins et al., 2015) or in mice in vivo (Nureki et al., 2018) leads to SFTPC proprotein misprocessing and lung fibrosis, the cellular mechanisms by which dysfunctional AEC2s initiate the fibrotic cascade in humans remain elusive. Most importantly, elucidating the molecular pathogenesis of human AEC2 dysfunction caused by SFTPC ${ }^{173 T}$ is likely to inform the broader mechanisms by which AEC2 dysfunction leads to IPF.

A critical role for AEC2s in ILD initiation is concordant with their essential role in maintaining distal lung homeostasis. AEC2s function both as facultative progenitors of alveoli as well as secretory cells in the distal lung. In order to meet the high meta- bolic demands posed by these functions, AEC2s have a larger number of mitochondria compared to other lung cell types (Massaro et al., 1975), and alterations in mitochondrial function, as observed in our model, are likely to lead to a variety of perturbed metabolic programs. Indeed, accumulation of dysmorphic and dysfunctional mitochondria in association with impaired autophagy and mitophagy has been shown in AEC2s from IPF lungs (Bueno et al., 2015). Furthermore, thyroid hormone-mediated restoration of mitochondrial function and mitophagy was recently shown to blunt pulmonary fibrosis in two mouse models of pulmonary fibrosis (Yu et al., 2018).

Because there are significant differences between murine and human pulmonary fibrosis, there is a pressing need for reliable human preclinical disease models that would not only provide further insight into the pathophysiology of human AEC2 dysfunction at ILD inception, but would also serve as a platform to test the safety and efficacy of unproven currently used as well as novel therapies. Here, we show that SFTPC $C^{173 T}$-expressing human iPSC-derived AEC2s (hereafter iAEC2s) provide further insights into disease pathogenesis, allow assessment of currently used unproven treatments, and have the potential to serve as a platform for testing novel therapeutics.

\section{RESULTS}

Generation of patient-specific iPSC lines and their differentiation to alveolar epithelium

To examine the mechanisms leading to human AEC2 dysfunction, we generated patient-specific iPSC lines by reprogramming dermal fibroblasts (SPC2) or peripheral blood mononuclear cells (SPC6 and SPC7) from three individuals with lung disease who were heterozygous for the most frequent disease-associated SFTPC variant (SFTPC ${ }^{173 T / W T}$ ) (Figure 1A; Table S1). The SPC2 donor in particular suffered from severe ILD (Figure 1B and detailed clinical phenotyping in Table S1) necessitating the need for lung transplantation in early adolescence providing access to histopathological specimens of diseased explanted lung tissue (Figure 1C). The SPC6 and SPC7 donors also suffered from ILD based on chest computed tomography (CT) imaging (Figure S1A; Table S1). To overcome the hurdles imposed by the high variance in differentiation efficiencies or cellular phenotypes associated with different genetic backgrounds of iPSC lines generated from distinct individuals (Bock et al., 2011; Boulting et al., 2011; Kim et al., 2010) and to allow for purification of specific cell types, we engineered gene-corrected syngeneic lines by using activator-like effector nucleases (TALEN)-based gene editing to knock in a tdTomato fluorescent reporter into one allele of the endogenous SFTPC locus of the parental SPC2 iPSC line. This resulted in generation of syngeneic cor-

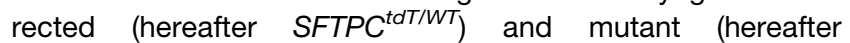
SFTPC $^{173 T / t d T}$ ) iPSC lines, because the tdTomato cassette is followed by a stop/polyA cassette, preventing expression of the subsequent SFTPC coding sequence from the targeted allele (Figure 1D). Thus, this targeting strategy was designed to result in preparation of a reductionist model where only one SFTPC allele would be expressed in each paired clone, enabling study of only the mutant versus only the normal allele. It also allowed tracking of SFTPC ${ }^{\text {tdTomato+ }}$ cells as they emerge through 
A

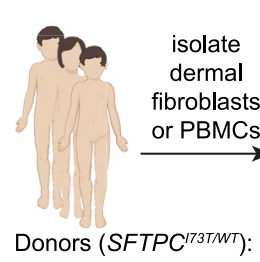

SPC2, SPC6, SPC7
B

Chest CT

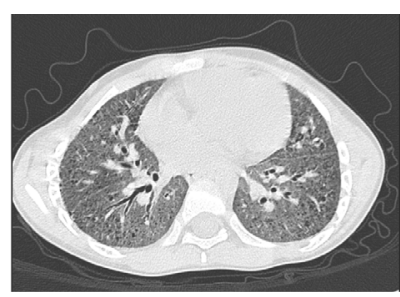

C

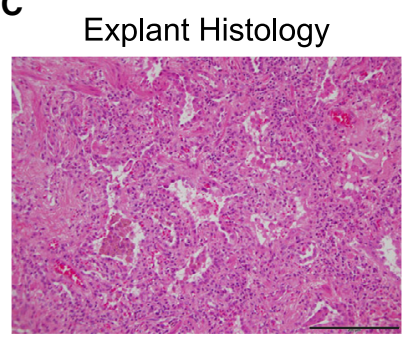

D

SFTPC locus

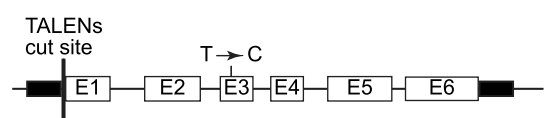

$\mathrm{E1}-\mathrm{E2}-\mathrm{E} 3-\mathrm{E} 4-\mathrm{E} 5-\mathrm{E} 6$
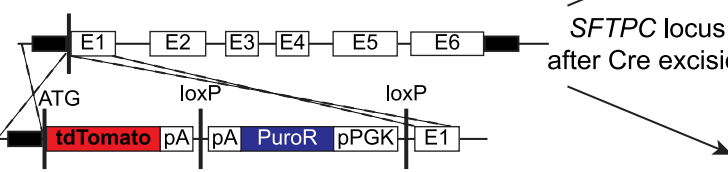

Donor template targeted into locus after Cre excision

tdT/WT

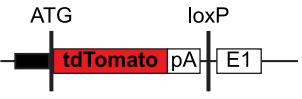

$-\mathrm{E1}-\mathrm{E2}-\mathrm{E3}-\mathrm{E4}-\mathrm{E} 5-\mathrm{E} 6 \mathrm{-}$

173T/tdT

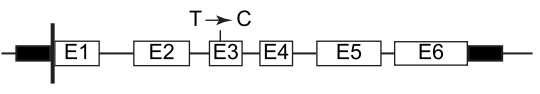

$\stackrel{\text { ATG }}{\text { AT }}$

E

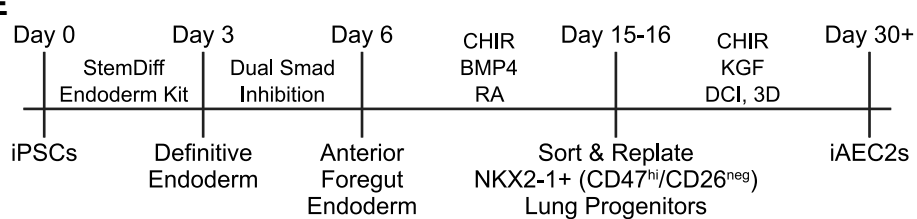

F

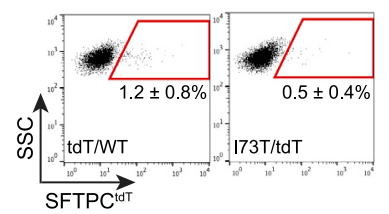

Day 30+

CHIR withdrawal and addback

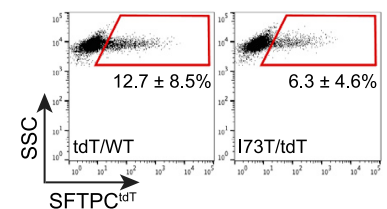

Day +14

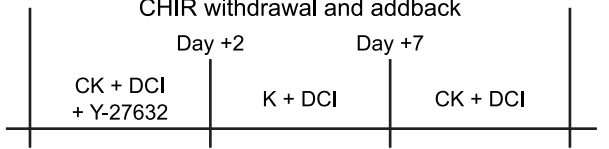

Sort \& Replate

Passage iAEC2s

as single cells

SFTPC tdomato+ $^{\text {iAEC2s }}$

G

Expression threshold

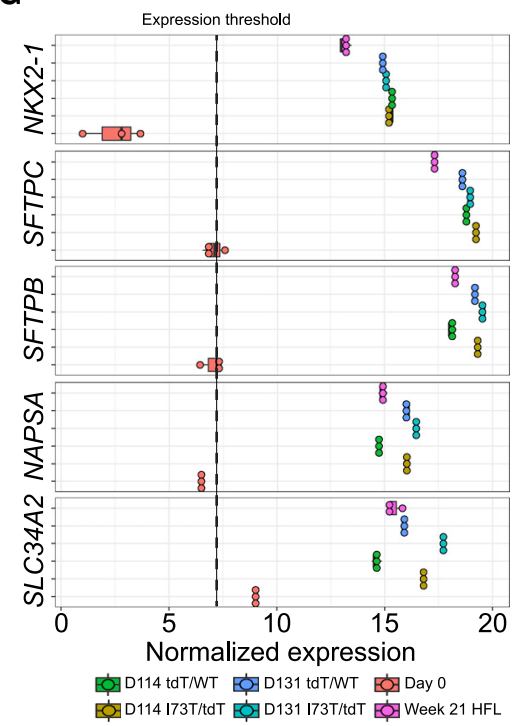

Figure 1. Generation of patient-specific iPSC lines and their differentiation to alveolar epithelium

(A) Schematic showing the generation of patient-specific iPSCs from dermal fibroblasts or peripheral blood mononuclear cells (PBMCs) from three individuals carrying the most frequent SFTPC pathogenic variant (SFTPC ${ }^{173 T / W T}$ ).

(B) Chest CT of the SPC2 donor reveals diffuse ground glass opacities and traction bronchiectasis.

(C) H\&E staining of the SPC2 donor lung explant shows end-stage lung disease with interstitial fibrosis, chronic inflammation, and alveolar remodeling with AEC2 hyperplasia and degenerating macrophages within the residual alveoli. Scale bar, $200 \mu \mathrm{m}$.

(D) Transcription activator-like effector nucleases (TALEN) targeting strategy and edited SFTPC loci post Cre-mediated antibiotic cassette excision.

(E) Schematic of directed differentiation protocol from iPSCs to day 30+ monolayered epithelial iAEC2 spheres (alveolospheres).

(F) Schematic of CHIR modulation to achieve iAEC2 maturation and representative flow cytometry dot plots (mean \pm SD; $\mathrm{n}=6$ biological replicates of independent differentiations).

(G) Dot plots depicting the normalized expression level of AEC2-marker genes in day 114 and day 131 SFTPC ${ }^{\text {tdT/WT }}$ and SFTPC ${ }^{173 T / t d T}$ iAEC2s compared to day 0 iPSCs and week 21 human fetal distal lung (HFL) controls, by bulk RNA sequencing (boxplots represent mean \pm SD; $n=3$ experimental replicates of independent wells of a differentiation). 
A
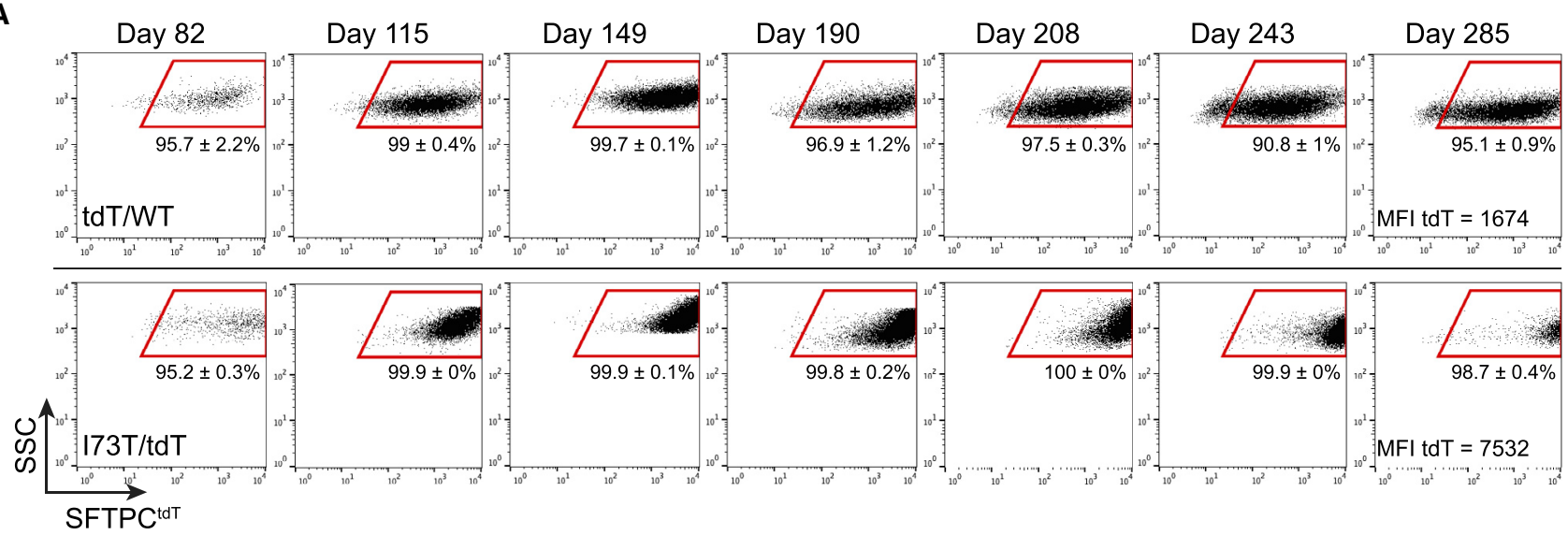

B

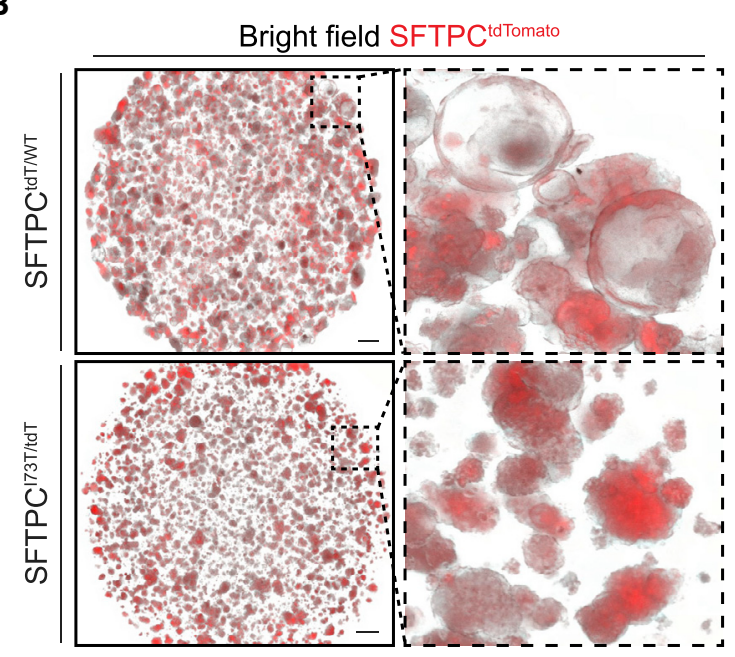

D

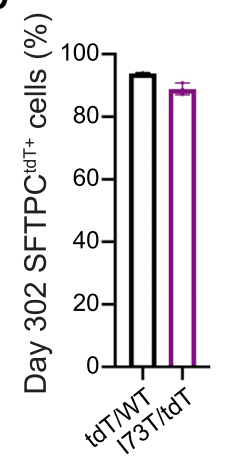

C

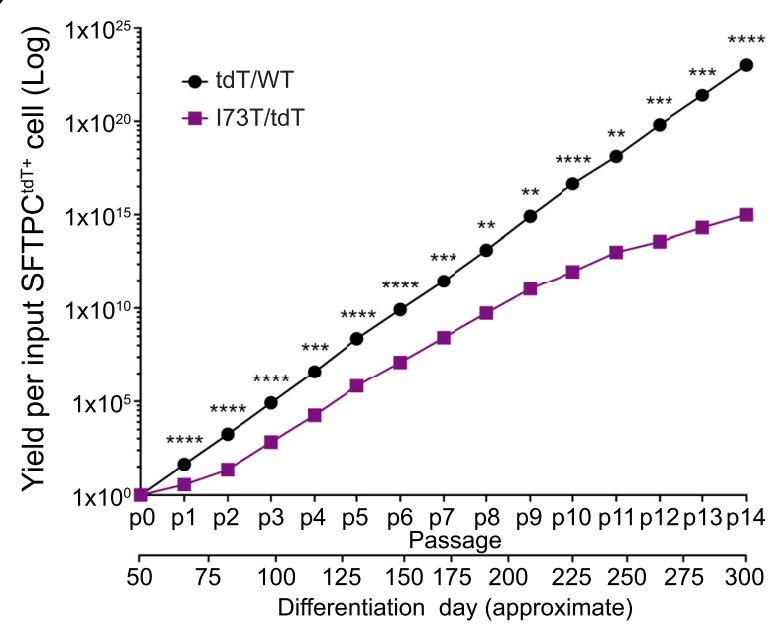

$\mathbf{F}$

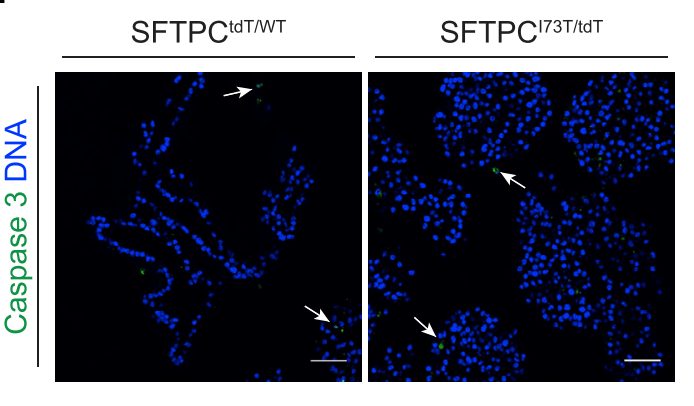

Figure 2. Derivation of parallel self-renewing corrected (SFTPC ${ }^{\text {tdT/WT }}$ ) and mutant (SFTPC ${ }^{173 T / t d T}$ ) iAEC2s

(A) Representative flow cytometry dot plots of day 82 (p2), day 115 (p4), day 149 (p6), day 190 (p8), day 208 (p9), day 243 (p11), and day 285 (p13) SFTPC ${ }^{\text {tdT/WT }}$ and SFTPC ${ }^{173 T / d T}$ iAEC2s. MFI, mean fluorescence intensity.

(B) Representative live-cell imaging of SFTPC ${ }^{\text {tdT/WT }}$ and SFTPC ${ }^{173 T / t d T}$ alveolospheres (bright-field/tdTomato overlay; day 149 ). Scale bars, $500 \mu \mathrm{m}$.

(C) Graph showing yield per input tdT/WT or I73T/tdT SFTPC ${ }^{\text {tdTomato+ }}$ cell sorted on day 51 and passaged without further sorting. ${ }^{\star *} \mathrm{p}<0.01,{ }^{* \star \star} \mathrm{p}<0.001,{ }^{* \star \star *} \mathrm{p}<$ 0.0001 by unpaired, two-tailed Student's $t$ test.

(D) Bar graph shows retention of the AEC2 cell fate in SFTPC tdT/WT and SFTPC ${ }^{173 T / \text { tdT }}$ iAEC2s maintained in culture for 302 days, measured by flow cytometry as the frequency of cells expressing the SFTPC ${ }^{\text {tdTomato }}$ reporter. 
in vitro directed differentiation and purification of corrected and mutant SFTPC ${ }^{\text {tdTomato+ }}$ putative iAEC2s for subsequent profiling.

To ensure that any findings from this single allele expression model could be replicated in iAEC2s expressing two active SFTPC alleles, as occurs in vivo, we also engineered an untargeted clone of the SPC2 line (hereafter SFTPC ${ }^{173 T / W T}$ ), which then underwent footprint-free correction of the SFTPC variant via CRISPR/Cas9 gene editing (hereafter SFTPC ${ }^{W T / W T}$ ) (Figure S1B). Furthermore, to ensure reproducibility across different genetic backgrounds, we utilized the other two donor iPSC lines (SPC6 and SPC7; clinical phenotyping in the Supplemental information) to validate key observations made in the SPC2 iPSC lines.

Derivation of parallel indefinitely self-renewing iAEC2s Next, we used our previously published lung directed differentiation protocol (Jacob et al., 2017, 2019) to generate corrected and mutant iAEC2s (Figures $1 \mathrm{E}$ and 1F), with the goal of producing indefinitely self-renewing patient-specific cells for disease modeling. Corrected and mutant SFTPC ${ }^{\text {tdTomato+ }}$ iAEC2s were sorted to purity for further replating, characterization of gene expression (Figure 1G), and culture expansion as self-renewing epithelial-only spheres without the need for subsequent cell sorting (Figures $2 \mathrm{~A}$ and $2 \mathrm{~B}$ ). The resulting iAEC2s maintained an AEC2-specific transcriptomic profile (Figure 1G); however, mutant iAEC2s demonstrated distinct morphologies and brighter tdTomato reporter expression (Figures $2 \mathrm{~A}$ and $2 \mathrm{~B}$ and further discussed below).

We sought to determine both the stability of SFTPC expression levels as well as the total cell yield arising during serial passaging of mutant versus corrected iAEC2s in epithelialonly 3D cultures. Over a 302-day period, we generated yields of $>10^{23}$ and $>10^{15}$ corrected versus mutant iAEC2s per starting sorted tdTomato ${ }^{+}$cell, respectively (Figure $2 \mathrm{C}$ ). Although yields over this time period were significantly lower for mutant iAEC2s, both lines maintained SFTPC ${ }^{\text {tdTomato }}$ expression in $94 \% \pm 0.3 \%$ and $89.1 \% \pm 1.9 \%$ of cells for at least 302 days and maintained a normal karyotype until at least day 214 (Figure 2D). In this self-renewing model, mutant iAEC2s demonstrated significantly lower proliferation rates than corrected iAEC2s assessed by EdU incorporation assays (Figure 2E) and no differences in apoptosis, as measured by immunostaining for activated caspase-3 (Figure 2F). These results suggest SFTPC mutant iAEC2s have diminished self-renewal capacity.

SFTPC ${ }^{173 T}$ iAEC2s display altered apical-basal polarity and aberrant protein trafficking and accumulate large amounts of aberrantly processed SFTPC pro-protein We further examined the impact of the SFTPC ${ }^{173 T}$ mutation on AEC2 morphology in vitro and in vivo. Although corrected

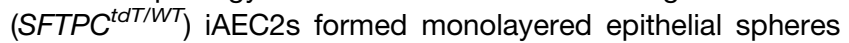
(alveolospheres) in culture as expected (Jacob et al., 2017), mutant (SFTPC ${ }^{173 T / t d T}$ ) iAEC2s lacked lumens and instead formed ball-like structures when assessed by microscopy of paraffin tissue sections (Figure 3A, panel i), semi-thin plastic sections (Figure 3A, panel ii), or epithelial cell adhesion molecule (EPCAM) immunostained sections (Figure 3B). Similarly, we found morphologic changes in developing $A E C 2 s$ in vivo in Sftpc $^{173 T}$ knockin mice, which do not survive birth (Nureki et al., 2018). For example, we examined E18.5 lungs from these mice and found they displayed arrested lung morphogenesis in late sacculation and complete obliteration of primordial saccules by clumps of HA-tagged pro-SFTPC ${ }^{173 T}$ expressing cells (Figure $3 \mathrm{C}$ ). Most importantly, analysis of the SPC2 donor lung explant also revealed distal lung morphological changes with areas of hyperplastic or clumped AEC2s that differed from normal lung tissue control sections (Figure 3D; arrowheads), further validating the observations made in vitro in the human iAEC2s. Furthermore, analysis of the same patient lung sections for the AEC1 marker RAGE revealed paucity of AEC1s (Figure S2A), similar to findings described in Sftpc ${ }^{173 T}$ knockin mice (Nureki et al., 2018), suggesting disruption of AEC2/AEC1 cell homeostasis as a result of AEC2 dysfunction.

To screen for potential morphological differences in lamellar bodies between mutant and corrected iAEC2s, we employed toluidine blue-stained plastic sections (Figure $3 \mathrm{~A}$, panel ii) as well as transmission electron microscopy (TEM) (Figure 3E). We observed small inclusions reminiscent of lamellar bodies, present intracellularly in both corrected and mutant iAEC2s (Figure $3 \mathrm{~A}$ panel ii; arrowheads) and extracellularly in the lumens of corrected alveolospheres, possibly reflecting lamellar body exocytosis (Figure 3A panel ii; ellipse), in keeping with our prior publication characterizing surfactant secretion from iAEC2s (Jacob et al., 2017). TEM confirmed these inclusions to be lamellar bodies (Figure 3E), with no appreciable difference in lamellar body ultrastructure observed between mutant and corrected cells, despite the clumped growth pattern of mutant iAEC2s. The altered morphological appearance of mutant iAEC2s included a lack of the characteristic apical-facing "bucket"-like shape of EPCAM immunostaining observed in corrected iAEC2s (Figure 3B), suggesting potential apical-basal polarity defects in mutant cells. To directly assess polarity we immunostained for the apical epithelial markers MUC1, phosphorylated ezrin/radixin/moesin (p-ERM), and CRB3 and the adherens junction protein E-cadherin/CDH1 (Figure S2B). We found that while in corrected iAEC2s MUC1, p-ERM, and CRB3 localized to the luminal apical cell surface and E-cadherin localized on the basal-lateral membrane, mutant iAEC2s exhibited diffuse distribution of these markers across all surfaces (Figure S2B), indicating disrupted apical-basal polarity.

To examine the effects of the SFTPC ${ }^{173 T}$ mutation on human surfactant protein processing, we analyzed protein extracts from normal primary adult AEC2s in comparison to corrected versus mutant iAEC2s. In humans, SFTPC protein is produced

(E) Histograms show higher proliferation rates in SFTPC ${ }^{\text {tdT/WT }}$ compared to SFTPC ${ }^{173 T / t d T}$ iAEC2s, measured by flow cytometry as the frequency of SFTPC ${ }^{\text {tdTomato+ }}$ cells that incorporate EdU.

(F) Representative confocal immunofluorescence microscopy of SFTPC ${ }^{\text {tdT/WT }}$ and SFTPC ${ }^{173 T / t d T}$ iAEC2s stained for activated caspase 3 (green) and DNA (Hoechst, blue) shows absence of significant apoptosis. Scale bars, $50 \mu \mathrm{m}$.

( $A$ and $C-E$ ) Mean $\pm S D$ is shown; $n=3$ experimental replicates of independent wells of a differentiation. 
A
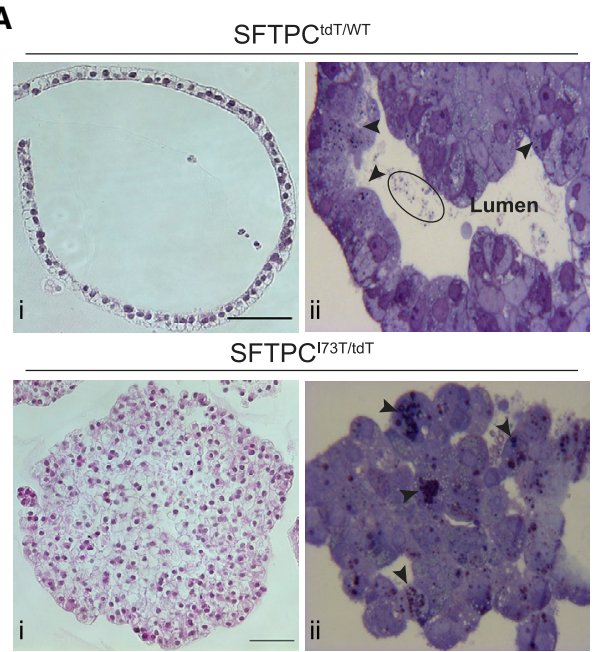

D

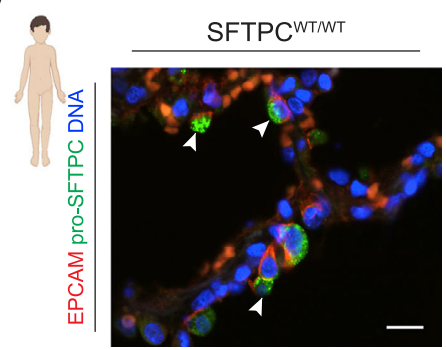

F

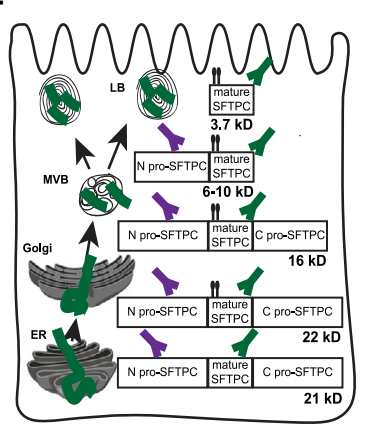

G
B
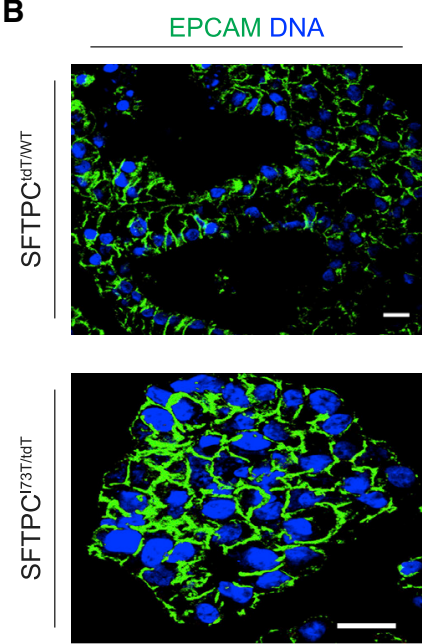

E

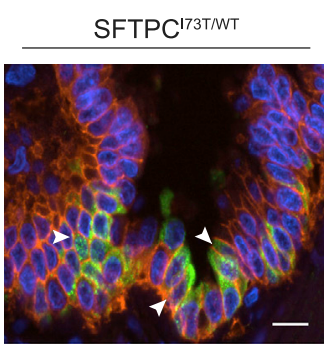

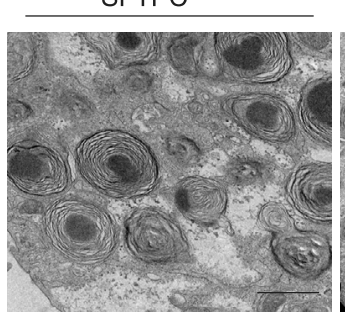

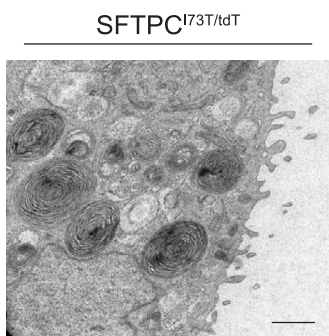

H

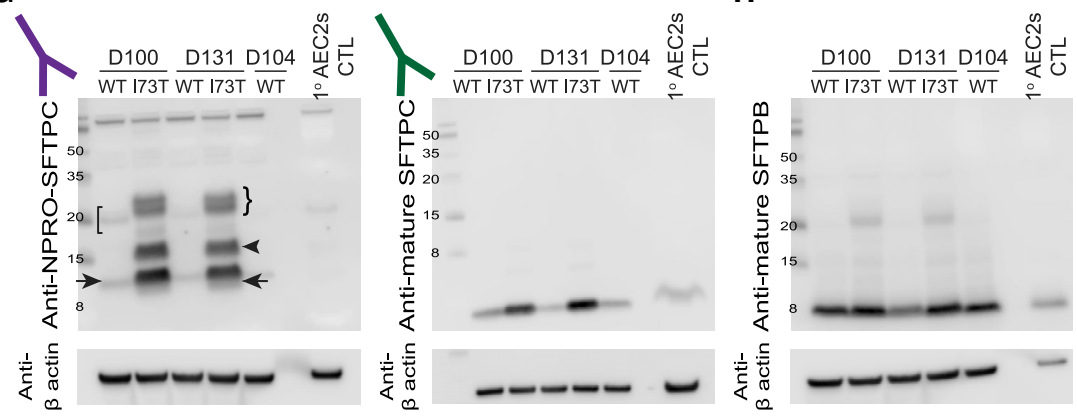

I
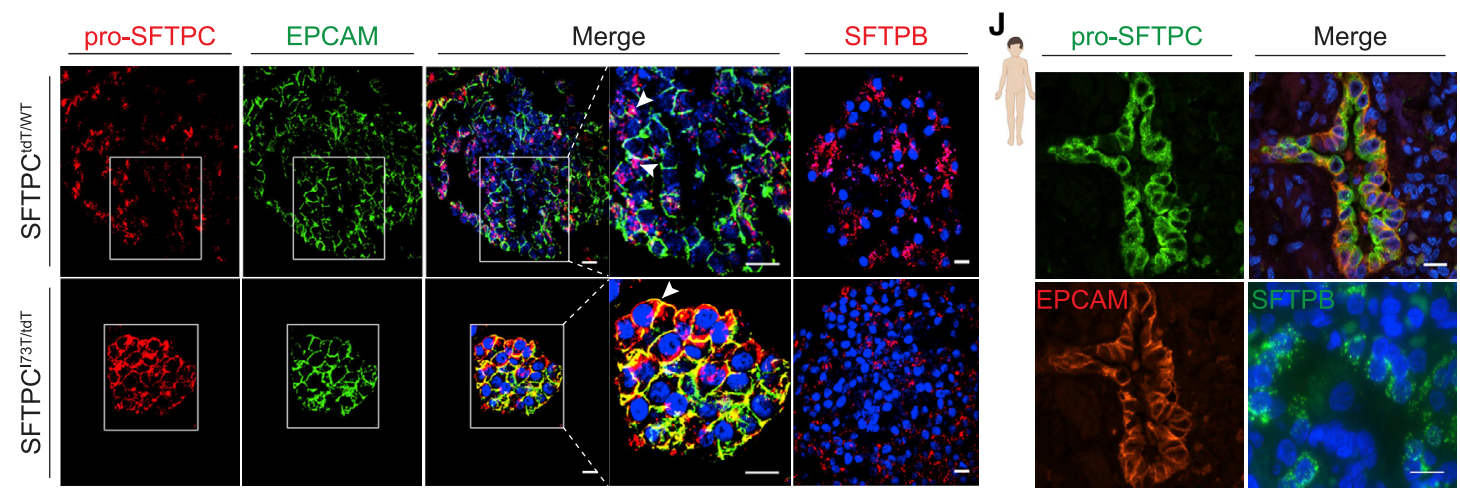

(legend on next page) 
exclusively by $\mathrm{AEC} 2 \mathrm{~s}$ as a $21 \mathrm{kDa}$ pro-protein that undergoes four endoproteolytic cleavages to generate the mature $3.7 \mathrm{kDa}$ form for secretion (Figure 3F) (Beers et al., 1994; Beers and Mulugeta, 2005; Brasch et al., 2002). Complete biosynthesis requires directed anterograde trafficking from Golgi to lamellar bodies, and only cells expressing these organelles are capable of fully processing SFTPC to its mature form. Western blot analysis revealed that mutant iAEC2s accumulated both significant amounts of misprocessed pro-SFTPC forms (Figure 3G, arrowhead) as well as increased amounts of incompletely processed intermediate forms (Figure 3G, right arrow), suggesting aberrant post-Golgi targeting coupled with inefficient posttranslational processing of the SFTPC ${ }^{173 T}$ primary translation product and intermediates (Figure 3G, right bracket and arrow, respectively). In contrast, corrected $\mathrm{iAEC} 2 \mathrm{~s}$ demonstrated restoration of a normal pro-SFTPC processing pattern (Figure 3G, left bracket and arrow) and production of mature $3.7 \mathrm{kDa}$ SFTPC (Figure 3G, right panel). Despite inefficient turnover of mutant proSFTPC $^{173 T}$, production of mature $3.7 \mathrm{kDa}$ SFTPC was also observed in mutant iAEC2s but accumulated to a greater degree than in corrected iAEC2s, suggesting its turnover is also inefficient (Figure 3G, right panel). By comparison, mutant iAEC2s demonstrated intact SFTPB protein processing generating roughly similar amounts of mature $8 \mathrm{kDa}$ SFTPB (Figure $3 \mathrm{H}$ ), suggesting that differences in mutant SFTPC posttranslational processing and turnover were likely not a result of a broader AEC2 trafficking dysfunction. In support of this, immunostaining revealed normal pro-SFTPC intracellular localization to lamellar bodies of corrected iAEC2s, observed as distinct cytoplasmic puncta (Figure 3l, arrowheads), whereas in mutant iAEC2s, pro-SFTPC was mislocalized to the plasma membrane (Figure 3I, arrowhead). Mistrafficking of pro-SFTPC to the plasma membrane was also demonstrated in vivo in sections of the SPC2 donor lung explant (Figures $3 \mathrm{D}$ and $3 \mathrm{~J}$ ). Similar to the normal SFTPB processing profile, immunostaining revealed normal intracellular localization of SFTPB in both mutant and corrected iAEC2s in vitro (Figure 3I) as well as in the donor patient's lung tissue in vivo (Figure $3 \mathrm{~J}$ ). Misprocessing and mistrafficking of pro-SFTPC was also validated in the SPC2 line biallelically expressing both the mutant and "footprint-free" corrected SFTPC alleles (SFTPC ${ }^{173 T / W T}$ ) (Figures S1C and S1D) as well as the SPC6 and SPC7 iPSC lines (Figure S1E).

Transcriptomic and proteomic profiling of SFTPC ${ }^{173 T}$ iAEC2s and their gene-corrected counterparts identifies differential regulation of the lysosomal/ autophagy pathway

We next sought to identify the downstream consequences of pro-SFTPC misprocessing and mistrafficking by profiling mutant and corrected iAEC2s by single-cell RNA sequencing (scRNAseq), bulk RNA sequencing, and proteomic analyses. Transcriptomes profiled at single-cell resolution were more similar at early time points (day 30) after the initial onset of SFTPC expression but diverged over time at later time points (day 113 or day 114) in two repeated experiments (Figures 4A and S3A; day 114 scRNA-seq experiment includes all live cells irrespective of SFTPC ${ }^{\text {tdTomato }}$ expression). For example, only 224 genes were differentially expressed at day 30 , whereas 1,107 genes were differentially expressed by day 113 between mutant and corrected iAEC2s (false discovery rate $[F D R]<0.05$ ) (Table S2). Cell populations at each time point remained similarly pure in terms of frequencies of expression of NKX2-1, SFTPB (Figures 4B, S3B, and S3C), SFTPC (Figures 4C, 4D, S3B, and S3C), as well as the AEC2 program (Figures 4D, S3B, and S3C), based on quantification of expression of our previously defined 8-gene AEC2 differentiation and 5-gene AEC2 maturation benchmarks established using primary adult AEC2s (Hurley et al., 2020). Importantly, the cells did not detectably assume alternative lung or non-lung fates based on little to no expression of airway (SCGB1A1, FOXJ1, TP63, and KRT5) (Figure 4B) (Hurley et al., 2020), endothelial (CDH5) (Figure 4B), hematopoietic $(P T P R C)$ (Figure 4B), or non-lung endodermal (CDX2, AFP, and $A L B$ ) (Figure $\mathrm{S} 3 \mathrm{C}$ ) transcripts.

Although both mutant and corrected iAEC2s captured for scRNA-seq analysis expressed similar frequencies of the AEC2 program (Figure S3C), mutant cells exhibited significantly higher expression levels of AEC2 marker genes (Figures 4B-4E) and less proliferation (significantly lower MKI67 expression and lower frequencies of cells in G2/S/M cell-cycle phases) (Figures 4B, $4 \mathrm{D}$, and S3B). For example, 11 out of the top 50 differentially

Figure 3. SFTPC ${ }^{173 T / \text { tdT }}$ iAEC2s demonstrate distinct cellular morphology and misprocess and mistraffick pro-SFTPC similarly to in vivo SFTPC ${ }^{173 T}$-expressing AEC2s

(A) Representative H\&E staining of formalin fixed and paraffin embedded sections (i, scale bars, $50 \mu \mathrm{m}$ ) and toluidine blue staining of plastic sections (ii) of SFTPC $^{\text {tdT/NT }}$ and SFTPC ${ }^{173 T / d T T}$ iAEC2s. Arrowheads indicate lamellar body-like inclusions; eclipse indicates intraluminal inclusions.

(B) Representative confocal immunofluorescence microscopy of SFTPC ${ }^{\text {tdT/WT }}$ and SFTPC ${ }^{173 T / \mathrm{dT} T}$ iAEC2s for EPCAM (green) and DNA (Hoechst, blue). Scale bars, $10 \mu \mathrm{m}$.

(C) Representative staining of E18.5 $\mathrm{C}^{\mathrm{FlP}}$-SFTPC ${ }^{173 \mathrm{~T} / 73 \mathrm{~T}}$ and $\mathrm{C}^{\mathrm{Flp}}$-SFTPC ${ }^{\mathrm{WT} / \mathrm{WT}}$ embryos for HA-tagged SFTPC. Scale bars, $70 \mu \mathrm{m}$.

(D) Representative confocal immunofluorescence microscopy of distal sections of SPC2 donor and healthy donor lung explants stained for EPCAM (red), proSFTPC (green), and DNA (Hoechst, blue). Scale bars, $10 \mu \mathrm{m}$.

(E) Representative TEM images of SFTPC ${ }^{\text {tdT/WT }}$ and SFTPC ${ }^{173 T / \mathrm{tdT}}$ iAEC2s depicting lamellar bodies. Scale bars, $1 \mu \mathrm{m}$.

(F) Schematic representing the cellular compartments in which pro-SFTPC processing into mature SFTPC occurs. ER, endoplasmic reticulum; MVB, multivesicular body; LB, lamellar body.

$(G$ and $H)$ Representative western blots of SFTPC ${ }^{\text {tdT/WT }}$ and SFTPC ${ }^{173 T / t d T}$ iAEC2 lysates at the indicated time points were compared to freshly isolated primary human AEC2s lysates for pro-SFTPC (NPRO-SFTPC) (G, left panel), mature SFTPC (G, right panel), and mature SFTPB (H) with $\beta$ actin as a loading control.

(I) Representative confocal immunofluorescence microscopy of SFTPC ${ }^{\text {tdT/WT }}$ and SFTPC ${ }^{173 T / d T}$ iAEC2s for pro-SFTPC (red; with zoom), EPCAM (green; with zoom), SFTPB (red), and DNA (Hoechst, blue). Scale bars, $10 \mu \mathrm{m}$.

(J) Representative confocal immunofluorescence microscopy of distal sections of SPC2 donor and healthy donor lung explants for pro-SFTPC (green), EPCAM (red), SFTPB (green), and DNA (Hoechst, blue). Scale bars, $10 \mu \mathrm{m}$. 
A

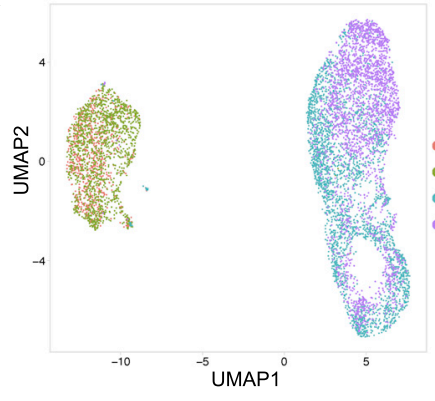

C

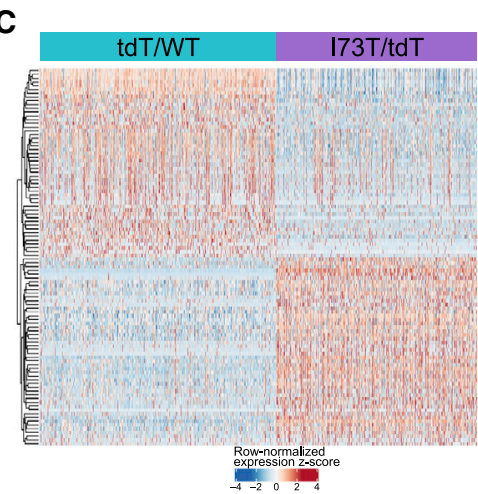

F

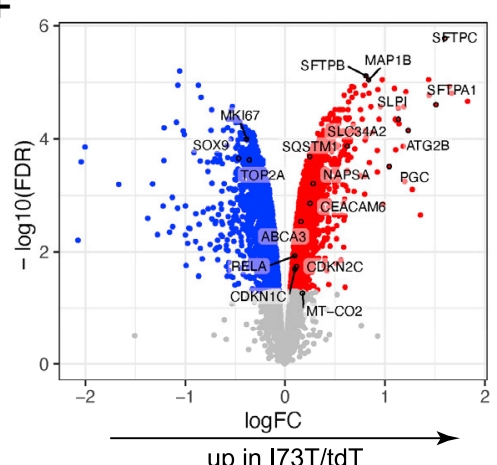

H

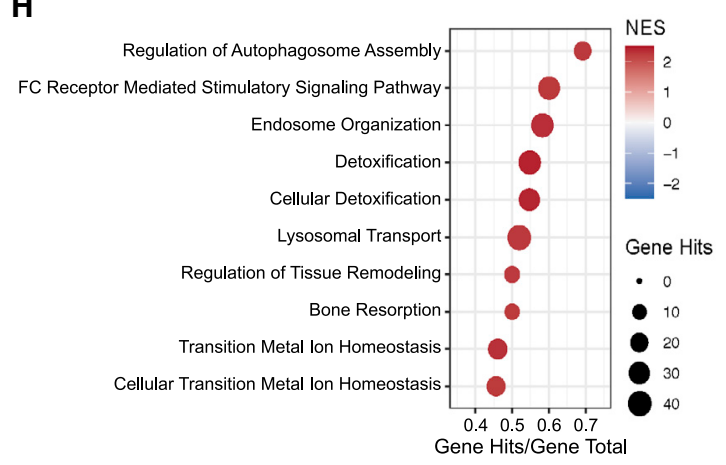

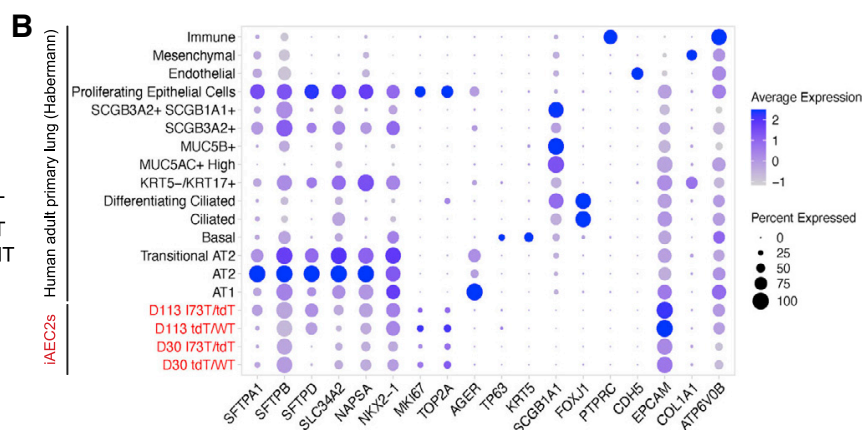

D

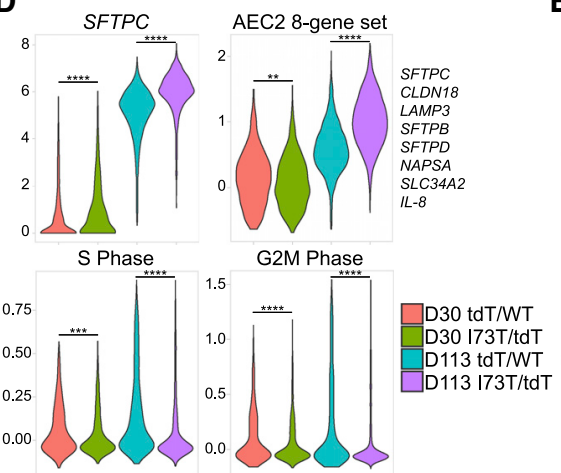

E

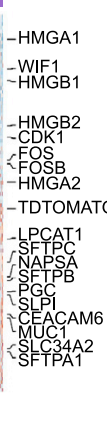

G

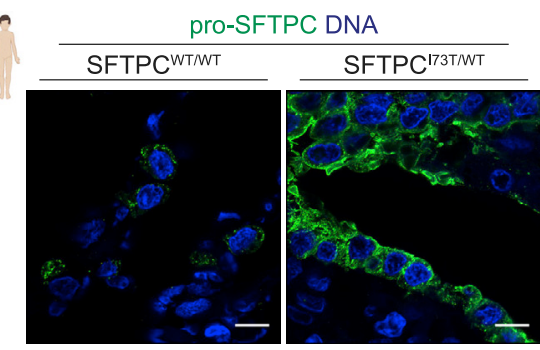

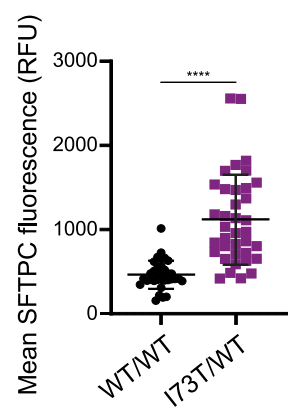

I NES Gene Ratio

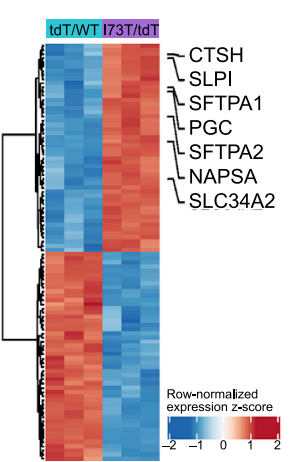

tdT/WT 173T/dT

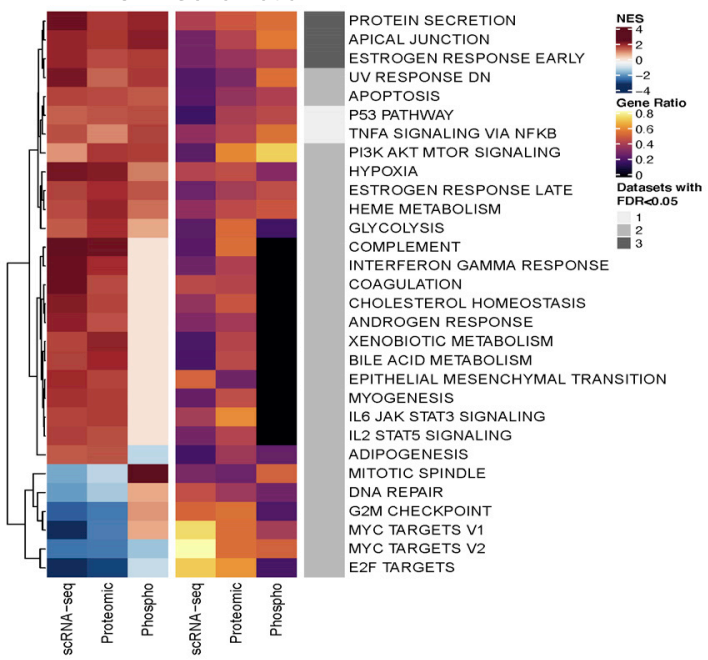

Figure 4. Transcriptomic and proteomic/phosphoproteomic analyses identify candidate disease-associated pathways

(A) Clustering of SFTPC ${ }^{\text {tdTNTT }}$ and SFTPC ${ }^{173 T / \text { tdT }}$ iAEC2 scRNA-seq transcriptomes at two time points (days (d)30 and 113 of differentiation) using Uniform Manifold Approximation Projection (UMAP). 
upregulated genes in mutant iAEC2s in the day 113 scRNA-seq were composed of transcripts encoding surfactants, lamellar body-related, and other AEC2-marker genes (PGC, SFTPB, SLPI, NAPSA, SLC34A2, LPCAT1, SFTPA1, CEACAM6, MUC1, SFTPC, and TDTOMATO) (Figure 4C). We validated these findings by real-time qPCR quantitation (Figure S3D) as well as in independent experiments analyzed by bulk RNA-seq at 2 time points, day 114 and day 131 of differentiation, again finding AEC2 gene markers comprised $14 \%$ of the top 50 most upregulated genes in mutant iAEC2s (Figure 4E).

By global proteomic analysis performed on the same day as scRNA-seq, SFTPC was the most enriched protein overall in mutant versus corrected iAEC2s (ranked by FDR) (Figure 4F), consistent with our SFTPC western blot and immunostaining findings. Additional AEC2 proteins were also over-represented in the top 50 most upregulated proteins in mutant cells, including SFTPB, SFTPA1, SLPI, and PGC, suggesting that both transcripts and proteins encoding multiple surfactants and other AEC2 markers were in the top most upregulated in mutant iAEC2s. Proteomic analysis also validated the less proliferative state of mutant iAEC2s as MKI67 protein was enriched in corrected cells, whereas cell-cycle inhibitors CDKN1C and CDKN2C were upregulated in mutant cells (Figure 4F). Analysis of SPC2 donor's lung explant sections also revealed a higher in vivo pro-SFTPC immunostaining intensity compared to control distal lung (Figure 4G), suggesting accumulation of mutant proSFTPC in the patient's AEC2s.

Having established that mutant iAEC2s display a less proliferative and more mature AEC2 phenotype than corrected cells with marked accumulation of SFTPC protein, we next sought to determine signaling pathways or biological processes that might differ in mutant cells. The autophagy-related protein ATG2B, essential for lysosomal formation, was in the top 10 most upregulated proteins (ranked by logFC) in mutant cells (Figure $4 \mathrm{~F}$ ) and gene set analysis of GO, KEGG, and HALLMARK terms using both proteomic, scRNA-seq, and bulk RNA-seq analyses all revealed significant upregulation of lysosomal and autophagy-related terms in mutant cells (Figures $4 \mathrm{H}$ and S3E). A systems-based approach integrating transcriptomic, proteomic, and phosphoproteomic analyses revealed a high degree of concordant regulation with protein secretion processes being perturbed across all datasets consistent with the build-up of secretory proteins evident on western blots and immunostains. In addition, altered apical junctional processes, glycolysis, STAT3 signaling, MTOR signaling, and inflammatory signaling via nuclear factor $\kappa \mathrm{B}(\mathrm{NF}-\kappa \mathrm{B})$ implied additional broad perturbations were present across a wide variety of epithelial, metabolic, and inflammatory cellular processes in mutant iAEC2s, whereas proliferation pathways (E2F and MYC targets) were downregulated (Figure 4I). Given that mutant and corrected iAEC2s are (with the exemption of the SFTPC ${ }^{173 T}$ mutation) otherwise genetically identical, the data above suggest that expression of mutant SFTPC drives a transcriptomic program that markedly alters the IAEC2 phenotype and includes alterations in protein expression, quality control, and intracellular signaling.

\section{SFTPC ${ }^{173 T}$ iAEC2s display autophagy perturbations} Given the potential effect of the SFTPC ${ }^{173 T}$ mutation on autophagy suggested by our bioinformatic analyses (Figure $4 \mathrm{H}, \mathrm{I}$ ), we employed a variety of independent static and dynamic approaches to evaluate the autophagy pathway in iAEC2s (Figure 5A). We first assessed the intracellular levels of microtubule-associated protein 1 light chain 3 (LC3), a marker of autophagosome formation (Klionsky et al., 2016). Western blot analysis showed elevated LC3, suggesting an increased autophagosomal mass in mutant compared to corrected iAEC2s (Figure 5B). To distinguish between enhanced autophagosome formation versus reduced degradation, we examined the levels of p62 (SQSTM1). P62 through direct interaction with LC3, facilitates transfer of polyubiquitinated proteins to the completed autophagosome where it is degraded along with its cargo, thus serving as a marker of autophagic degradation. Mutant iAEC2s demonstrated significant accumulation of p62, compared to corrected $\mathrm{i} A E C 2 \mathrm{~s}$, consistent with a reduction in autophagosome degradation (Figure 5B). To further delineate if the accumulation of LC3 in mutant iAEC2s was the result of only reduced autophagosome degradation or a concomitant induction in autophagosome formation, we performed autophagic flux studies. We first examined the response to the mTOR inhibitor/autophagy activator torin. When compared to their corrected counterparts, mutant iAEC2s demonstrated significantly lower LC3 turnover following treatment with torin for $18 \mathrm{~h}(55.2 \% \pm 9.4 \%$ versus

\footnotetext{
(B) Average expression levels and frequencies (purple dots) for select genes profiled by scRNA-seq in SFTPC $C^{\text {tdT/NT }}$ and SFTPC ${ }^{173 T / t d T}$ iAEC2s. Comparison is made to a publicly available adult primary distal lung dataset (Habermann et al., 2020), and genes are selected to indicate AEC2, AEC1, airway, endothelial, epithelial, leukocyte, or proliferation programs.

(C) Heatmap of top 50 upregulated and top 50 downregulated genes comparing d113 SFTPC ${ }^{\text {tdT/WT }}$ versus d113 SFTPC ${ }^{173 T / \text { tdT }}$ iAEC2s by scRNA-seq (ranked by average log fold-change, FDR $<0.05$; row-normalized expression $Z$ scores). A subset of differentially expressed genes is highlighted with large font.

(D) Violin plots showing normalized expression for indicated genes or cell cycle phase in d30 and d113 SFTPC ${ }^{\text {tdT/WT }}$ versus SFTPC ${ }^{173 T / t d T}$ iAEC2s by scRNA-seq. (E) Heatmap of the 50 upregulated and top 50 downregulated genes in d114 SFTPC ${ }^{\text {tdT/WT }}$ versus d114 SFTPC ${ }^{173 T / t d T}$ iAEC2s by bulk RNA seq (ranked by FDR, FDR $<0.05$; row-normalized expression $Z$ scores). AEC2-marker genes are highlighted with large font.

(F) Volcano plots indicating differential protein expression in d113 SFTPC ${ }^{\text {tdT/WT }}$ versus d113 SFTPC ${ }^{173 T / \text { tdT }}$ iAEC2s by mass spectrometry.

(G) Representative confocal immunofluorescence microscopy of distal sections of SPC2 donor and healthy donor lung explants stained for pro-SFTPC (green) and DNA (Hoechst, blue) shows an altered cellular localization pattern and a higher pro-SFTPC fluorescence intensity in the patient's AEC2s, quantified by relative fluorescence units (RFU). Scale bars, $10 \mu \mathrm{m}$ (mean \pm SD; $\mathrm{n}=35$ individual SFTPC-positive cells treated as experimental replicates). (H) Top 10 upregulated pathways in d113 SFTPC ${ }^{173 T / \text { td T }}$ versus d113 SFTPC ${ }^{\text {tdT/WT }}$ iAEC2s based on global proteomic analysis.

(I) Integrated gene set enrichment analyses based on scRNA-seq transcriptomic, proteomic, and phosphoproteomic analyses in $\mathrm{d} 113 \mathrm{SFTPC}{ }^{173 T / t d \mathrm{TT}}$ versus $\mathrm{d} 113$ SFTPC ${ }^{\text {tdT }}$ TT $i$ AEC2s with an FDR $<0.1$ in at least 2 out of 3 datasets. NES, normalized enrichment score; gene ratio, ratio of enriched genes for a given pathway to the total number of genes in the pathway. The last column represents the number of datasets (out of 3) with an FDR $<0.05$.

${ }^{\star \star} \mathrm{p}<0.01,{ }^{\star \star \star} \mathrm{p}<0.001$, and ${ }^{\star \star \star \star} \mathrm{p}<0.0001$ by unpaired, two-tailed Student's $\mathrm{t}$ test for all panels.
} 
A

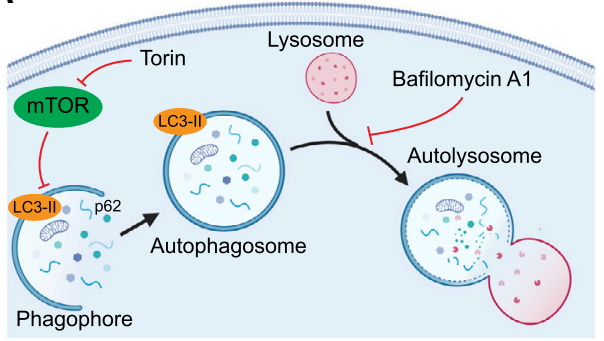

B

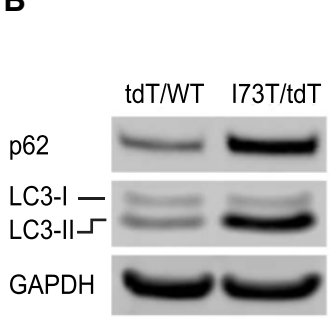

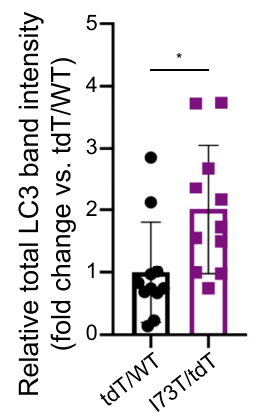

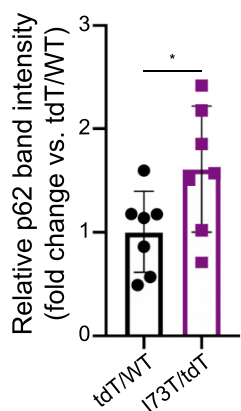

C $\frac{\text { Vehicle Torin } 5 \mu \mathrm{M}}{4 \mathrm{~h} 18 \mathrm{~h}} \frac{\mathrm{BafA} 1}{4 \mathrm{~h} 8 \mathrm{~h} 18 \mathrm{~h}} \frac{\dot{\mathrm{V}} \text { Vehicle Torin } 5 \mu \mathrm{M} \text { BafA1 }}{50100} \frac{\mathrm{E}}{4 \mathrm{~h} 18 \mathrm{~h}}$

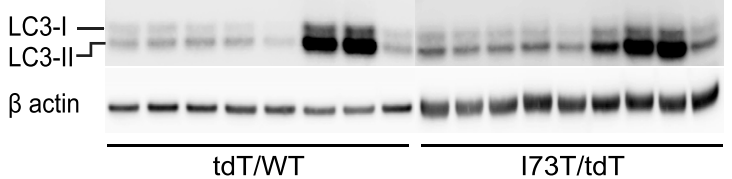

D

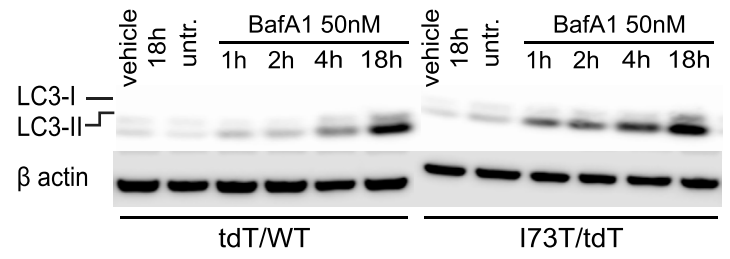

E
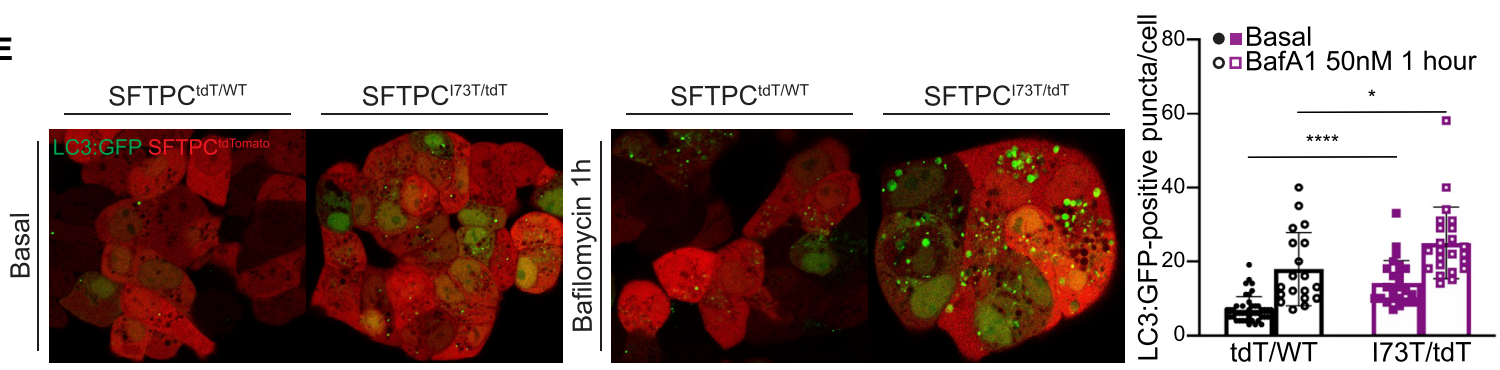

$\mathbf{F}$

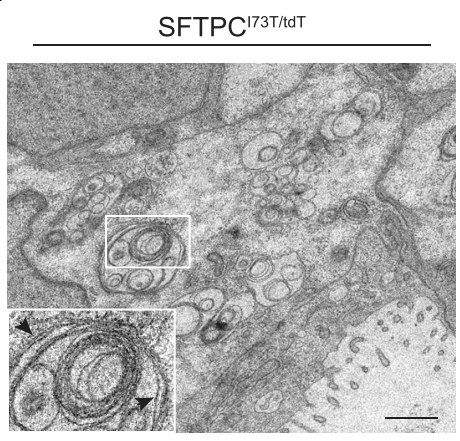

G

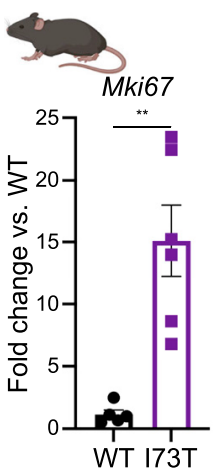

H
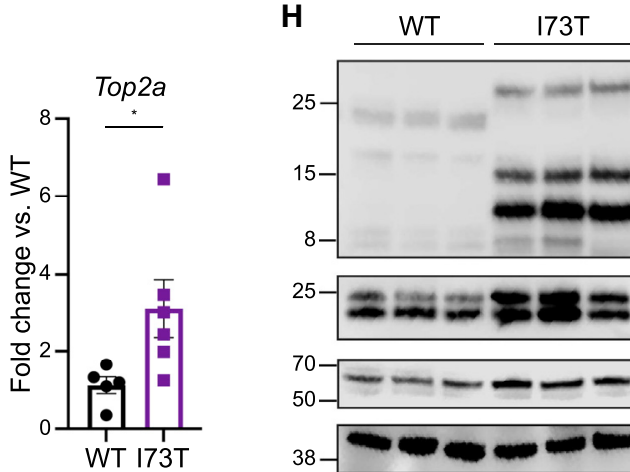

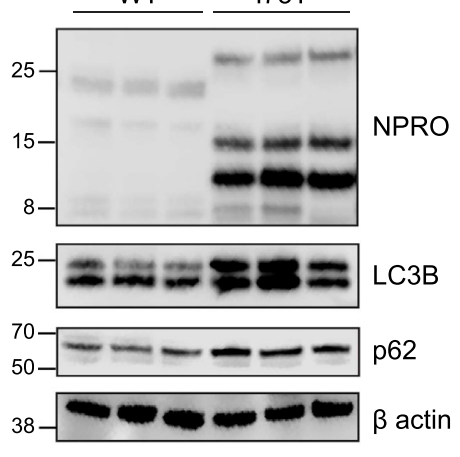

K
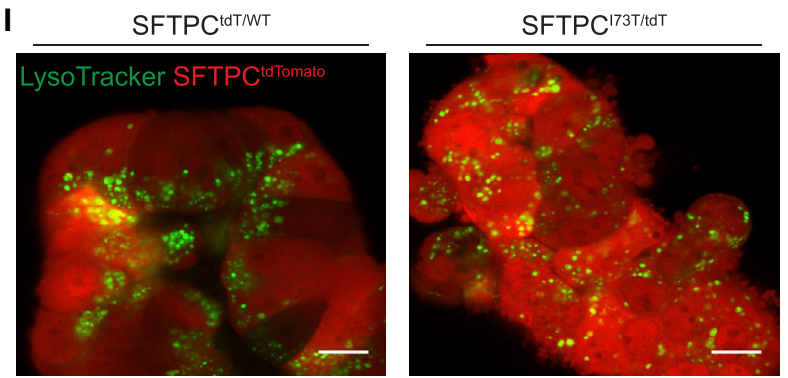

$\mathbf{J}$
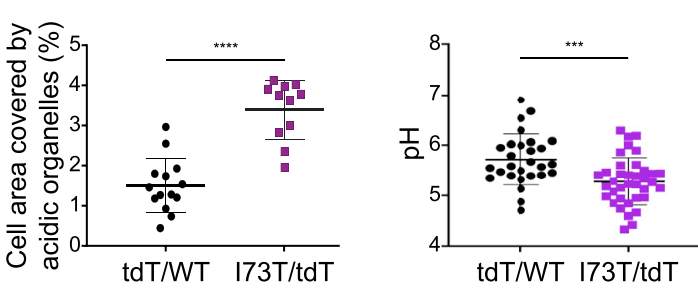

Figure 5. Autophagy perturbations in SFTPC ${ }^{173 T}$ iAEC2s

(A) Schematic illustrating the autophagy pathway. Key proteins involved in the pathway (p62/SQSTM1 and LC3) and the mechanisms of action of bafilomycin A1 and torin are depicted. 
$26.7 \% \pm 0.3 \%$ reduction over baseline, $p=0.05$ ) (Figure $5 \mathrm{C}$ ). We then examined the effect of bafilomycin A1 (BafA1), a vacuolartype $\mathrm{H}^{+}$-ATPase (V-ATPase) inhibitor, which inhibits autophagosome degradation via both neutralizing the lysosomal $\mathrm{pH}$ and by blocking the fusion of autophagosomes with lysosomes (Klionsky et al., 2016; Yamamoto et al., 1998). Treatment with BafA1 $50 \mathrm{nM}$ resulted in earlier accumulation and higher amounts of LC3 in mutant compared to corrected iAEC2s suggesting increased autophagosome formation (Figure 5D). The autophagy perturbations observed in mutant (SFTPC ${ }^{173 T / t d T}$ ) iAEC2s were further validated in the SPC2 line biallelically expressing both mutant and corrected SFTPC alleles (SFTPC ${ }^{173 T / W T}$ ), as evidenced by the accumulation of both LC3 and p62 in mutant iAEC2s (Figure S1C). Together, these results suggest a concomitant upregulation of autophagosome formation (flux) in mutant iAEC2s as well as a late block in autophagy (autophagosome turnover) consistent with our previous studies in heterologous cell lines stably expressing SFTPC ${ }^{173 T}$ and in vivo in our Sftpc ${ }^{173 T}$ mouse model (Hawkins et al., 2015; Nureki et al., 2018).

To further corroborate the observed changes in biochemical markers of autophagy, we next utilized advanced imaging techniques to assess autophagosome dynamics in individual iAEC2s. We first transduced mutant and corrected iAEC2s with a lentiviral construct constitutively expressing GFP fused to LC3 (LC3:GFP) (Twig et al., 2008) to visualize and quantitate autophagosome numbers, which are represented by cytosolic GFP-positive punctae. Consistent with the biochemical profile (Figure 5B), under steady-state conditions, we found more autophagosomes in mutant versus corrected iAEC2s (Figure 5E). The fusion between autophagosomes and lysosomes promotes the degradation of both LC3 and GFP facilitated by low autophagolysosome $\mathrm{pH}$ that can be blocked by treatment with BafA1 ( $\mathrm{Ni}$ et al., 2011). Treatment with BafA1 $50 \mathrm{nM}$ for $1 \mathrm{~h}$ enhanced both GFP fluorescence and the numbers of GFP-positive punctae in mutant iAEC2s compared to similarly treated corrected iAEC2s consistent with increased autophagic flux (Figure 5E). Similar observations were made in the SPC6 iPSC line where increased numbers of GFP-positive punctae were identified at both steady-state conditions and following treatment with
BafA1 (50 nM for $1 \mathrm{~h}$ ), compared to corrected (SFTPC ${ }^{t d T / W T}$ ) iAEC2s (data not shown). Morphologically, the SPC6 iAEC2s lacked lumens and formed ball-like structures (data not shown) similar to SFTPC ${ }^{173 T / t d T}$ iAEC2s (Figures $3 \mathrm{~A}$ and 3B). The changes in autophagosome dynamics in mutant iAEC2s were further confirmed using ultrastructural analysis by TEM where double membrane intracellular vacuoles characteristic of autophagosomes were easily visualized in mutant iAEC2s, whereas these structures remained sparse in any of the corrected iAEC2s (Figure 5F).

We further compared our AAEC2 in vitro model to in vivo changes that arise soon after induction of the Sftpc mutation in tamoxifen-inducible Sftpc ${ }^{173 T}$ knockin mice. Fourteen days after in vivo administration of tamoxifen or vehicle to adult mice, we isolated $\mathrm{AEC} 2 \mathrm{~s}$ to profile the proliferation state and autophagy pathway. In contrast to iAEC2s after prolonged culture periods, mouse mutant AEC2s were more proliferative compared to wild-type (WT) AEC2s (Figure 5G), possibly attributed to the in vivo setting with alternative lineages present. Nonetheless, mouse mutant AEC2s demonstrated similar pro-SFTPC misprocessing and autophagy perturbations with accumulation of both LC3 and p62 (Figure 5H), suggesting that the differences in proliferation states between mutant and corrected AEC2s did not account for the observed autophagic changes.

Because fusion with acidic lysosomes is required for autophagosome degradation, we considered the possibility that failure of lysosomes to properly acidify might explain the block in autophagy observed in mutant iAEC2s. We therefore stained mutant and corrected iAEC2s with LysoTracker green (a basic dye that stains acidic vesicular organelles) (Figure $5 \mathrm{I}$ ) and found that mutant iAEC2s contained more acidic organelles compared to corrected cells (Figure 5J). To better assess the iAEC2 lysosomal $\mathrm{pH}$, we used Lysosensor yellow/blue-dextran (Figure S4), a pHsensitive fluorescent probe targeting the lysosomes with high specificity (Wolfe et al., 2013). Quantitation of probe fluorescence revealed that lysosomes of mutant iAEC2s maintained a lower intraluminal $\mathrm{pH}$ when compared to those of corrected iAEC2s (Figure 5K). Together, these data indicate that mutant iAEC2s exhibit an accelerated autophagy flux (autophagosome

(B) Representative western blot of SFTPC ${ }^{\text {tdT/WT }}$ and SFTPC ${ }^{173 T / d \mathrm{dT}}$ iAEC2 lysates for p62 and LC3 with GAPDH and $\beta$ actin as loading controls with densitometric quantification (mean $\pm S D ; n=11$ for $L C 3$ and $n=7$ for p62 independent experiments).

(C) Western blots of cell lysates for LC3 and $\beta$ actin from autophagic flux studies using SFTPC ${ }^{\text {tdT } / W T}$ or SFTPC ${ }^{173 T / d \mathrm{dT}}$ iAEC2s treated with either torin (5 $\left.\mu \mathrm{M}\right)$ for 4,8 , or $18 \mathrm{~h}$ or bafilomycin A1 (BafA1) ( 50 or $100 \mathrm{nM}$ ) for $18 \mathrm{~h}$ or vehicle (DMSO) for 4 or $18 \mathrm{~h}$ (blots shown are representative of $\mathrm{n}=2$ independent experiments).

(D) A repeat autophagic flux study, as in (C) but using BafA1 (50 nM) for 1, 2, 4, and $18 \mathrm{~h}$ or vehicle (DMSO) for $18 \mathrm{~h}$ (representative of $\mathrm{n}=2$ independent experiments).

(E) SFTPC ${ }^{\text {tdT/WT }}$ and SFTPC ${ }^{173 T / t d T}$ iAEC2s transduced with a lentiviral LC3:GFP fusion protein and exposed to BafA1 (50 nM) to quantify autophagosomes and autophagolysosomes. Scale bar, $10 \mu \mathrm{m}$ (representative confocal fluorescence microscopy images of $n=3$ experimental replicates of independent wells of a differentiation).

(F) Representative TEM image of SFTPC ${ }^{173 T / d \mathrm{dT}}$ iAEC2s shows a double-membrane autophagosome (inset, arrowheads). Scale bar, $1 \mu \mathrm{m}$.

(G) Real-time qPCR showing fold change in gene expression compared to WT in primary mouse Sftpc ${ }^{173 T} \mathrm{AEC} 2 \mathrm{~s}$ ( mean $\pm \mathrm{SEM} ; \mathrm{n}=6$ for Sftpc ${ }^{173 T}$ and $\mathrm{n}=5$ for WT mice).

(H) Representative western blots of primary mouse WT and Sftp ${ }^{173 T}$ AEC2 lysates for pro-SFTPC (NPRO), LC3, and p62 with $\beta$ actin as a loading control (see also Figure 3G) ( $\mathrm{n}=3 \mathrm{WT}$ and $\mathrm{Sftpc}^{173 T}$ mice).

(I) Representative live-cell confocal fluorescence microscopy of SFTPC ${ }^{\text {tdT/WT }}$ and SFTPC ${ }^{173 T / t d T}$ iAEC2s stained with LysoTracker green. Scale bars, $10 \mu \mathrm{m}$. (J) Quantification of acidic organelles expressed as percentage of covered cell area (mean \pm SD; $n=11-14$ independent alveolospheres treated as experimental replicates)

(K) Quantification of lysosomal pH based on Lysosensor yellow/blue-dextran ratio (mean $\pm \mathrm{SD} ; \mathrm{n}=2$ independent experiments with 20-30 cells analyzed per experiment; see also Figure S4).

${ }^{*} \mathrm{p}<0.05,{ }^{* \star} \mathrm{p}<0.01,{ }^{* \star} \mathrm{p}<0.001$, and ${ }^{\star \star * *} \mathrm{p}<0.0001$, by unpaired, two-tailed Student's $\mathrm{t}$ test for all panels. 
A

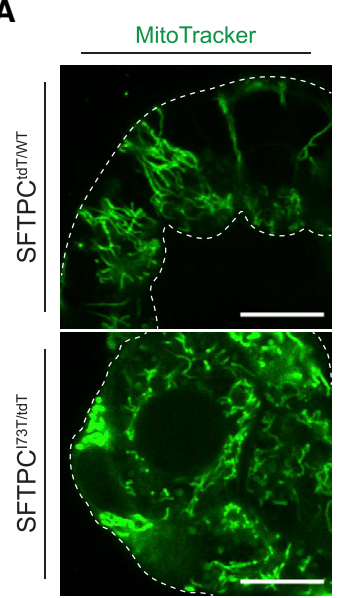

$\mathbf{F}$

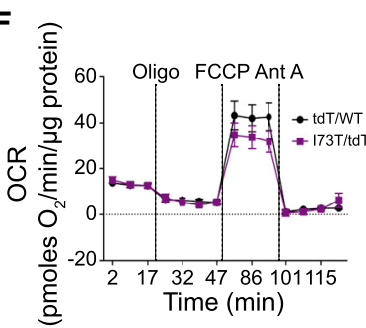

B

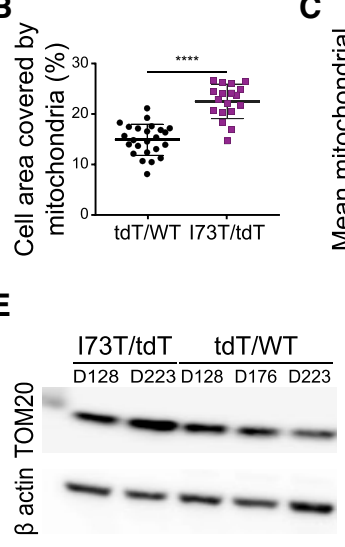

$\mathbf{H}$

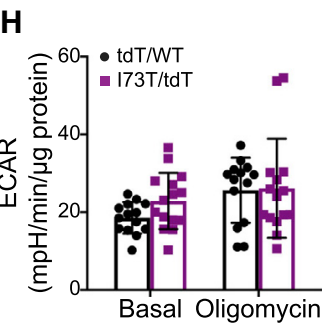

C
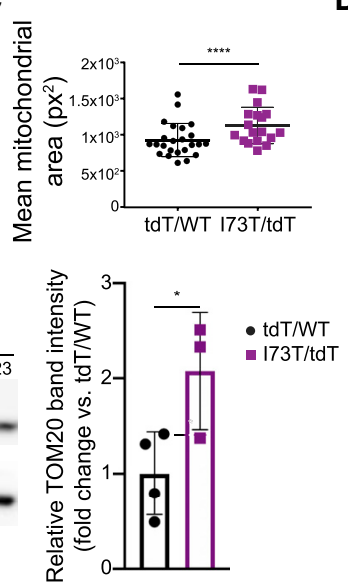

D

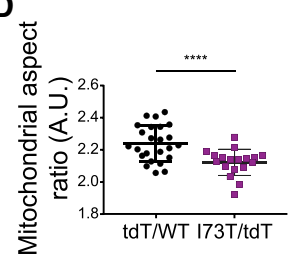

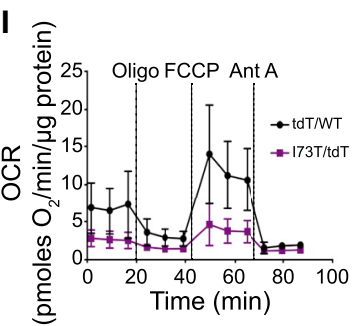

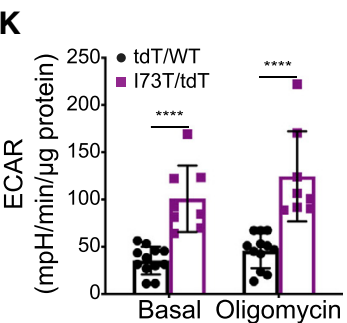

G Proton Leak $\mathbf{J}$

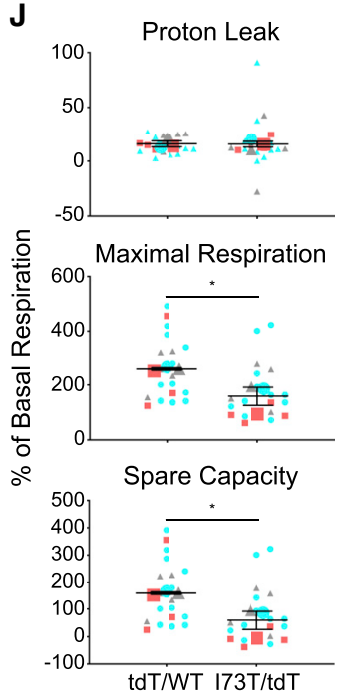

$\mathbf{L}$
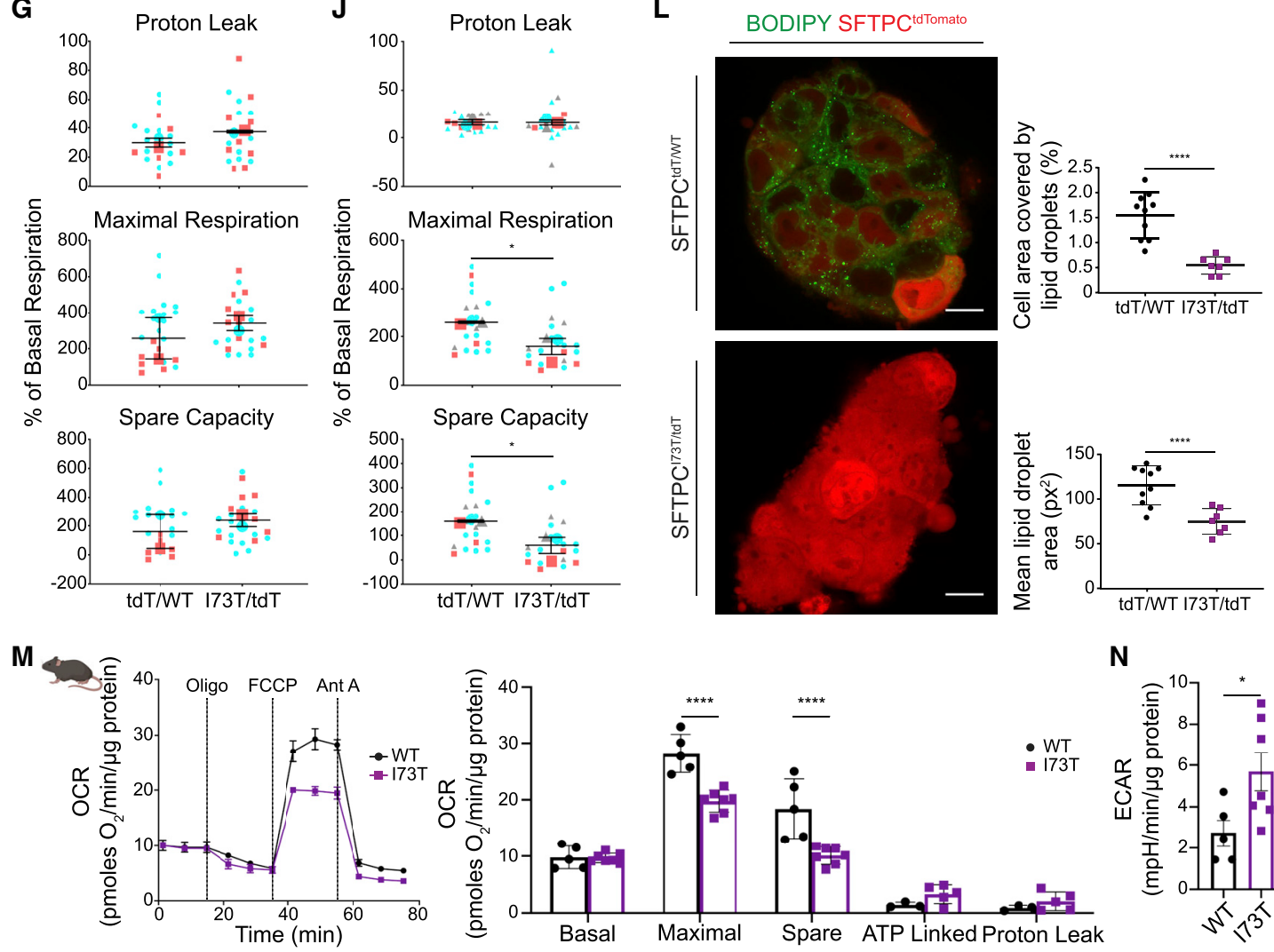

N

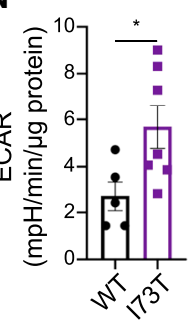

Figure 6. SFTPC ${ }^{173 T}$-expressing AEC2s demonstrate metabolic reprogramming

(A) Representative live-cell confocal fluorescence microscopy of SFTPC ${ }^{\text {tdT/WT }}$ and SFTPC ${ }^{173 T / \mathrm{dT} T}$ iAEC2s stained with MitoTracker green. Scale bars, $10 \mu \mathrm{m}$. 
and autophagolysosome formation) without impairment of vacuolar acidification, suggesting that the enhanced production of mutant SFTPC ${ }^{173 T}$ has saturated the autophagic capacity of mutant iAEC2s without altering lysosomal $\mathrm{pH}$.

\section{Mutant human iAEC2s in vitro and mouse AEC2s in vivo demonstrate metabolic reprogramming}

Recognizing that changes in metabolic pathways (i.e., glycolysis) were among the top differentially upregulated pathways in mutant iAEC2s in our integrated analysis (Figure 4I) coupled with our prior work showing that expression of SFTPC ${ }^{173 T}$ could also alter steady-state mitochondrial mass and membrane potential (Hawkins et al., 2015; Nureki et al., 2018), directed us to investigate changes in cellular bioenergetics of mutant iAEC2s. Mitochondrial morphology and mass were assessed using quantitative imaging of MitoTracker dye staining. Distinct mitochondrial morphologies were observed in mutant versus corrected iAEC2s. Although the mitochondria in corrected cells were more elongated forming a network extending from the basal-lateral to the apical cell surface, in mutant cells, mitochondria demonstrated a rounded, fragmented morphology (Figure 6A). Mutant iAEC2s also demonstrated higher mitochondrial mass (Figure $6 \mathrm{~B}$ ) with accumulation of larger (Figure 6C) but more fragmented mitochondria quantified by the aspect ratio (ratio of long over short diameter) (Figure 6D), changes previously described in primary AEC2s from patients with IPF and aged mice (Bueno et al., 2015). The higher mitochondrial mass in mutant iAEC2s was confirmed by the higher levels of mitochondrial protein TOM20, which appeared to be time-dependent with enhanced accumulation as the cells aged in culture (Figure 6E).

To investigate whether the changes in mitochondrial morphology between mutant and corrected iAEC2s were associated with changes in mitochondrial function, we performed respirometry experiments at different time points: earlier time points close to our prior transcriptomic/proteomic analyses (dif- ferentiation days 128 and 153) and later time points (differentiation days 175, 213, and 291). At the earlier time points, there were no significant differences between mutant and corrected iAEC2s in terms of their basal oxygen consumption rate (OCR), maximal uncoupled mitochondrial respiration (assessed after the administration of FCCP), and spare respiratory capacity (Figures 6F and $6 \mathrm{G}$ ). Similar to oxidative phosphorylation, no differences were observed in glycolysis between mutant and corrected iAEC2s, as measured by the extracellular acidification rate (ECAR) either at steady-state or following treatment with the ATP synthase inhibitor oligomycin (Figure 6H). However, at later time points, which correspond to further aged iAEC2s, mutant cells demonstrated a significant reduction in maximal uncoupled mitochondrial respiration and spare respiratory capacity (Figures $6 \mathrm{I}$ and $6 \mathrm{~J}$ ). Conversely, mutant iAEC2s at later time points demonstrated a higher ECAR (Figure 6K) suggestive of a metabolic switch toward glycolytic metabolism. Because mobilization of lipid stores is required not only for energy utilization but also to sustain the expansion of the surface area of the mitochondrial membranes and autophagosomes, we assessed the lipid content of mutant and corrected iAEC2s. We found significantly lower levels of stored triglycerides in mutant iAEC2s, as assessed by BODIPY493/503 dye (Figure 6L).

To provide in vivo validation of our in vitro findings suggesting metabolic reprogramming of mutant iAEC2s from oxidative phosphorylation to glycolysis and to uncouple these changes from the in vitro differences in proliferation rates between mutant and corrected iAEC2s, we performed additional experiments using the same tamoxifen-inducible $S_{\text {ftp }}{ }^{173 T}$ knockin mouse model. AEC2s were isolated 14 days following in vivo tamoxifen or vehicle administration and subjected to respirometry experiments. As shown above (Figure $5 \mathrm{G}$ ), in this in vivo model mutant AEC2s are more proliferative compared to WT cells. Similar to our in vitro model findings, we found a reduction in maximal uncoupled mitochondrial respiration and spare capacity (Figure 6M) as well as increased ECAR (Figure $6 \mathrm{~N}$ ) in the Sftpc ${ }^{173 T}$ mutant

(B-D) Quantitative analyses of morphometric data from fluorescence images (mean \pm SD; $n=24$ SFTPC ${ }^{\text {tdT/WT }}$ and $n=18$ SFTPC ${ }^{\text {I73T/tdT }}$ independent alveolospheres treated as experimental replicates).

(B) Increased mitochondrial mass in SFTPC ${ }^{173 T / t d T}$ iAEC2s, quantified as the percentage of cell area covered by mitochondria.

(C) Increased mitochondrial size in SFTPC ${ }^{173 T / t d T}$ iAEC2s.

(D) SFTPC ${ }^{173 T / t d T}$ iAEC2 mitochondria are more fragmented, assessed by the aspect ratio.

(E) Representative western blot of SFTPC ${ }^{\text {tdT/WT }}$ and SFTPC ${ }^{173 T / t d T}$ iAEC2 lysates for TOM20 with $\beta$ actin as loading control with densitometric quantification (mean $\pm \mathrm{SD} ; \mathrm{n}=4$ [days 128, 176, 223, 223] SFTPC ${ }^{\text {tdT/WT }}$ and $\mathrm{n}=3$ [days 128, 223, 223] SFTPC ${ }^{173 \mathrm{t} / \mathrm{td}}$ independent experiments).

(F) Early time point OCR was measured under basal conditions followed by addition of oligomycin, FCCP, and antimycin A (Ant A), as indicated.

(G) SFTPC ${ }^{\text {tdT/WT }}$ and SFTPC ${ }^{173 T / t d T}$ iAEC2s demonstrate no significant differences in proton leak, maximal respiration, and spare capacity measured as percent change over basal respiration (mean $\pm \mathrm{SEM} ; \mathrm{n}=2$ independent experiments).

$(\mathrm{H})$ Early time point ECAR under basal conditions and following addition of oligomycin.

(I) Late time point OCR.

(J) SFTPC ${ }^{173 T / t d T}$ iAEC2s demonstrate significantly lower maximal respiration and spare respiratory capacity (mean \pm SEM; $n=3$ independent experiments) measured as percent change over basal respiration.

(K) Late time point reveals a significantly higher ECAR in SFTPC ${ }^{173 T / t d T}$ iAEC2s (mean $\pm S D ; n=3$ independent experiments).

(L) Representative live-cell confocal fluorescence microscopy of SFTPC ${ }^{\text {tdT/WT }}$ and SFTPC ${ }^{173 T / t d T}$ iAEC2s stained with BODIPY $493 / 503$ dye with quantitative analyses. Scale bars, $10 \mu \mathrm{m}$ (mean \pm SD; $n=10$ SFTPC $^{\text {tdT/WT }}$ and $n=7$ SFTPC ${ }^{173 T / t d T}$ independent alveolospheres treated as experimental replicates).

(M) OCR measured under basal conditions and following addition of oligomycin, FCCP, and Ant A, as indicated (mean \pm SD; $n=7$ Sftpc $c^{173 T}$ and $n=5$ WT mice). (N) ECAR measured under basal conditions in WT and Sftpc ${ }^{17 T}$ mouse AEC2s (mean \pm SEM; $n=5$ WT mice and $n=7$ Sftpc ${ }^{173 T}$ ). OCR, oxygen consumption rate; ECAR, extracellular acidification rate.

( $G$ and J) Depict super plots: small shapes represent replicate values within each experiment and large shapes represent the average of each independent experiment, tdT/WT and I73T/tdT are color-matched between experiments. ${ }^{*} \mathrm{p}<0.05$ and ${ }^{\star \star \star \star} \mathrm{p}<0.0001$ by unpaired, two-tailed Student's t test for all panels except (M) where a mixed-effects model with Bonferroni's correction was used. 
A

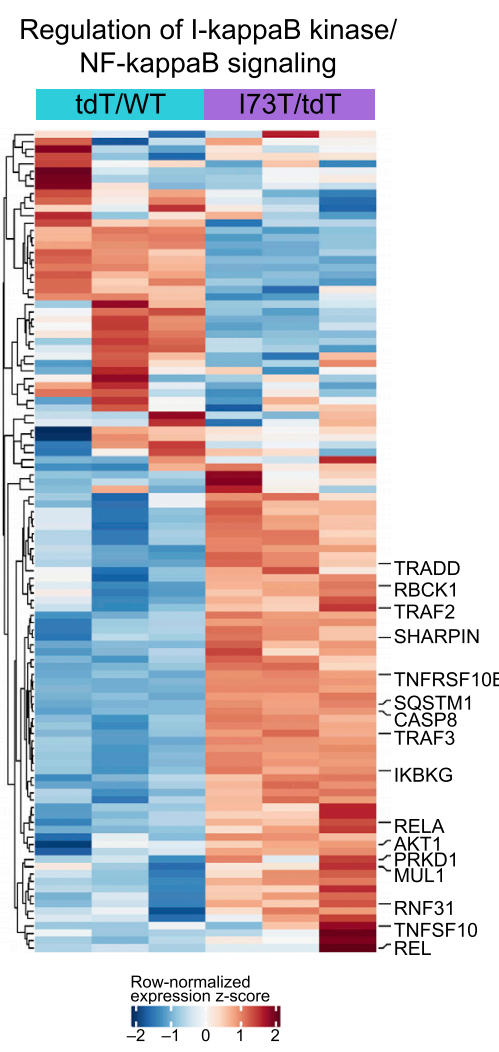

G

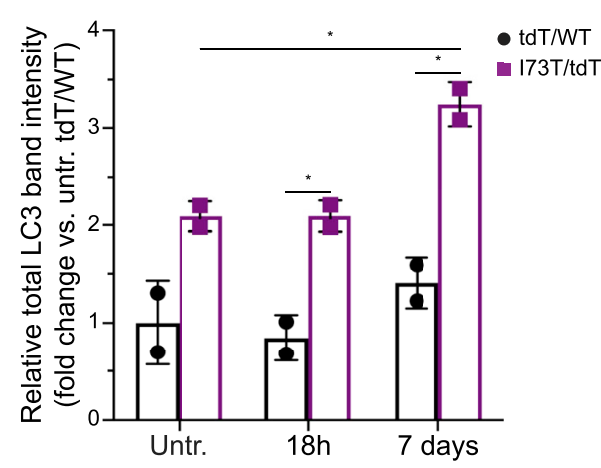

C

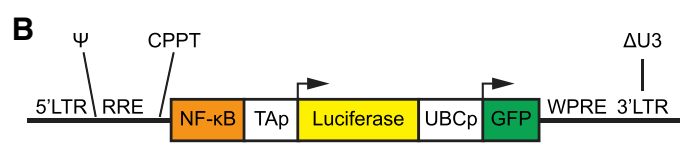
Bright field SFTPC ${ }^{\text {tdTomato }}$ UBCGFP

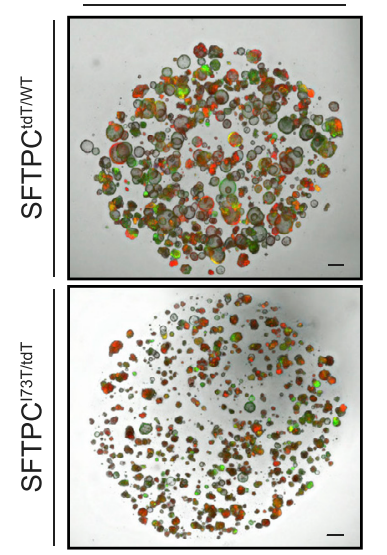

D

E
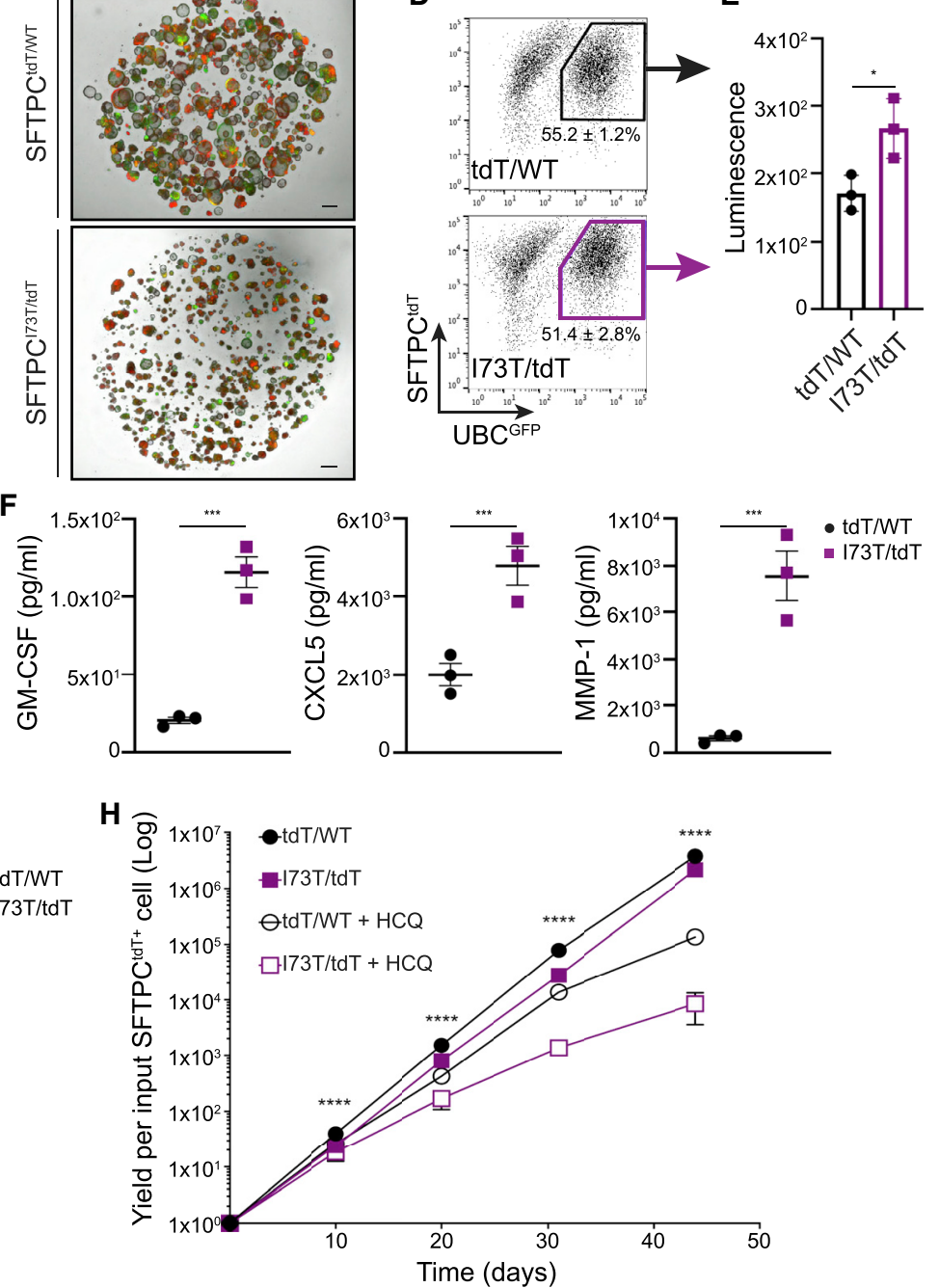

Figure 7. SFTPC ${ }^{173 T / t d T}$ iAEC2s display activation of the NF- $\kappa B$ pathway and are more susceptible to hydroxychloroquine treatment (A) Unsupervised hierarchical clustered heatmap of differentially expressed proteins (FDR $<0.05$ ) in the depicted GO biological process gene set (row normalized Z-score); a subset of differentially expressed proteins is highlighted with large font.

(B) Schematic of NF-кB-luc-GFP lentiviral construct. LTR, lentiviral long terminal repeats; RRE, rev responsive element; CPPT, central polypurine tract; WPRE, Woodchuck hepatitis virus post-transcriptional regulatory element; $\Delta \mathrm{U} 3$, deleted U3 region for in vivo inactivation of the viral LTR promoter; $\Psi$, Psi lentiviral packaging sequence; GFP, green fluorescence protein.

(C) Representative live-cell imaging of lentivirally transduced SFTPC ${ }^{\text {tdT/WT }}$ and SFTPC ${ }^{173 T / d \mathrm{dT}}$ iAEC2s (bright-field/tdTomato/GFP overlay). Scale bars, $500 \mu \mathrm{m}$.

(D) Sort gates used to purify transduced (GFP ${ }^{+}$SFTPC ${ }^{\text {tdT/WT }}$ and SFTPC ${ }^{173 T / t d T}$ iAEC2s.

(E) Bioluminescence quantification shows increased luciferase activity in SFTPC ${ }^{173 T / \text { td }}$ versus SFTPC ${ }^{\text {tdT/WT }}$ iAEC2s. ${ }^{*} p<0.05,{ }^{* *} p<0.01,{ }^{* * *} p<0.001$ by unpaired, two-tailed Student's $t$ test for all panels.

(F) Luminex analysis of supernatants collected from SFTPC ${ }^{173 T / \mathrm{tdT}}$ and SFTPC ${ }^{\mathrm{tdT} / \mathrm{NT}} \mathrm{iAEC} 2 \mathrm{~s}$ for the NF-KB target proteins GM-CSF, CXCL5, and MMP-1. ${ }^{*} p<0.05,{ }^{* \star} p<0.01,{ }^{* \star *} p<0.001$ by unpaired, two-tailed Student's $t$ test for all panels.

(G) SFTPC ${ }^{\text {tdT/WT }}$ or SFTPC ${ }^{173 T / t d T}$ iAEC2s were treated with hydroxychloroquine $(\mathrm{HCQ} ; 10 \mu \mathrm{M})$ or vehicle $\left(\mathrm{ddH}_{2} \mathrm{O}\right)$ for $18 \mathrm{~h}$ or 7 days. Lysates were subjected to western blotting for LC3 with $\beta$ actin as loading control. Densitometric quantification is shown. See also Figure $S 5 .{ }^{\star} p<0.05,{ }^{\star \star} p<0.01,{ }^{\star \star \star} p<0.001$ by unpaired, two-tailed Student's t test for all panels. 
AEC2s when compared to WT AEC2s. Together, these data suggest that SFTPC mutant AEC2s demonstrate metabolic reprogramming from oxidative phosphorylation to glycolysis, a process that appears to be time-dependent.

\section{SFTPC ${ }^{173 T}$ iAEC2s elicit an inflammatory response} The proteomic analysis revealed upregulation of the NF- $\kappa B$ pathway in SFTPC ${ }^{173 T}$ mutant iAEC2s compared to their corrected counterparts (Figure $7 \mathrm{~A}$ ). Because autophagy inhibition has been shown to lead to activation of the NF- $\kappa B$ pathway via upregulation of p62 (Hill et al., 2019), we sought to further validate the proteomic findings. We utilized a lentiviral vector (lenti-NF-кB-luc-GFP) (Figure 7B) that we previously engineered to enable independent simultaneous tracking of transduced $\left(\mathrm{GFP}^{+}\right)$cells by flow cytometry and real-time assessment of NF- $\kappa B$ activation levels quantified by a luciferase reporter whose expression is regulated by a minimal promoter element preceded by consensus binding sites for the canonical NF- $\kappa B$ p50-p65 heterodimer transcription factor complex (Wilson et al., 2013). Mutant and corrected iAEC2s were infected with the lentiviral vector (MOI 20: Figure 7C), allowed to expand in culture, and transduced $\left(\mathrm{GFP}^{+}\right)$SFTPC ${ }^{\text {tdTomato+ }}$ mutant and corrected $\mathrm{iAEC2s}$ were then sorted and analyzed for luciferase expression (Figure 7D). Mutant iAEC2s demonstrated increased bioluminescence compared to corrected iAEC2s, suggesting increased canonical NF- $\kappa$ B activity in mutant iAEC2s (Figure 7E). Furthermore, mutant iAEC2s secreted higher levels of the NF- $\kappa B$ target proteins GM-CSF, CXCL5, and MMP-1 quantified by Luminex analysis of supernatants, compared to corrected iAEC2s (Figure 7F). Taken together, these findings suggest mutant iAEC2s are an important proinflammatory hub with resultant secretion of inflammatory mediators via activation of the NF- $\kappa B$ pathway.

\section{Treatment of SFTPC ${ }^{173 T}$ iAEC2s with}

hydroxychloroquine results in aggravation of autophagy perturbations and metabolic reprogramming

To apply our iPSC-derived model system as a preclinical disease model for drug testing, in a proof-of-principle experiment we assessed the effect of hydroxychloroquine (HCQ) on SFTPC ${ }^{173 T}$ expressing iAEC2s. HCQ is a medication commonly used either alone or in combination with corticosteroids in pediatric patients with genetic disorders of surfactant dysfunction affecting AEC2s (including SFTPC ${ }^{173 T}$ ) and resulting in chILD. The use of HCQ in this patient population is based primarily on evidence from case reports or small cohort studies with variable results (Braun et al., 2015; Klay et al., 2018; Kröner et al., 2015). HCQ is a lysosomotropic medication that accumulates in lysosomes inhibiting lysosomal activity and autophagy (Mauthe et al., 2018; Schrezenmeier and Dörner, 2020) raising the possibility it may aggravate the autophagic block already present in SFTPC ${ }^{173 T}$ expressing iAEC2s. Indeed, treatment of mutant iAEC2s with
HCQ $(10 \mu \mathrm{M})$ for 7 days resulted in increased accumulation of LC3 in HCQ-treated compared to untreated iAEC2s (Figures $7 \mathrm{G}$ and S5A). Notably, treatment of corrected iAEC2s with $\mathrm{HCQ}$ under the same conditions did not result in significant changes of total LC3 suggesting that mutant iAEC2s are more sensitive to HCQ's lysosomotropic effect. Long-term treatment of mutant iAEC2s with $\mathrm{HCQ}(10 \mu \mathrm{M})$ resulted in further reduction of the already diminished self-renewal capacity of these cells (Figure $7 \mathrm{H}$ ), whereas both mutant and corrected iAEC2s retained expression of the SFTPC ${ }^{\text {tdTomato }}$ reporter (Figure S5B). Furthermore, mutant iAEC2s treated with HCQ for 7 days demonstrated a significant reduction in OCR/ECAR ratio assessed after the administration of oligomycin and FCCP, compared to untreated iAEC2s (Figure S5C). This suggests a shift toward glycolysis, revealed after pharmacological manipulation of energy supply and demand and reminiscent of the observations made earlier for aged mutant iAEC2s (Figure 6). Similar to LC3 levels, HCQ treatment had no effect on the OCR/ECAR ratio in corrected iAEC2s (Figure S5C).

\section{DISCUSSION}

Our results suggest that patient-specific iAEC2s can serve as a human preclinical disease model, revealing key components of the epithelial-intrinsic pathophysiology of human AEC2 dysfunction and providing a platform to test therapeutics. $\mathrm{Pa}-$ tient-derived iAEC2s expressing mutant SFTPC ${ }^{173 T}$ in this model exhibit misprocessed and mistrafficked pro-SFTPC protein, recapitulating the mistrafficking occurring in vivo in the donor's AEC2s. The accumulation of misprocessed proSFTPC, as a result of mutant SFTPC ${ }^{173 T}$ expressed from the endogenous human locus in AEC2-like cells, extends similar observations previously made based on forced overexpression of mutant protein in heterologous cell lines (Brasch et al., 2004; Galetskiy et al., 2008; Hawkins et al., 2015; Stewart et al., 2012), profiles of patient bronchoalveolar lavage samples (Brasch et al., 2004), and recently in an in vivo mouse model (Nureki et al., 2018).

The iAEC2 platform also offers the ability to temporally model both the early changes coinciding with accumulation of mutant SFTPC forms and the resulting downstream sequence of events leading to acquisition of a dysfunctional AEC2 phenotype. Multiomic profiling of syngeneically controlled mutant versus corrected iAEC2s identified similarities at an early time point but more distinct phenotypes emerged with culture expansion suggesting a time-dependent phenotype. This time course is in agreement with the Sftpc ${ }^{173 T}$ mouse, where an age-dependent lung-diseased phenotype emerged simultaneously with the gradual accumulation of mutant pro-SFTPC forms (Nureki et al., 2018).

Among the many cellular programs disrupted in the iAEC2 model, our findings suggest an early perturbation in autophagy.

(H) Graph showing yield in cell number per input SFTPC ${ }^{\text {tdT/WT }}$ or SFTPC ${ }^{173 T / t d T}$ iAEC2 with or without treatment with $\mathrm{HCQ}(10 \mu \mathrm{M})$. ${ }^{\star \star \star \star} \mathrm{p}<0.0001$ across all groups by one-way ANOVA; $p<0.001$ for $\mathrm{d} 10, \mathrm{p}<0.01$ for $\mathrm{d} 20, \mathrm{p}<0.0001$ for $\mathrm{d} 31$, and $\mathrm{p}<0.05$ for d44 for corrected versus mutant iAEC2s by unpaired, two-tailed Student's t test.

Mean \pm SD is shown; $n=3(E$ and $F)$ or $n=2(G)$ experimental replicates of independent wells of a differentiation or $n=3(H)$ biological replicates of independent differentiations. 
First, unbiased signaling pathway analysis identified the lysosomal/autophagy pathway as being differentially upregulated in mutant iAEC2s. We validated these observations by a variety of independent static and dynamic approaches (Figure 5) that documented induction of autophagosome formation (flux) and defined a late block in autophagy at the level of autophagosome turnover, evidenced by accumulation of LC3 and p62 and incomplete response to torin in mutant iAEC2s. Importantly, we validated these observations in vivo in primary mouse $\mathrm{Sftpc}^{173 T}$ AEC2s that are not hypo-proliferative, suggesting that the accumulation of misprocessed and mistrafficked pro-SFTPC was responsible for the observed phenotype rather than the proliferative differences between mutant and corrected iAEC2s. A dysregulated autophagic response has been shown in IPF lungs (Araya et al., 2013; Hill et al., 2019; Patel et al., 2012) and has been postulated to lead to epithelial cell senescence and myofibroblast differentiation (Araya et al., 2013; Hill et al., 2019). Although it remains unclear mechanistically how SFTPC buildup/misprocessing leads to autophagy perturbations, we speculate that it is likely related to failure of lysosomal fusion because lysosomal acidification appears intact in mutant iAEC2s. The resultant proteostasis perturbations and alterations in cellular quality control resulted in diminished progenitor potential as evidenced by reduced self-renewal capacity. Furthermore, the paucity of AEC1s in the donor's lung explant (Figure S2A), similar to findings described in our Sftpc ${ }^{173 T}$ knockin mice (Nureki et al., 2018), point to disruption of normal $A E C 2 / A E C 1$ homeostasis as a result of AEC2 dysfunction. Ineffectual AEC2-to-AEC1 differentiation and emergence of a transitional cell state have been described in IPF (Jiang et al., 2020; Riemondy et al., 2019). In addition, selective AEC2 ablation (Barkauskas et al., 2013; Garcia et al., 2016; Sisson et al., 2010) or AEC2 senescence induction via conditional deletion of $\operatorname{Sin} 3 a$ (Yao et al., 2020) in mice have resulted in progressive pulmonary fibrosis, either spontaneously or after bleomycin exposure. Therefore, our results agree with the growing body of literature suggesting that dysfunctional AEC2s could initiate the fibrotic cascade.

Because autophagy plays an important role in selective degradation of dysfunctional mitochondria, this may explain our observation that mutant iAEC2s displaying an autophagy phenotype accumulated dysmorphic and dysfunctional mitochondria in a time-dependent manner, changes previously described in primary AEC2s from patients with IPF and aged mice (Bueno et al., 2015). We further validated the mitochondrial dysfunction and metabolic reprogramming observed in mutant iAEC2s in primary mouse Sftpc ${ }^{173 T}$ AEC2s. Assessing the bioenergetics of human AEC2s has not been previously possible given the challenges in culturing primary AEC2s.

In addition to detailed alterations in cell quality control and metabolism, our data also provide a plausible explanation of how a dysfunctional AEC2 signals to surrounding cells to initiate inflammatory and profibrotic cascades, because the NF- $\kappa \mathrm{B}$ pathway was found to be differentially upregulated in mutant compared to corrected iAEC2s. Furthermore, higher levels of the NF- $\kappa B$ target proteins GM-CSF, CXCL5, and MMP-1 were identified in the supernatants of mutant compared to corrected iAEC2s. Importantly, GM-CSF is a strong chemoattractant for profibrotic Ly6c ${ }^{\text {hi }}$ monocytes that have been implicated in the pathogenesis of lung fibrosis (Misharin et al., 2017; Venosa et al., 2019).

Finally, in a proof-of-principle experiment, we tested the application of our model system as a preclinical human disease drug testing platform by assessing the effect of HCQ on SFTPC ${ }^{173 T_{-}}$ expressing cells. Treatment of mutant iAEC2s resulted in aggravation of the observed autophagy perturbations, further reduction of the already diminished self-renewal capacity, and metabolic reprogramming toward glycolysis. Taken together, the findings from our epithelial-only model system suggest that treatment with $\mathrm{HCQ}$ could be detrimental to patients carrying the SFTPC ${ }^{173 T}$ variant, although a pleiotropic effect resulting from HCQ's effect on immune cells or beneficial effects on other cell types in vivo cannot be excluded.

Prior reports (Jacob et al., 2017; Korogi et al., 2019; Strikoudis et al., 2019) have provided proof-of-concept that ESC- or iPSC-derived lung epithelia carrying certain mutations could model some aspects of either adult or pediatric ILD, such as inflammatory activation. We believe the current study adds to these prior reports by addressing some of the many mechanisms associated with a cell autonomous, epithelialintrinsic toxic gain-of-function in purified patient-specific iAEC2s, including detailed profiles of the perturbations in protein trafficking, autophagy, mitochondrial morphology, and function.

In summary, our epithelial-only patient-specific iAEC2 model system not only recapitulates key observations made previously in heterologous cell lines and in vivo mouse models (as well as in vivo in the patient from whom the iPSCs were generated) but also provides new insights into the potential role of AEC2s in the inception of pulmonary fibrosis and serves as a preclinical platform to test therapeutics.

\section{STAR $\star$ METHODS}

Detailed methods are provided in the online version of this paper and include the following:

- KEY RESOURCES TABLE

- RESOURCE AVAILABILITY

- Lead contact

- Materials availability

- Data and code availability

- EXPERIMENTAL MODEL AND SUBJECT DETAILS

O iPSC line generation and maintenance

$\bigcirc$ Generation of gene-edited syngeneic iPSC lines

- METHOD DETAILS

O iPSC directed differentiation into alveolar epithelial type 2 cells (iAEC2s)

Murine model of Sftpc ${ }^{173 T}$

O Mouse AEC2 isolation

Flow cytometry and Fluorescence Activated Cell Sorting (FACS)

Immunofluorescence microscopy

- Cell proliferation analysis

- Transmission electron microscopy

Other staining 
$\bigcirc$ Quantification of autophagic flux

Western Blot Analysis

O Respirometry assays

O Mitochondrial content determination

$\bigcirc$ Quantification of NF- $\kappa$ B signaling

O Luminex analysis

O Reverse Transcription Quantitative Polymerase Chain Reaction (RT-qPCR)

Isolation of Human Fetal Lung Epithelium

O RNA sequencing and bioinformatic analyses

O Proteomic and phosphoproteomic analysis

- QUANTIFICATION AND STATISTICAL ANALYSIS

\section{SUPPLEMENTAL INFORMATION}

Supplemental information can be found online at https://doi.org/10.1016/j. celrep.2021.109636.

\section{ACKNOWLEDGMENTS}

The authors wish to thank all members of the Kotton, Beers, Emili, and Shirihai Labs for insightful discussions. We thank Anne Hinds of the Boston University Pulmonary Center for histology technical support. We thank Yuriy Alekseyev of the Boston University School of Medicine (BUSM) Single Cell Sequencing Core, supported by NIH (1UL1TR001430), and Brian R. Tilton of the BUSM Flow Cytometry Core. We are grateful to Greg Miller and Marianne James of the Boston University Center for Regenerative Medicine (CReM) for maintenance and characterization of patient-specific iPSCs, supported by NIH (NO1 75N92020C00005 and U01TR001810). We thank Michael Morley and Edward E. Morrisey of the University of Pennsylvania for access to their bioinformatics portal for analyses of bulk RNA-seq datasets. The graphical abstract, the autophagy pathway schematic, and the mouse and human cartoons were created with BioRender.com. This work was supported by the I.M. Rosenzweig Junior Investigator Award from The Pulmonary Fibrosis Foundation (to K.-D.A.), an IDEAL Consortium grant from Celgene/ Bristol Myers Squibb (D.N.K.), the Albert M. Rose Established Investigator Award from the Pulmonary Fibrosis Foundation (to M.F.B.), the Department of Veterans Affairs Merit Review (1101BX001176 to M.F.B.), and $\mathrm{NIH}$ (U01HL148692, U01HL134745, U01HL134766, U01HL152976, and R01HL095993 to D.N.K., U01HL152970 and RO1HL119436 to M.F.B., and K24HL143281 to L.R.Y.). A.E. acknowledges generous startup funding to the operations of the CNSB from Boston University.

\section{AUTHOR CONTRIBUTIONS}

K.-D.A., M.F.B., and D.N.K. conceived the work. K.-D.A., A.P., E.P.T., X.V., M.L., A.E., S.H.G., O.S.S., M.F.B., and D.N.K. designed experiments. K.-D.A., S.J.R., A.P., E.P.T., R.A.-P., S.M., L.R.R., R.M.H., O.T.H., K.M., M.V., S.K., and A.M.T.-L. conducted experiments and analyzed data. J.C.J. and D.N.K. designed the targeting strategy and generated the iPSC lines. C.V.-M. and B.C.B. performed bioinformatics analysis. J.A.W., F.S.C., A.H., and L.R.Y. provided patient samples. S.H.G. provided human fetal lung samples. K.-D.A., M.F.B., and D.N.K. prepared the original draft. K.-D.A., D.N.K., M.F.B., S.H.G., A.P., E.P.T., F.S.C., J.A.W., B.C.B., and R.M.H. edited the manuscript. All authors reviewed and approved the final version prior to submission.

\section{DECLARATION OF INTERESTS}

The authors declare no competing interests.

Received: November 18, 2020

Revised: April 28, 2021

Accepted: August 6, 2021

Published: August 31, 2021

\section{REFERENCES}

Acin-Perez, R., Benador, I.Y., Petcherski, A., Veliova, M., Benavides, G.A., Lagarrigue, S., Caudal, A., Vergnes, L., Murphy, A.N., Karamanlidis, G., et al. (2020). A novel approach to measure mitochondrial respiration in frozen biological samples. EMBO J. 39, e104073.

Araya, J., Kojima, J., Takasaka, N., Ito, S., Fujii, S., Hara, H., Yanagisawa, H., Kobayashi, K., Tsurushige, C., Kawaishi, M., et al. (2013). Insufficient autophagy in idiopathic pulmonary fibrosis. Am. J. Physiol. Lung Cell. Mol. Physiol. 304, L56-L69.

Assali, E.A., Shlomo, D., Zeng, J., Taddeo, E.P., Trudeau, K.M., Erion, K.A., Colby, A.H., Grinstaff, M.W., Liesa, M., Las, G., and Shirihai, O.S. (2019). Nanoparticle-mediated lysosomal reacidification restores mitochondrial turnover and function in $\beta$ cells under lipotoxicity. FASEB J. 33, 4154-4165.

Atochina-Vasserman, E.N., Bates, S.R., Zhang, P., Abramova, H., Zhang, Z., Gonzales, L., Tao, J.-Q., Gochuico, B.R., Gahl, W., Guo, C.-J., et al. (2011). Early alveolar epithelial dysfunction promotes lung inflammation in a mouse model of Hermansky-Pudlak syndrome. Am. J. Respir. Crit. Care Med. 184, 449-458.

Barkauskas, C.E., and Noble, P.W. (2014). Cellular mechanisms of tissue fibrosis. 7. New insights into the cellular mechanisms of pulmonary fibrosis. Am. J. Physiol. Cell Physiol. 306, C987-C996.

Barkauskas, C.E., Cronce, M.J., Rackley, C.R., Bowie, E.J., Keene, D.R., Stripp, B.R., Randell, S.H., Noble, P.W., and Hogan, B.L.M. (2013). Type 2 alveolar cells are stem cells in adult lung. J. Clin. Invest. 123, 3025-3036.

Beers, M.F., and Lomax, C. (1995). Synthesis and processing of hydrophobic surfactant protein C by isolated rat type II cells. Am. J. Physiol. 269, L744L753.

Beers, M.F., and Mulugeta, S. (2005). Surfactant protein C biosynthesis and its emerging role in conformational lung disease. Annu. Rev. Physiol. 67, 663-696.

Beers, M.F., Bates, S.R., and Fisher, A.B. (1992). Differential extraction for the rapid purification of bovine surfactant protein B. Am. J. Physiol. 262, L773L778.

Beers, M.F., Kim, C.Y., Dodia, C., and Fisher, A.B. (1994). Localization, synthesis, and processing of surfactant protein SP-C in rat lung analyzed by epitopespecific antipeptide antibodies. J. Biol. Chem. 269, 20318-20328.

Beers, M.F., Hawkins, A., Maguire, J.A., Kotorashvili, A., Zhao, M., Newitt, J.L., Ding, W., Russo, S., Guttentag, S., Gonzales, L., and Mulugeta, S. (2011). A nonaggregating surfactant protein $\mathrm{C}$ mutant is misdirected to early endosomes and disrupts phospholipid recycling. Traffic 12, 1196-1210.

Bock, C., Kiskinis, E., Verstappen, G., Gu, H., Boulting, G., Smith, Z.D., Ziller, M., Croft, G.F., Amoroso, M.W., Oakley, D.H., et al. (2011). Reference Maps of human ES and iPS cell variation enable high-throughput characterization of pluripotent cell lines. Cell 144, 439-452.

Borok, Z., Danto, S.I., Lubman, R.L., Cao, Y., Williams, M.C., and Crandall, E.D. (1998). Modulation of t1alpha expression with alveolar epithelial cell phenotype in vitro. Am. J. Physiol. 275, L155-L164.

Boulting, G.L., Kiskinis, E., Croft, G.F., Amoroso, M.W., Oakley, D.H., Wainger, B.J., Williams, D.J., Kahler, D.J., Yamaki, M., Davidow, L., et al. (2011). A functionally characterized test set of human induced pluripotent stem cells. Nat. Biotechnol. 29, 279-286.

Brasch, F., Ten Brinke, A., Johnen, G., Ochs, M., Kapp, N., Müller, K.M., Beers, M.F., Fehrenbach, H., Richter, J., Batenburg, J.J., and Bühling, F. (2002) Involvement of cathepsin $\mathrm{H}$ in the processing of the hydrophobic surfactantassociated protein C in type II pneumocytes. Am. J. Respir. Cell Mol. Biol. $26,659-670$.

Brasch, F., Griese, M., Tredano, M., Johnen, G., Ochs, M., Rieger, C., Mulugeta, S., Müller, K.M., Bahuau, M., and Beers, M.F. (2004). Interstitial lung disease in a baby with a de novo mutation in the SFTPC gene. Eur. Respir. J. 24, 30-39. 
Braun, S., Ferner, M., Kronfeld, K., and Griese, M. (2015). Hydroxychloroquine in children with interstitial (diffuse parenchymal) lung diseases. Pediatr. Pulmonol. 50, 410-419.

Bueno, M., Lai, Y.-C., Romero, Y., Brands, J., St Croix, C.M., Kamga, C., Corey, C., Herazo-Maya, J.D., Sembrat, J., Lee, J.S., et al. (2015). PINK1 deficiency impairs mitochondrial homeostasis and promotes lung fibrosis. J. Clin. Invest. 125, 521-538.

Cottin, V., Reix, P., Khouatra, C., Thivolet-Béjui, F., Feldmann, D., and Cordier, J.-F. (2011). Combined pulmonary fibrosis and emphysema syndrome associated with familial SFTPC mutation. Thorax 66, 918-919.

Crossno, P.F., Polosukhin, V.V., Blackwell, T.S., Johnson, J.E., Markin, C., Moore, P.E., Worrell, J.A., Stahlman, M.T., Phillips, J.A., 3rd, Loyd, J.E., et al. (2010). Identification of early interstitial lung disease in an individual with genetic variations in ABCA3 and SFTPC. Chest 137, 969-973.

Deutsch, E.W., Bandeira, N., Sharma, V., Perez-Riverol, Y., Carver, J.J., Kundu, D.J., García-Seisdedos, D., Jarnuczak, A.F., Hewapathirana, S., Pullman, B.S., et al. (2020). The ProteomeXchange consortium in 2020: enabling 'big data' approaches in proteomics. Nucleic Acids Res. 48 (D1), D1145D1152.

Dobin, A., Davis, C.A., Schlesinger, F., Drenkow, J., Zaleski, C., Jha, S., Batut, P., Chaisson, M., and Gingeras, T.R. (2013). STAR: ultrafast universal RNA-seq aligner. Bioinformatics 29, 15-21.

Foster, C.D., Varghese, L.S., Skalina, R.B., Gonzales, L.W., and Guttentag, S.H. (2007). In vitro transdifferentiation of human fetal type II cells toward a type I-like cell. Pediatr. Res. 61, 404-409.

Galetskiy, D., Woischnik, M., Ripper, J., Griese, M., and Przybylski, M. (2008). Aberrant processing forms of lung surfactant proteins SP-B and SP-C revealed by high-resolution mass spectrometry. Eur. J. Mass Spectrom (Chichester) 14, 379-390.

Garcia, C.K. (2018). Insights from human genetic studies of lung and organ fibrosis. J. Clin. Invest. 128, 36-44.

Garcia, O., Hiatt, M.J., Lundin, A., Lee, J., Reddy, R., Navarro, S., Kikuchi, A., and Driscoll, B. (2016). Targeted Type 2 Alveolar Cell Depletion. A Dynamic Functional Model for Lung Injury Repair. Am. J. Respir. Cell Mol. Biol. 54, 319-330.

Gu, Z., Eils, R., and Schlesner, M. (2016). Complex heatmaps reveal patterns and correlations in multidimensional genomic data. Bioinformatics 32, $2847-$ 2849.

Habermann, A.C., Gutierrez, A.J., Bui, L.T., Yahn, S.L., Winters, N.I., Calvi, C.L., Peter, L., Chung, M.-I., Taylor, C.J., Jetter, C., et al. (2020). Single-cell RNA sequencing reveals profibrotic roles of distinct epithelial and mesenchymal lineages in pulmonary fibrosis. Sci. Adv. 6, eaba1972.

Hawkins, A., Guttentag, S.H., Deterding, R., Funkhouser, W.K., Goralski, J.L., Chatterjee, S., Mulugeta, S., and Beers, M.F. (2015). A non-BRICHOS SFTPC mutant (SP-Cl73T) linked to interstitial lung disease promotes a late block in macroautophagy disrupting cellular proteostasis and mitophagy. Am. J. Physiol. Lung Cell. Mol. Physiol. 308, L33-L47.

Hawkins, F., Kramer, P., Jacob, A., Driver, I., Thomas, D.C., McCauley, K.B., Skvir, N., Crane, A.M., Kurmann, A.A., Hollenberg, A.N., et al. (2017). Prospective isolation of NKX2-1-expressing human lung progenitors derived from pluripotent stem cells. J. Clin. Invest. 127, 2277-2294.

Hill, C., Li, J., Liu, D., Conforti, F., Brereton, C.J., Yao, L., Zhou, Y., Alzetani, A., Chee, S.J., Marshall, B.G., et al. (2019). Autophagy inhibition-mediated epithelial-mesenchymal transition augments local myofibroblast differentiation in pulmonary fibrosis. Cell Death Dis. 10, 591.

Hurley, K., Ding, J., Villacorta-Martin, C., Herriges, M.J., Jacob, A., Vedaie, M., Alysandratos, K.-D., Sun, Y.L., Lin, C., Werder, R.B., et al. (2020). Reconstructed Single-Cell Fate Trajectories Define Lineage Plasticity Windows during Differentiation of Human PSC-Derived Distal Lung Progenitors. Cell Stem Cell 26, 593-608.e8.

Jacob, A., Morley, M., Hawkins, F., McCauley, K.B., Jean, J.C., Heins, H., Na, C.-L., Weaver, T.E., Vedaie, M., Hurley, K., et al. (2017). Differentiation of Hu- man Pluripotent Stem Cells into Functional Lung Alveolar Epithelial Cells. Cell Stem Cell 21, 472-488.e10.

Jacob, A., Vedaie, M., Roberts, D.A., Thomas, D.C., Villacorta-Martin, C., Alysandratos, K.-D., Hawkins, F., and Kotton, D.N. (2019). Derivation of self-renewing lung alveolar epithelial type II cells from human pluripotent stem cells. Nat. Protoc. 14, 3303-3332.

Jiang, P., Gil de Rubio, R., Hrycaj, S.M., Gurczynski, S.J., Riemondy, K.A., Moore, B.B., Omary, M.B., Ridge, K.M., and Zemans, R.L. (2020). Ineffectual Type 2-to-Type 1 Alveolar Epithelial Cell Differentiation in Idiopathic Pulmonary Fibrosis: Persistence of the KRT8 ${ }^{\text {hi }}$ Transitional State. Am. J. Respir. Crit. Care Med. 201, 1443-1447.

Katzen, J., Wagner, B.D., Venosa, A., Kopp, M., Tomer, Y., Russo, S.J., Headen, A.C., Basil, M.C., Stark, J.M., Mulugeta, S., et al. (2019). An SFTPC BRICHOS mutant links epithelial ER stress and spontaneous lung fibrosis. JCI Insight 4, e126125.

Katzenstein, A.L. (1985). Pathogenesis of "fibrosis" in interstitial pneumonia: an electron microscopic study. Hum. Pathol. 16, 1015-1024.

Kim, K., Doi, A., Wen, B., Ng, K., Zhao, R., Cahan, P., Kim, J., Aryee, M.J., Ji, H., Ehrlich, L.I.R., et al. (2010). Epigenetic memory in induced pluripotent stem cells. Nature 467, 285-290.

Klay, D., Hoffman, T.W., Harmsze, A.M., Grutters, J.C., and van Moorsel, C.H.M. (2018). Systematic review of drug effects in humans and models with surfactant-processing disease. Eur. Respir. Rev. 27, 170135.

Klionsky, D.J., Abdelmohsen, K., Abe, A., Abedin, M.J., Abeliovich, H., Acevedo Arozena, A., Adachi, H., Adams, C.M., Adams, P.D., Adeli, K., et al. (2016). Guidelines for the use and interpretation of assays for monitoring autophagy (3rd edition). Autophagy 12, 1-222.

Korogi, Y., Gotoh, S., Ikeo, S., Yamamoto, Y., Sone, N., Tamai, K., Konishi, S., Nagasaki, T., Matsumoto, H., Ito, I., et al. (2019). In Vitro Disease Modeling of Hermansky-Pudlak Syndrome Type 2 Using Human Induced Pluripotent Stem Cell-Derived Alveolar Organoids. Stem Cell Reports 12 431-440.

Korotkevich, G., Sukhov, V., and Sergushichev, A. (2019). Fast gene set enrichment analysis. bioRxiv. https://doi.org/10.1101/060012.

Kröner, C., Reu, S., Teusch, V., Schams, A., Grimmelt, A.-C., Barker, M., Brand, J., Gappa, M., Kitz, R., Kramer, B.W., et al. (2015). Genotype alone does not predict the clinical course of SFTPC deficiency in paediatric patients. Eur. Respir. J. 46, 197-206.

Kropski, J.A., Blackwell, T.S., and Loyd, J.E. (2015). The genetic basis of idiopathic pulmonary fibrosis. Eur. Respir. J. 45, 1717-1727.

Law, C.W., Alhamdoosh, M., Su, S., Dong, X., Tian, L., Smyth, G.K., and Ritchie, M.E. (2016). RNA-seq analysis is easy as 1-2-3 with limma, Glimma and edgeR. F1000Res. 5, 1408.

Lederer, D.J., and Martinez, F.J. (2018). Idiopathic Pulmonary Fibrosis. N. Engl. J. Med. 378, 1811-1823.

Maguire, J.A., Mulugeta, S., and Beers, M.F. (2012). Multiple ways to die: delineation of the unfolded protein response and apoptosis induced by Surfactant Protein C BRICHOS mutants. Int. J. Biochem. Cell Biol. 44, 101-112.

Massaro, G.D., Gail, D.B., and Massaro, D. (1975). Lung oxygen consumption and mitochondria of alveolar epithelial and endothelial cells. J. Appl. Physiol. 38, 588-592.

Mauthe, M., Orhon, I., Rocchi, C., Zhou, X., Luhr, M., Hijlkema, K.-J., Coppes, R.P., Engedal, N., Mari, M., and Reggiori, F. (2018). Chloroquine inhibits autophagic flux by decreasing autophagosome-lysosome fusion. Autophagy 14 , 1435-1455.

Misharin, A.V., Morales-Nebreda, L., Reyfman, P.A., Cuda, C.M., Walter, J.M., McQuattie-Pimentel, A.C., Chen, C.-I., Anekalla, K.R., Joshi, N., Williams, K.J.N., et al. (2017). Monocyte-derived alveolar macrophages drive lung fibrosis and persist in the lung over the life span. J. Exp. Med. 214, 2387-2404.

Mulugeta, S., Nureki, S., and Beers, M.F. (2015). Lost after translation: insights from pulmonary surfactant for understanding the role of alveolar epithelial 
dysfunction and cellular quality control in fibrotic lung disease. Am. J. Physiol. Lung Cell. Mol. Physiol. 309, L507-L525.

Ni, H.-M., Bockus, A., Wozniak, A.L., Jones, K., Weinman, S., Yin, X.-M., and Ding, W.-X. (2011). Dissecting the dynamic turnover of GFP-LC3 in the autolysosome. Autophagy 7, 188-204.

Nogee, L.M., Dunbar, A.E., 3rd, Wert, S.E., Askin, F., Hamvas, A., and Whitsett, J.A. (2001). A mutation in the surfactant protein $C$ gene associated with familial interstitial lung disease. N. Engl. J. Med. 344, 573-579.

Nureki, S.-I., Tomer, Y., Venosa, A., Katzen, J., Russo, S.J., Jamil, S., Barrett, M., Nguyen, V., Kopp, M., Mulugeta, S., and Beers, M.F. (2018). Expression of mutant Sftpc in murine alveolar epithelia drives spontaneous lung fibrosis. J. Clin. Invest. 128, 4008-4024.

Ono, S., Tanaka, T., Ishida, M., Kinoshita, A., Fukuoka, J., Takaki, M., Sakamoto, N., Ishimatsu, Y., Kohno, S., Hayashi, T., et al. (2011). Surfactant protein C G100S mutation causes familial pulmonary fibrosis in Japanese kindred. Eur. Respir. J. 38, 861-869.

Patel, A.S., Lin, L., Geyer, A., Haspel, J.A., An, C.H., Cao, J., Rosas, I.O., and Morse, D. (2012). Autophagy in idiopathic pulmonary fibrosis. PLoS ONE 7, e41394.

Perez-Riverol, Y., Csordas, A., Bai, J., Bernal-Llinares, M., Hewapathirana, S., Kundu, D.J., Inuganti, A., Griss, J., Mayer, G., Eisenacher, M., et al. (2019). The PRIDE database and related tools and resources in 2019: improving support for quantification data. Nucleic Acids Res. 47 (D1), D442-D450.

Raghu, G., Chen, S.-Y., Yeh, W.-S., Maroni, B., Li, Q., Lee, Y.-C., and Collard, H.R. (2014). Idiopathic pulmonary fibrosis in US Medicare beneficiaries aged 65 years and older: incidence, prevalence, and survival, 2001-11. Lancet Respir. Med. 2, 566-572.

Raghu, G., Chen, S.-Y., Hou, Q., Yeh, W.-S., and Collard, H.R. (2016). Incidence and prevalence of idiopathic pulmonary fibrosis in US adults 18-64 years old. Eur. Respir. J. 48, 179-186.

Reimand, J., Isserlin, R., Voisin, V., Kucera, M., Tannus-Lopes, C., Rostamianfar, A., Wadi, L., Meyer, M., Wong, J., Xu, C., et al. (2019). Pathway enrichment analysis and visualization of omics data using g:Profiler, GSEA, Cytoscape and EnrichmentMap. Nat. Protoc. 14, 482-517.

Riemondy, K.A., Jansing, N.L., Jiang, P., Redente, E.F., Gillen, A.E., Fu, R., Miller, A.J., Spence, J.R., Gerber, A.N., Hesselberth, J.R., and Zemans, R.L. (2019). Single cell RNA sequencing identifies TGF $\beta$ as a key regenerative cue following LPS-induced lung injury. JCI Insight 5, e123637.

Ritchie, M.E., Phipson, B., Wu, D., Hu, Y., Law, C.W., Shi, W., and Smyth, G.K. (2015). limma powers differential expression analyses for RNA-sequencing and microarray studies. Nucleic Acids Res. 43, e47.

Schrezenmeier, E., and Dörner, T. (2020). Mechanisms of action of hydroxychloroquine and chloroquine: implications for rheumatology. Nat. Rev. Rheumatol. 16, 155-166.

Selman, M., and Pardo, A. (2014). Revealing the pathogenic and aging-related mechanisms of the enigmatic idiopathic pulmonary fibrosis. an integral model. Am. J. Respir. Crit. Care Med. 189, 1161-1172.

Serra, M., Alysandratos, K.-D., Hawkins, F., McCauley, K.B., Jacob, A., Choi, J., Caballero, I.S., Vedaie, M., Kurmann, A.A., Ikonomou, L., et al. (2017). Pluripotent stem cell differentiation reveals distinct developmental pathways regulating lung- versus thyroid-lineage specification. Development 144, 3879-3893.

Sisson, T.H., Mendez, M., Choi, K., Subbotina, N., Courey, A., Cunningham, A., Dave, A., Engelhardt, J.F., Liu, X., White, E.S., et al. (2010). Targeted injury of type II alveolar epithelial cells induces pulmonary fibrosis. Am. J. Respir. Crit. Care Med. 181, 254-263.

Somers, A., Jean, J.-C., Sommer, C.A., Omari, A., Ford, C.C., Mills, J.A., Ying, L., Sommer, A.G., Jean, J.M., Smith, B.W., et al. (2010). Generation of transgene-free lung disease-specific human induced pluripotent stem cells using a single excisable lentiviral stem cell cassette. Stem Cells 28, 1728-1740.

Stewart, G.A., Ridsdale, R., Martin, E.P., Na, C.-L., Xu, Y., Mandapaka, K., and Weaver, T.E. (2012). 4-Phenylbutyric acid treatment rescues trafficking and processing of a mutant surfactant protein-C. Am. J. Respir. Cell Mol. Biol. 47, 324-331.

Strikoudis, A., Cieślak, A., Loffredo, L., Chen, Y.-W., Patel, N., Saqi, A., Lederer, D.J., and Snoeck, H.-W. (2019). Modeling of Fibrotic Lung Disease Using 3D Organoids Derived from Human Pluripotent Stem Cells. Cell Rep. 27, 3709-3723.e5.

Subramanian, A., Tamayo, P., Mootha, V.K., Mukherjee, S., Ebert, B.L., Gillette, M.A., Paulovich, A., Pomeroy, S.L., Golub, T.R., Lander, E.S., and Mesirov, J.P. (2005). Gene set enrichment analysis: a knowledge-based approach for interpreting genome-wide expression profiles. Proc. Natl. Acad. Sci. USA 102, 15545-15550.

Szymaniak, A.D., Mahoney, J.E., Cardoso, W.V., and Varelas, X. (2015). Crumbs3-Mediated Polarity Directs Airway Epithelial Cell Fate through the Hippo Pathway Effector Yap. Dev. Cell 34, 283-296.

Taddeo, E.P., Alsabeeh, N., Baghdasarian, S., Wikstrom, J.D., Ritou, E., Sereda, S., Erion, K., Li, J., Stiles, L., Abdulla, M., et al. (2020). Mitochondrial Proton Leak Regulated by Cyclophilin D Elevates Insulin Secretion in Islets at Nonstimulatory Glucose Levels. Diabetes 69, 131-145.

Thomas, A.Q., Lane, K., Phillips, J., 3rd, Prince, M., Markin, C., Speer, M., Schwartz, D.A., Gaddipati, R., Marney, A., Johnson, J., et al. (2002). Heterozygosity for a surfactant protein $\mathrm{C}$ gene mutation associated with usual interstitial pneumonitis and cellular nonspecific interstitial pneumonitis in one kindred. Am. J. Respir. Crit. Care Med. 165, 1322-1328.

Travis, W.D., Costabel, U., Hansell, D.M., King, T.E., Jr., Lynch, D.A., Nicholson, A.G., Ryerson, C.J., Ryu, J.H., Selman, M., Wells, A.U., et al.; ATS/ERS Committee on Idiopathic Interstitial Pneumonias (2013). An official American Thoracic Society/European Respiratory Society statement: Update of the international multidisciplinary classification of the idiopathic interstitial pneumonias. Am. J. Respir. Crit. Care Med. 188, 733-748.

Twig, G., Elorza, A., Molina, A.J.A., Mohamed, H., Wikstrom, J.D., Walzer, G. Stiles, L., Haigh, S.E., Katz, S., Las, G., et al. (2008). Fission and selective fusion govern mitochondrial segregation and elimination by autophagy. EMBO J. 27, 433-446.

van Moorsel, C.H.M., van Oosterhout, M.F.M., Barlo, N.P., de Jong, P.A., van der Vis, J.J., Ruven, H.J.T., van Es, H.W., van den Bosch, J.M.M., and Grutters, J.C. (2010). Surfactant protein C mutations are the basis of a significant portion of adult familial pulmonary fibrosis in a dutch cohort. Am. J. Respir. Crit. Care Med. 182, 1419-1425.

Venosa, A., Katzen, J., Tomer, Y., Kopp, M., Jamil, S., Russo, S.J., Mulugeta, S., and Beers, M.F. (2019). Epithelial Expression of an Interstitial Lung DiseaseAssociated Mutation in Surfactant Protein-C Modulates Recruitment and Activation of Key Myeloid Cell Populations in Mice. J. Immunol. 202, 2760-2771. Wade, K.C., Guttentag, S.H., Gonzales, L.W., Maschhoff, K.L., Gonzales, J., Kolla, V., Singhal, S., and Ballard, P.L. (2006). Gene induction during differentiation of human pulmonary type II cells in vitro. Am. J. Respir. Cell Mol. Biol. $34,727-737$.

Wilson, A.A., Kwok, L.W., Porter, E.L., Payne, J.G., McElroy, G.S., Ohle, S.J. Greenhill, S.R., Blahna, M.T., Yamamoto, K., Jean, J.C., et al. (2013). Lentiviral delivery of RNAi for in vivo lineage-specific modulation of gene expression in mouse lung macrophages. Mol. Ther. 21, 825-833.

Winters, N.I., Burman, A., Kropski, J.A., and Blackwell, T.S. (2019). Epithelial Injury and Dysfunction in the Pathogenesis of Idiopathic PulmonaryFibrosis. Am. J. Med. Sci. 357, 374-378.

Wolfe, D.M., Lee, J.-H., Kumar, A., Lee, S., Orenstein, S.J., and Nixon, R.A (2013). Autophagy failure in Alzheimer's disease and the role of defective lysosomal acidification. Eur. J. Neurosci. 37, 1949-1961.

Wu, D., and Smyth, G.K. (2012). Camera: a competitive gene set test accounting for inter-gene correlation. Nucleic Acids Res. 40, e133.

Yamamoto, A., Tagawa, Y., Yoshimori, T., Moriyama, Y., Masaki, R., and Tashiro, Y. (1998). Bafilomycin A1 prevents maturation of autophagic vacuoles by inhibiting fusion between autophagosomes and lysosomes in rat hepatoma cell line, H-4-II-E cells. Cell Struct. Funct. 23, 33-42. 
Yao, C., Guan, X., Carraro, G., Parimon, T., Liu, X., Huang, G., Mulay, A., Soukiasian, H.J., David, G., Weigt, S.S., et al. (2020). Senescence of Alveolar Type 2 Cells Drives Progressive Pulmonary Fibrosis. Am. J. Respir. Crit. Care Med. 203, 707-717.

Yin, B.W.T., Kiyamova, R., Chua, R., Caballero, O.L., Gout, I., Gryshkova, V., Bhaskaran, N., Souchelnytskyi, S., Hellman, U., Filonenko, V., et al. (2008).
Monoclonal antibody MX35 detects the membrane transporter NaPi2b (SLC34A2) in human carcinomas. Cancer Immun. 8, 3.

Yu, G., Tzouvelekis, A., Wang, R., Herazo-Maya, J.D., Ibarra, G.H., Srivastava, A., de Castro, J.P.W., Deluliis, G., Ahangari, F., Woolard, T., et al. (2018). Thyroid hormone inhibits lung fibrosis in mice by improving epithelial mitochondrial function. Nat. Med. 24, 39-49. 


\section{STAR $\star M E T H O D S$}

\section{KEY RESOURCES TABLE}

\begin{tabular}{|c|c|c|}
\hline REAGENT or RESOURCE & SOURCE & IDENTIFIER \\
\hline \multicolumn{3}{|l|}{ Antibodies } \\
\hline Rabbit polyclonal to activated caspase 3 & Sigma Aldrich & Cat\# C8487; RRID:AB_476884 \\
\hline Mouse monoclonal to $\beta$ actin & Sigma Aldrich & Cat\# A1978; RRID:AB_476692 \\
\hline Mouse monoclonal to CD26, PE conjugated (clone BA5b) & BioLegend & Cat\# 302705; RRID:AB_314289 \\
\hline $\begin{array}{l}\text { Mouse monoclonal to CD } 47 \text {, PerCP-Cy5.5 conjugated } \\
\text { (clone CC2C6) }\end{array}$ & BioLegend & Cat\# 323110; RRID:AB_940461 \\
\hline Rabbit polyclonal to CRB3 & Szymaniak et al., 2015 & N/A \\
\hline Mouse IgG1 isotype, PE conjugated & BioLegend & Cat\# 400113; RRID:AB_326435 \\
\hline Mouse IgG1 isotype, PerCP-Cy5.5 conjugated & BioLegend & Cat\# 400149; RRID:AB_893680 \\
\hline Mouse monoclonal to E-Cadherin/CDH1 & BD Biosciences & Cat\# 610181; RRID:AB_397580 \\
\hline Mouse monoclonal to EPCAM & Santa Cruz & Cat\# Sc-73491; RRID:AB_1122569 \\
\hline Mouse monoclonal to GAPDH & Chemicon & Cat\# MAB374; RRID:AB_2107445 \\
\hline Rabbit monoclonal to NKX2-1 (clone EP15847) & Abcam & Cat\# ab76013; RRID:AB_1310784 \\
\hline Rabbit monoclonal to Phospho-Ezrin/Radixin/Moesin ( $p$-ERM) & Cell Signaling & Cat\# 3726; RRID:AB_10560513 \\
\hline Mouse monoclonal to pro-SFTPC & Santa Cruz & Cat\# sc-518029 \\
\hline Rabbit polyclonal to pro-SFTPC & Seven Hills & Cat\# WRAB-9337; RRID:AB_2335890 \\
\hline Rabbit polyclonal to mature SFTPC & Seven Hills & Cat\# WRAB-76694 \\
\hline Rabbit polyclonal anti-NPRO-SFTPC & Beers et al., 1994 & N/A \\
\hline Rabbit polyclonal anti-mature SFTPB (PT3) & Beers et al., 1992 & $\mathrm{~N} / \mathrm{A}$ \\
\hline Rabbit monoclonal to MUC1 & Abcam & Cat\# Ab218998 \\
\hline Rabbit polyclonal to HA tag & Abcam & Cat\# Ab20084; RRID:AB_445319 \\
\hline Mouse monoclonal to RAGE & Abcam & Cat\# Ab54741; RRID:AB_2242462 \\
\hline Rabbit polyclonal to LC3B & Cell Signaling & Cat\# 2775; RRID:AB_915950 \\
\hline Mouse monoclonal to SQSTM1/p62 & Abcam & Cat\# Ab56416; RRID:AB_945626 \\
\hline Mouse monoclonal to CPM & Wako & Cat\# 014-27501; RRID:AB_2801482 \\
\hline Mouse monoclonal to SLC34A2 & Yin et al., 2008 & $\mathrm{~N} / \mathrm{A}$ \\
\hline $\begin{array}{l}\text { AffiniPure Donkey Anti-Rabbit lgG }(\mathrm{H}+\mathrm{L}) \text {, AlexaFluor } \\
488 \text { conjugated }\end{array}$ & Jackson ImmunoResearch & Cat\# 711-545-152; RRID:AB_2313584 \\
\hline $\begin{array}{l}\text { AffiniPure Donkey Anti-Rabbit IgG }(\mathrm{H}+\mathrm{L}) \text {, AlexaFluor } \\
647 \text { conjugated }\end{array}$ & Jackson ImmunoResearch & Cat\# 711-605-152; RRID:AB_2492288 \\
\hline $\begin{array}{l}\text { AffiniPure Donkey Anti-Mouse } \lg G(H+L) \text {, AlexaFluor } \\
488 \text { conjugated }\end{array}$ & Jackson ImmunoResearch & Cat\# 715-545-150; RRID:AB_2340846 \\
\hline $\begin{array}{l}\text { AffiniPure Donkey Anti-Mouse IgG }(\mathrm{H}+\mathrm{L}) \text {, AlexaFluor } \\
647 \text { conjugated }\end{array}$ & Jackson ImmunoResearch & Cat\# 715-605-150; RRID:AB_2340862 \\
\hline $\begin{array}{l}\text { AffiniPure Goat Anti-Mouse IgG }(\mathrm{H}+\mathrm{L}) \text {, AlexaFluor } \\
488 \text { conjugated }\end{array}$ & Jackson ImmunoResearch & Cat\# 115-545-062; RRID:AB_2338845 \\
\hline $\begin{array}{l}\text { AffiniPure Goat Anti-Rabbit IgG }(H+L) \text {, AlexaFluor } \\
488 \text { conjugated }\end{array}$ & Jackson ImmunoResearch & Cat\# 111-545-144; RRID:AB_2338052 \\
\hline $\begin{array}{l}\text { AffiniPure Goat Anti-Mouse lgG }(H+L) \text {, AlexaFluor } \\
594 \text { conjugated }\end{array}$ & Jackson ImmunoResearch & Cat\# 115-585-146; RRID:AB_2338881 \\
\hline $\begin{array}{l}\text { AffiniPure Goat Anti-Rabbit lgG }(H+L) \text {, AlexaFluor } \\
594 \text { conjugated }\end{array}$ & Jackson ImmunoResearch & Cat\# 111-585-144; RRID:AB_2307325 \\
\hline Goat Anti-Mouse IgG $(\mathrm{H}+\mathrm{L}), \mathrm{HRP}$ conjugated & BioRad & Cat\# 0300-0108P; RRID:AB_808614 \\
\hline Goat Anti-Rabbit IgG $(\mathrm{H}+\mathrm{L})$, HRP conjugated & BioRad & Cat\# 5196-2504; RRID:AB_619908 \\
\hline
\end{tabular}




\begin{tabular}{|c|c|c|}
\hline Continued & & \\
\hline REAGENT or RESOURCE & SOURCE & IDENTIFIER \\
\hline Chemicals, peptides and recombinant proteins & & \\
\hline Human embryonic stem cell ( $\mathrm{hESC}$ )- qualified (2D) Matrigel & Corning & Cat\# 354277 \\
\hline Growth Factor Reduced (3D) Matrigel & Corning & Cat\# 356231 \\
\hline SB431542 & Tocris & Cat\# 1614 \\
\hline Dorsomorphin & Stemgent & Cat\# 04-0024 \\
\hline CHIR99021 (CHIR) & Tocris & Cat\# 4423 \\
\hline Recombinant human FGF10 & R\&D Systems & Cat\# 345-FG-025 \\
\hline Recombinant Human Keratinocyte Growth Factor (KGF) & R\&D Systems & Cat\# 251-KG-010 \\
\hline Recombinant Human BMP4 (rhBMP4) & R\&D Systems & Cat\# 314-BP \\
\hline Retinoic acid & Sigma Aldrich & Cat\# R2625 \\
\hline Y-27632 dihydrochloride & Tocris & Cat\# 1254 \\
\hline Ascorbic Acid & Sigma Aldrich & Cat\# A4544 \\
\hline 1-Thioglycerol (MTG) & Sigma Aldrich & Cat\# M6145 \\
\hline BSA $7.5 \%$ Stock & Thermo Fisher Scientific & Cat\# 15260037 \\
\hline 4-(2-hydroxyethyl)-1-piperazineethanesulfonic acid (EDTA) & Sigma Aldrich & Cat\# E7889 \\
\hline $\begin{array}{l}\text { N-(2-Hydroxyethyl)piperazine-N'-(2- ethanesulfonic acid) } \\
\text { Solution (HEPES) }\end{array}$ & Sigma Aldrich & Cat\# H0887 \\
\hline Dexamethasone & Sigma Aldrich & Cat\# D4902 \\
\hline $\begin{array}{l}\text { 8-bromoadenosine } 30,50 \text {-cyclic monophosphate sodium } \\
\text { salt (cAMP) }\end{array}$ & Sigma Aldrich & Cat\# B7880 \\
\hline 3-Isobutyl-1-methylxanthine (IBMX) & Sigma Aldrich & Cat\# 15879 \\
\hline Puromycin Dihydrochloride & Thermo Fisher Scientific & Cat\# A1113802 \\
\hline Geneticin Sulfate & Life Technologies & Cat\# 11811-023 \\
\hline $0.05 \%$ trypsin-EDTA & Invitrogen & Cat\# 25300-120 \\
\hline Defined Fetal Bovine Serum & Thermo Fisher Scientific & Cat\# NC0652331 \\
\hline Dimethyl Sulfoxide (DMSO) & Sigma Aldrich & Cat\# D2650 \\
\hline Calcein blue & Life Technologies & Cat\# C1429 \\
\hline Hoechst 33342 & Thermo Fisher Scientific & Cat\#H3570 \\
\hline DAPI, FluoroPure grade & Invitrogen & D21490 \\
\hline ProLong Diamond Antifade Mountant & Invitrogen & Cat\# P36965 \\
\hline LysoTracker Green DND-26 & Thermo Fisher Scientific & Cat\# L7526 \\
\hline MitoTracker Green FM & Thermo Fisher Scientific & Cat\# M7514 \\
\hline BODIPY-493/503 & Thermo Fisher Scientific & Cat\# D3922 \\
\hline LysoSensor Yellow/Blue Dextran & Thermo Fisher Scientific & Cat\# L22460 \\
\hline Dispase II & Thermo Fisher Scientific & Cat\# 17105-041 \\
\hline Glutaraldehyde & Ladd Research & Cat\# 20100 \\
\hline Osmium Tetroxide & Polysciences & Cat\# 0223D \\
\hline Uranyl Acetate & Electron Microscopy Sciences & Cat\# 22400 \\
\hline EMbed 812 & Electron Microscopy Sciences & Cat\# 14120 \\
\hline Paraformaldehyde & Electron Microscopy Sciences & Cat\# 19208 \\
\hline Oligomycin & Millipore & Cat\# 495455 \\
\hline FCCP & Sigma Aldrich & Cat\# C2920 \\
\hline Antimycin A & Sigma Aldrich & Cat\# A8674 \\
\hline Bafilomycin A1 & Sigma Aldrich & Cat\# B1793 \\
\hline Torin & Tocris & Cat\# 4247 \\
\hline Critical commercial assays & & \\
\hline RNeasy Mini Kit & QIAGEN & Cat\# 74104 \\
\hline QIAzol Lysis Reagent QIAGEN & QIAGEN & Cat\# 79306 \\
\hline
\end{tabular}




\begin{tabular}{|c|c|c|}
\hline Continued & & \\
\hline REAGENT or RESOURCE & SOURCE & IDENTIFIER \\
\hline TaqMan Fast Universal PCR Master Mix (2X) & Thermo Fisher & Cat\# 4364103 \\
\hline High-Capacity cDNA Reverse Transcription Kit & Applied Biosystems & Cat\# 4368814 \\
\hline EZ-TAL TALE Assembly Kit & System Bioscience & Cat\# GE120A-1 \\
\hline Click-iT EdU Alexa Fluor 647 Flow Cytometry Assay Kit & Thermo Fisher Scientific & Cat\# C10424 \\
\hline Lipofectamine LTX Kit & Thermo Fisher Scientific & Cat\# 15338100 \\
\hline P3 Primary Cell 4D-NucleofectorO X Kit S & Lonza & Cat\# V4XP-3032 \\
\hline $\begin{array}{l}\text { Magnetic Luminex } \circledast \text { Performance Assay Human } \\
\text { HighSensitivity }\end{array}$ & R\&D Systems & Cat\# LXSAHM-31 \\
\hline \multicolumn{3}{|l|}{ Deposited data } \\
\hline $\begin{array}{l}\text { Single cell transcriptomic profiling of patient-specific } \\
\text { pluripotent stem cell-derived alveolar epithelial type } \\
2 \text { cells expressing the SFTPC }{ }^{\prime 73 T} \text { variant and their } \\
\text { gene-edited corrected counterparts }\end{array}$ & This paper & GEO (GSE160801) \\
\hline $\begin{array}{l}\text { Proteomic and phosphoproteomic profiling of SFTPC }{ }^{173 T} \\
\text { expressing patient-specific pluripotent stem cell-derived } \\
\text { alveolar epithelial type } 2 \text { cells and their gene-edited } \\
\text { corrected counterparts. }\end{array}$ & This paper & PRIDE: PXD022334 \\
\hline \multicolumn{3}{|l|}{ Experimental models: Cell lines } \\
\hline Human: SFTPC ${ }^{173 T / W T}$ donor iPSC line (SPC2) & This paper & $\begin{array}{l}\text { http://stemcellbank.bu.edu/ } \\
\text { Catalog/ltem/Home }\end{array}$ \\
\hline $\begin{array}{l}\text { Human: Corrected SFTPC donor iPSC line } \\
\text { (SPC2-CorrC1; SFTPC }\end{array}$ & This paper & $\begin{array}{l}\text { http://stemcellbank.bu.edu/ } \\
\text { Catalog/ltem/Home }\end{array}$ \\
\hline $\begin{array}{l}\text { Human: Donor iPSC line targeted with } \\
\text { SFTPC }^{\text {tdTomato }} \text { (SPC2-ST-C11; SFTPC }\end{array}$ & This paper & $\begin{array}{l}\text { http://stemcellbank.bu.edu/ } \\
\text { Catalog/Item/Home }\end{array}$ \\
\hline $\begin{array}{l}\text { Human: Donor iPSC line targeted with } \\
\text { SFTPC }^{\text {tdTomato }} \text { (SPC2-ST-B2; SFTPC }{ }^{\text {tdT/WT }} \text { ) }\end{array}$ & Kotton Lab; Hurley et al., 2020 & $\begin{array}{l}\text { http://stemcellbank.bu.edu/ } \\
\text { Catalog/ltem/Home }\end{array}$ \\
\hline \multicolumn{3}{|l|}{ Oligonucleotides } \\
\hline See Table S3 & This paper & N/A \\
\hline \multicolumn{3}{|l|}{ Recombinant DNA } \\
\hline $\begin{array}{l}\text { EF1a-TALEN_HD; SFTPC recognition sequence of } \\
\text { right TALEN 5'-TCA CCG GCG GGC TCT CCA TC-3'. }\end{array}$ & Kotton Lab; Jacob et al., 2017 & http://www.kottonlab.com \\
\hline $\begin{array}{l}\text { EF1a-TALEN_NN; SFTPC recognition sequence of } \\
\text { left TALEN: 5'-TAG CAC CTG CAG CAA GAT GG-3'. }\end{array}$ & Kotton Lab; Jacob et al., 2017 & http://www.kottonlab.com \\
\hline p1303-DV-SFTPC-tdTomato & Kotton Lab; Jacob et al., 2017 & http://www.kottonlab.com \\
\hline pHAGE2 EF1a-Cre-IRES-NeoR-W & Kotton Lab; Jacob et al., 2017 & http://www.kottonlab.com \\
\hline pHAGE2-Cre-IRES-PuroR & Kotton Lab; Somers et al., 2010 & Addgene \#30205 \\
\hline \multicolumn{3}{|l|}{ Software and algorithms } \\
\hline FlowJo version 10.5 .3 & Becton, Dickinson \& Company & https://www.flowjo.com/ \\
\hline ImageJ & National Institutes of Health & https://imagej.nih.gov/ij/ \\
\hline CellProfiler v2.0 & Broad Institute & https://cellprofiler.org \\
\hline Prism & GraphPad & https://www.graphpad.com/ \\
\hline Original code & This paper & $\begin{array}{l}\text { https://github.com/CReM- } \\
\text { BU/Alysandratos_iAEC2s }\end{array}$ \\
\hline \multicolumn{3}{|l|}{ Other } \\
\hline StemDiff Definitive Endoderm Kit & StemCell Technologies & Cat\# 05110 \\
\hline mTeSR1 & StemCell Technologies & Cat\# 05850 \\
\hline Glutamax & Life Technologies & Cat\# 35050-061 \\
\hline Gentle Cell Dissociation Reagent & StemCell Technologies & Cat\# 07174 \\
\hline Seahorse XF Base Medium Minimal DMEM & Agilent Technologies & Cat\# 103193-100 \\
\hline
\end{tabular}

(Continued on next page) 


\begin{tabular}{|c|c|c|}
\hline \multicolumn{3}{|l|}{ Continued } \\
\hline REAGENT or RESOURCE & SOURCE & IDENTIFIER \\
\hline $\begin{array}{l}\text { Hank's Buffered Saline Solution } \\
\text { (HBSS; no calcium, no magnesium, no phenol red) }\end{array}$ & GIBCO & Cat\# 14175095 \\
\hline Ham's F12 Medium & Cellgro & Cat\# 10-080-CV \\
\hline Iscove's Modified Dulbecco's Medium (IMDM) & Thermo Fisher Scientific & Cat\# 12440053 \\
\hline N2 Supplement & Invitrogen & Cat\# 17502-048 \\
\hline B27 Supplement & Invitrogen & Cat\# 15260-037 \\
\hline Primocin & Invitrogen & Cat\# NC9141851 \\
\hline
\end{tabular}

\section{RESOURCE AVAILABILITY}

\section{Lead contact}

Further information and requests for reagents may be directed to, and will be fulfilled by, the Lead Contact, Darrell Kotton (dkotton@bu.edu).

\section{Materials availability}

All unique/stable reagents generated in this study are available from the Lead Contact with a completed Materials Transfer Agreement. Pluripotent stem cell lines generated in this study are available from the CReM iPSC Repository at Boston University and Boston Medical Center and can be found at https://www.bu.edu:443/dbin/stemcells/.

\section{Data and code availability}

The scRNA-seq data reported in this publication have been deposited in NCBI's Gene Expression Omnibus (GEO; accession number GSE160801). The mass spectrometry proteomics data have been deposited to the ProteomeXchange Consortium via the Proteomics Identifications (PRIDE) partner repository with the dataset identifier PRIDE: PXD022334. All original code has been deposited at GitHub and is publicly available as of the date of publication. DOls are listed in the key resources table. Any additional information required to reanalyze the data reported in this paper is available from the lead contact upon request.

\section{EXPERIMENTAL MODEL AND SUBJECT DETAILS}

\section{iPSC line generation and maintenance}

All experiments involving the differentiation of human iPSC lines were performed with the approval of the Institutional Review Board of Boston University (protocol H33122). The Human Research Protection Office of Washington University School of Medicine, St. Louis, MO, approved procurement of dermal fibroblasts for reprogramming with written informed consent. The iPSC line SPC2 was derived by reprogramming these fibroblasts from a patient with childhood interstitial lung disease (chILD) that progressed to end stage lung disease with pulmonary fibrosis requiring lung transplantation (clinical details provided in the supplemental data). Gene sequencing documented a heterozygous SFTPC pathogenic variant in the non-BRICHOS domain, c.218 T > C (p.I73T) (Figure 1). Reprogramming to generate iPSCs was performed using the excisable, floxed lentiviral STEMCCA vector followed by excision of the single integrated lentiviral copy via transient Cre-recombinase exposure and confirmation of successful STEMCCA excision (SPC2-18Cr1 clone; SFTPC ${ }^{173 T / W T}$ ) prior to directed differentiation, as previously published (Somers et al., 2010). We have previously published partial characterization of the corrected SPC2 line (SPC2-ST-B2), without disease modeling, including stability of the AEC2 phenotype in the corrected iAEC2s derived from this clone after prolonged cell culture expansion (Hurley et al., 2020). The SPC6 and SPC7 iPSC lines were generated by reprogramming peripheral blood mononuclear cells (PBMCs) from two individuals with the SFTPC ${ }^{173 T}$ variant and clinically phenotyped chILD (clinical details provided in the supplemental data) using the CytoTune-iPS 2.0 Sendai Reprogramming Kit (Invitrogen, Thermofisher, Cat\# A16517) according to the manufacturer's instructions, with detailed methods available for free download from the CReM's protocols page at https://www. bumc.bu.edu/stemcells. The Institutional Review Board of Boston University approved procurement of these PBMCs and reprogramming into iPSCs with written informed consent.

All iPSC lines (SPC2-ST-B2, SPC2-ST-C11, SPC2-18Cr1, SPC2-CorrC1, SPC6, and SPC7) used in this study demonstrated a normal karyotype (46XY or 46XX) when analyzed by G-banding and/or array Comparative Genomic Hybridization (aCGH) both before and after gene-editing (Cell Line Genetics) (Figure S6A). Pluripotency was confirmed by staining for pluripotency markers (Figure S6B) as previously described (Somers et al., 2010). Culture conditions used for maintenance and editing of undifferentiated iPSCs were as follows: for Transcription activator-like effector nucleases (TALEN) targeting, iPSC lines were maintained on mitomycin C-inactivated mouse embryonic fibroblasts (MEFs) in human iPSC media (WiCell feeder dependent protocol). For CRISPR targeting and prior to directed differentiation, all iPSC lines were maintained in feeder-free conditions, on growth factor reduced Matrigel (Corning) in 6-well tissue culture dishes (Corning), in mTeSR1 media (StemCell Technologies) using gentle cell dissociation reagent (GCDR) 
for passaging. Further details of iPSC derivation, characterization, and culture are available for free download at https://www.bu. edu:443/dbin/stemcells/protocols.php.

\section{Generation of gene-edited syngeneic iPSC lines}

To generate gene-edited corrected syngeneic iPSC lines two approaches were undertaken. In the first approach, we used TALENs to insert a tdTomato fluorescent reporter at the translation initiation (ATG) site of the endogenous SFTPC locus of the parental SPC2 iPSC line, as previously described (Jacob et al., 2017). All TALENs and donor template plasmid maps and sequences are available at www.kottonlab.com. The SFTPC TALEN recognition sequences are: left TALEN 5'-TAG CAC CTG CAG CAA GAT GG-3' and right TALEN 5'-TCA CCG GCG GGC TCT CCA TC-3'. Between the two binding sites is a 22 bp spacer (ATG TGG GCA GCA AAG AGG TCC T). TALENs were constructed using EZ-TAL TALE Assembly Kit (System Bioscience), according to manufacturer's instructions, and the resulting SFTPC TALENs encoding plasmids were named EF1a-TALEN_NN (SPC left) and EF1a-TALEN_HD (SPC right), respectively.

To deliver the donor template to the SFTPC locus, we generated a donor vector (p1303 DV-SFTPC-tdTomato) containing the tdTomato coding sequence and a floxed PGK promoter-driven puromycin resistance cassette, flanked by left and right arms of homology to the human endogenous SFTPC locus, as previously described (Jacob et al., 2017). The TALENs and donor vector plasmids were co-transfected into the SPC2 iPSC line using a lipofectamine based transfection protocol. For this purpose, SPC2 iPSCs were plated onto a mitomycin C-inactivated DR4 MEF feeder layer and cultured in human iPSC media (WiCell) in a 6-well plate. After the cells reached $50 \%$ confluence, they were transfected with the two TALENs and tdTomato donor vector as follows: $3 \mu \mathrm{g}$ of donor vector and $1.2 \mu \mathrm{g}$ of each TALEN were added to $275 \mu \mathrm{l}$ of IMDM and $4 \mu \mathrm{l}$ of Plus reagent from the Lipofectamine LTX kit (Thermo Fisher Scientific) and the mixture was incubated at room temperature for 5 minutes. $16 \mu$ l of lipofectamine LTX from the same kit was added to another $275 \mu$ l of IMDM. $275 \mu \mathrm{L}$ of the DNA mixture was added to $275 \mu \mathrm{L}$ of the LTX mixture and incubated at room temperature for 30 minutes. $550 \mu \mathrm{l}$ of the total mixture was added drop by drop to 1 well of a 6-well plate. Five hours later, the media was changed, and 48 hours later, $0.7 \mu \mathrm{g} / \mathrm{ml}$ puromycin (Thermo Fisher Scientific) was added to the media for 4 days to select antibiotic resistant colonies. After 10 days individual colonies were picked and screened for targeting using the following primer pairs: $5^{\prime}$ GGG TGA GTG AGC TGA TTC GAG 3', 5' TGA CCT CCT CGC CCT TGC TCA CCA TG 3'. DNA sequencing using a primer binding in the genome outside any regions included in targeting plasmids confirmed targeting into the endogenous SFTPC locus (5' GGG TGA GTG AGC TGA TTC GAG $3^{\prime}$ ). To confirm heterozygous targeting, colonies were screened for a remaining intact SFTPC allele using the following primers: 5' CTA CGG ACA CAT ATA AGA CCC TGG TC 3', 5' CAA ATA GCC ACC ATT CGG ACA CTC 3'. DNA sequencing of the amplified portion of the intact SFTPC allele was performed using the following primer $5^{\prime}$ TAT GGG GAT GGG TAC CAC TGG CTG $3^{\prime}$ to pick colonies of either corrected (SPC2-ST-B2 clone; SFTPC ${ }^{\text {tdT/WT }}$ ) or mutant (SPC2-ST-C11 clone; SFTPC ${ }^{173 T / t d T}$ ) iPSC clones as the tdTomato cassette is followed by a stop/polyA cassette, preventing expression of the subsequent SFTPC coding sequence from the targeted allele (Figure 1D).

Cre-mediated excision of the floxed puromycin resistance cassette was performed using a plasmid containing Cre-recombinase and neomycin resistance (PHAGE2 EF1a-Cre-IRES-NeoR-W; www.kottonlab.com) using the same lipofectamine-based protocol described above, with 4 days of $200 \mathrm{ng} / \mu$ l geneticin-based (Life Tech) selection for clones that were transfected with Cre-containing plasmid. Excision of the puromycin cassette was confirmed by PCR using the following primers: $5^{\prime}$ ATG ACC GAG TAC AAG CCC ACG 3', 5' TCA GGC ACC GGG CTT GC 3'.

In the second approach, we employed the CRISRP/Cas9 gene editing technology to generate a gene-edited corrected iPSC line that expressed two active SFTPC alleles (SPC2-CorrC1 clone; SFTPC ${ }^{\text {WT/WT }}$ ) as described below. Using the CRISPR/Cas9 technology we targeted the region adjacent to the I73T mutation (c.218 T > C (p.I73T)) in the SFTPC gene locus (Figure S1B) with a guide RNA (specifically recognizing the 173T mutation but not wild-type) that had the following sequence: 5' CAG GTT CTG GAG ATG AGC AC 3'. gRNA sequences were cloned into the pD1321-AD plasmid backbone (DNA 2.0 ATUM) that contains a M-dasher-GFP sequence fused to Cas9. Delivery of this plasmid to iPSCs enabled co-expression of Cas9, gRNA, and a GFP reporter. To accomplish footprint-free correction of the SFTPC variant, a short single stranded DNA oligo (sequence 5' GGT GGC TCC ATG CCC TTT CCC CAG GTT CTG GAA ATG TCA ATT GGT GCG CCG GAA GCC CAG CAA CGC CTG GCC CTG AGT GAG 3') was used as a donor template containing the wild-type SFTPC sequence. SPC2 cells maintained in feeder-free conditions in mTeSR 1 media were treated with $10 \mu \mathrm{M}$ Y-27632 (Tocris) for 3 hours, dissociated in GCDR (StemCell Technologies) for 10 minutes at $37^{\circ} \mathrm{C}$ and counted using a Luna-II Automated Cell Counter (Logos Biosystems, Annandale, VA). Approximately $5 \times 10^{6}$ cells were centrifuged at $200 \mathrm{~g}$ for 5 minutes, resuspended in a mixture containing P3 solution and supplement (P3 Primary Cell 4D-Nucleofector X Kit L, Lonza) as well as $5 \mu \mathrm{g}$ CRISPR/Cas9 plasmid and $5 \mu \mathrm{g}$ oligo donor, and nucleofected using program code CB-150 in the 4D Nucleofector X System (Lonza). Nucleofected cells were replated on 4 wells of a Matrigel-coated 6-well plate in mTeSR1 media, and $10 \mu \mathrm{M}$ Y-27632 was added for 24 hours. After 48 hours, cells were prepared for sorting. They were treated with $10 \mu \mathrm{M} \mathrm{Y-27632} \mathrm{for} 3$ hours, dissociated into single cell suspension with GCDR for $10-15$ minutes at $37^{\circ} \mathrm{C}$, centrifuged at $200 \mathrm{~g}$ for 5 minutes, resuspended in mTeSR1 media supplemented with $10 \mu \mathrm{M}$ Y-27632, and filtered through a $30 \mu \mathrm{m}$ filter (Falcon). GFP+ cells were sorted into recovery media (1 part mTeSR1 and 1 part conditioned mTeSR1 supplemented with $0.7 \mathrm{ng} / \mathrm{ml} \mathrm{FGF2,} \mathrm{plus} 10 \mu \mathrm{M}$ Y-27632) using a high-speed cell sorter (MoFlo Legacy, Beckman Coulter). 1×104 GFP+ sorted cells were plated into a $10 \mathrm{~cm}$ tissue culture treated dish pre-coated with growth factor reduced Matrigel (Corning). Recovery media was changed every other day for the first 5 days, with $10 \mu \mathrm{M} \mathrm{Y-27632}$ added for the first 24 hours. After 5 days, mTeSR 1 was used to feed the cells daily, and after 10 days, colonies that emerged were of 
sufficient size for picking for clonal expansion and screening. Individual colonies were picked and screened for correction using the following primers: 5' GTC GTG GTG ATT GTG GGA GCC CTG 3', 5' CTG GGC ATA CCC ACC TGC TGG TAG 3’. DNA sequencing using the following primer $5^{\prime}$ TAT GGG GAT GGG TAC CAC TGG CTG $3^{\prime}$ confirmed correction of the SFTPC variant, resulting in generation of the SPC2-Corr line.

\section{METHOD DETAILS}

iPSC directed differentiation into alveolar epithelial type 2 cells (iAEC2s)

To generate iAEC2s, we performed PSC directed differentiation via definitive endoderm into NKX2-1 lung progenitors using methods we have previously described (Hawkins et al., 2017; Jacob et al., 2017, 2019). In brief, cells maintained in mTeSR1 media were differentiated into definitive endoderm using the STEMdiff Definitive Endoderm Kit (StemCell Technologies) for 3 days (day 0-3) and after the endoderm-induction stage, cells were dissociated with GCDR and passaged into 6-well dishes pre-coated with growth factor reduced Matrigel in "DS/SB" anteriorization media, consisting of complete serum-free differentiation media (cSFDM) base as previously described (Jacob et al., 2017, 2019), supplemented with $2 \mu$ M Dorsomorphin ("DS"; Stemgent) and $10 \mu$ M SB431542 ("SB"; Tocris). For the first $24 \mathrm{hr}$ after passaging, $10 \mu \mathrm{M}$ Y-27632 was added to the media. After anteriorization in "DS/SB" media for 3 days (day 3-6), cells were cultured in "CBRa" lung progenitor-induction media until day 15 or 16, as we have previously published (Jacob et al., 2017; Serra et al., 2017). "CBRa”' media consists of cSFDM base supplemented with $3 \mu$ M CHIR99021 (Tocris), $10 \mathrm{ng} / \mathrm{ml}$ recombinant human BMP4 (rhBMP4, R\&D Systems), and $100 \mathrm{nM}$ retinoic acid (RA, Sigma). On day 15 or 16 of differentiation, live cells were sorted on a high-speed cell sorter (MoFlo Legacy or MoFlo Astrios EQ) to isolate NKX2-1+ lung progenitors based on CD47 hi/ $\mathrm{CD} 6^{\text {neg }}$ gating (Hawkins et al., 2017). Sorted lung progenitors were resuspended in undiluted growth factor reduced 3D Matrigel (Corning) at a concentration of approximately $400 \mathrm{cells} / \mu \mathrm{l}$ and distal/alveolar differentiation of cells was performed in "CK+DCl" media, consisting of cSFDM base supplemented with $3 \mu \mathrm{M}$ CHIR99021, $10 \mathrm{ng} / \mathrm{mL}$ rhKGF (CK), and $50 \mathrm{nM}$ dexamethasone (Sigma), $0.1 \mathrm{mM}$ 8-Bromoadenosine 30,50-cyclic monophosphate sodium salt (Sigma) and 0.1 mM 3-Isobutyl-1-methylxanthine (IBMX; Sigma) (DCl). The resulting epithelial spheres were passaged without further sorting on approximate day 30 (day 28-37) of differentiation and a brief period (4-5 days) of CHIR99021 withdrawal followed by one week of CHIR99021 addback was performed (Figure 1F) to achieve iAEC2 maturation, as previously shown (Jacob et al., 2017). After this 14-day period, SFTPC ${ }^{\text {tdTomato+ }}$ cells were purified by fluorescence activated cell sorting (FACS) and resuspended in undiluted growth factor reduced 3D Matrigel (Corning) at a concentration of approximately $400 \mathrm{cells} / \mu \mathrm{l}$ in "CK+DCl" media to establish pure long-term iAEC2s cultures. iAEC2s were then maintained through serial passaging as self-renewing monolayered epithelial spheres ("alveolospheres") by plating in 3D Matrigel (Corning) droplets at a density of 400 cells/ $\mu$ l with refeeding every other day with "CK+DCl" media, according to our previously published protocol (Jacob et al., 2019). iAEC2 culture quality and purity was monitored at each passage by flow cytometry, with $>95 \%$ of cells expressing SFTPC ${ }^{\text {tdTomato }}$ over time (Figure $2 \mathrm{~A}$ ).

\section{Murine model of Sftpc ${ }^{173 T}$}

Tamoxifen inducible Sftpc ${ }^{I 73 T}$ mice were generated and induced following previously reported protocols (Katzen et al., 2019; Nureki et al., 2018). Briefly, the Sftpc ${ }^{173 T}$ founder line, expressing a neomycin cassette, was crossed with a mouse line expressing an estrogen receptor (ER)-2 controlled Flp-O recombinase strain knocked into the Rosa26 locus (Jackson Laboratory, Bar Harbor, ME) to generate the inducible-Sftpc $c^{173 T F l p}$ line. Adult homozygote Sftpc $c^{173 T F / p}$ mice received tamoxifen $(260 \mathrm{or} 360 \mathrm{mg} / \mathrm{kg}$ in corn oil, oral gavage) at 12-14 weeks of age. AEC2 isolation was performed 14 days after tamoxifen induction.

\section{Mouse AEC2 isolation}

Mouse AEC2s were isolated as previously reported (Atochina-Vasserman et al., 2011). Briefly, perfused mouse lungs were digested with dispase (BD Biosciences), mechanically dissociated, and treated with $25 \mu \mathrm{g} / \mathrm{ml}$ DNAase I (Sigma-Aldrich). Negative selection of mesenchymal cells (fibroblasts) was done by differential adherence on plastic culture dishes. CD45-positive cells were removed by negative selection using Dynabeads Untouched Mouse T Cells Kit (11413D) and Dynabeads Mouse DC Enrichment Kit (11429D; Thermo Fisher Scientific).

\section{Flow cytometry and Fluorescence Activated Cell Sorting (FACS)}

Preparation of single cell suspensions of 3D Matrigel-embedded iAEC2s for flow cytometry and FACS was achieved by incubation with $2 \mathrm{mg} / \mathrm{ml}$ dispase (Thermo Fisher Scientific) for $30-60$ minutes at $37^{\circ} \mathrm{C}$ and subsequent incubation with $0.05 \%$ trypsin for $12-15$ minutes at $37^{\circ} \mathrm{C}$, as previously described (Jacob et al., 2019). Cells were washed with media containing $10 \%$ fetal bovine serum (FBS, Thermo Fisher Scientific). Harvested cells were centrifuged at $300 \mathrm{~g}$ for 5 minutes at $4^{\circ} \mathrm{C}$ and resuspended in FACS buffer containing Hank's Balanced Salt Solution (Thermo Fisher Scientific), 2\% FBS and $10 \mu \mathrm{M}$ Y-27632 (Tocris) and stained with calcein blue AM (Thermo Fisher Scientific) for dead cell exclusion during flow cytometry. Live cells were sorted on a high-speed cell sorter (MoFlo Legacy or MoFlo Astrios EQ, Beckman Coulter, Pasadena, CA) at the Boston University Medical Center Flow Cytometry Core Facility.

Immunostaining of cells in single cell suspension with antibodies against CD47 (Biolegend) and CD26 (Biolegend) and FACS gating of the CD47 $1 / C D 26^{\text {neg }}$ population for purification of lung progenitors was performed as previously described (Hawkins et al., 2017; 
Jacob et al., 2019). Purification of iAEC2s for the establishment of pure long-term iAEC2 cultures following iAEC2 maturation (via CHIR99021 withdrawal and addback) in the iPSC lines that had not been targeted with any fluorochrome reporters was achieved by staining for carboxypeptidase M (CPM) (SPC2-18Cr1 and SPC2-CorrC1) or SLC34A2 (SPC6 and SPC7) as detailed in Jacob et al. (2019): single cell suspicions were stained on ice in FACS buffer using a primary mouse monoclonal antibody against human CPM (1:200, Fujifilm Wako) or SLC34A2 (MX35 1:200, a generous gift of Dr. Gerd Ritter, Memorial Sloan Kettering) for 30 minutes and subsequently stained with secondary Alexa Fluor 647 conjugated antibody (1:500, Thermo Fisher Scientific) for 20 minutes.

Gating was based on isotype-stained controls or in the case of SFTPC ${ }^{\text {tdTomato }}$ on non-lung endoderm outgrowths (day 15-16 sorted CD47 ${ }^{\mathrm{lo}}$ cells). Flow cytometry staining was quantified using the Stratedigm S1000EXI and analyzed with FlowJo v10.6.2 (FlowJo, Tree Star Inc). Flow cytometry plots shown represent single cells after forward-scatter/side-scatter gating to remove debris as we have previously detailed in Jacob et al. (2019).

\section{Immunofluorescence microscopy}

Images of cultured 3D Matrigel-embedded iPSC-derived epithelial spheres were taken on a Keyence BZ-X700 fluorescence microscope (Osaka, Japan). Z stack images were processed using full focus image analysis using Keyence software.

For the analysis of lysosomal, mitochondrial, and lipid droplet morphology, alveolospheres were harvested by incubating with $2 \mathrm{mg} / \mathrm{ml}$ dispase (Thermo Fisher Scientific) for 30-60 minutes at $37^{\circ} \mathrm{C}$ and then stained with Hoechst $33432(1 \mu \mathrm{g} / \mathrm{ml}$, Thermo Fisher Scientific) and LysoTracker Green DND-26 (1 $\mu \mathrm{M}$, Thermo Fisher Scientific), MitoTracker Green (200 nM, Thermo Fisher Scientific), or BODIPY-493/503 $\left(1 \mu \mathrm{g} / \mathrm{ml}\right.$, Thermo Fisher Scientific) respectively for 45 minutes in $\mathrm{CK}+\mathrm{DCl}$ media at $37^{\circ} \mathrm{C}$ and $5 \% \mathrm{CO}_{2}$. After 2 washes with fresh media, alveolospheres were plated onto Greiner CELLview glass-bottom 4-compartment dishes (Greiner BioOne $\mathrm{GmbH}$ ) in 3D Matrigel (Corning) and CK+DCl media. Alveolospheres were imaged using a Zeiss LSM 880 confocal microscope (Carl Zeiss AG, Oberkochen) with Airyscan super resolution detector using a 40x or 63x oil objective. For all samples Hoechst fluorescence, green fluorescence (LysoTracker, MitoTracker, or BODIPY-493/503), as well as tdTomato fluorescence were recorded. For the duration of the imaging session alveolospheres were kept on a temperature and $\mathrm{CO}_{2}$ controlled humidified stage at $37^{\circ} \mathrm{C}$ and $5 \% \mathrm{CO}_{2}$.

For the analysis of lysosomal pH, alveolospheres were stained with LysoSensor Yellow/Blue Dextran (5 mg/ml, Thermo Fisher Scientific) for 1 hour in $\mathrm{CK}+\mathrm{DCl}$ media at $37^{\circ} \mathrm{C}$ and $5 \% \mathrm{CO}_{2}$. Subsequently, after 2 washes with fresh media alveolospheres were plated onto Greiner CELLview glass-bottom 4-compartment dishes in 3D Matrigel and CK+DCI media. After a 24-hour chase period alveolospheres were imaged using a Zeiss LSM 880 using 2-photon excitation at $720 \mathrm{~nm}$ and a $40 x$ oil objective. LysoSensor fluorescence was recorded as blue emission (401-461 nm) and yellow emission (511-550 nm). Additionally, tdTomato fluorescence was recorded using $561 \mathrm{~nm}$ laser excitation and 571-620 nm emission. For the duration of the imaging session alveolospheres were kept on a temperature and $\mathrm{CO}_{2}$ controlled humidified stage at $37^{\circ} \mathrm{C}$ and $5 \% \mathrm{CO}_{2}$. For the determination of $\mathrm{pH}$ standard curves, alveolospheres stained with LysoSensor Yellow/Blue Dextran were fixed in 4\% paraformaldehyde for 20 minutes. After 3 washes with PBS (pH 7.4), alveolospheres were equilibrated in MES buffers ( $\mathrm{pH}$ range between 4.5 and 6.0). LysoSensor fluorescence was recorded as above.

For formalin fixation and paraffin embedding, alveolospheres were harvested by incubating with $2 \mathrm{mg} / \mathrm{ml}$ dispase (Thermo Fisher Scientific) for $30-60$ minutes at $37^{\circ} \mathrm{C}$, fixed with fresh $4 \%$ paraformaldehyde overnight at $4^{\circ} \mathrm{C}$, combined in low melting agarose (SeaPrep) to form a pellet and then paraffin embedded. For immunocytochemistry, sections were rehydrated and antigen retrieval was performed at $95^{\circ} \mathrm{C}$ for 20 minutes in Dako Target Retrieval Solution (DAKO, S-1699). Sections were permeabilized with 0.5\% Triton X-100 (Sigma-Aldrich), blocked with 4\% normal donkey serum (NDS) for 1 hour, and incubated in primary antibody in $0.5 \%$ Triton X-100 and $4 \%$ NDS overnight at $4{ }^{\circ} \mathrm{C}$. The staining was detected with secondary Alexa Fluor-conjugated anti-lgG antibodies purchased from Jackson Immunoresearch (donkey anti-rabbit, goat anti-mouse, goat anti-rabbit at 1:400 to 1:500 dilution for 1 hour at room temperature) or Bio-Rad (goat anti-mouse, goat anti-rabbit at 1:10,000 dilution for 1 hour at room temperature) (see Key resources table), as previously described (Hawkins et al., 2015, 2017; Nureki et al., 2018). Nuclear counterstaining was performed with Hoechst dye (1:500, Invitrogen) or DAPI (300 nM, Invitrogen). Coverslips with cells were mounted on glass slides using Prolong Diamond Anti-Fade Mounting Reagent (Thermo Fisher Scientific) and imaged on an Olympus I-70 inverted fluorescent microscope (Olympus, Melville, NY), captured with a Hamamatsu 12-bit coupled-charged camera (Hamamatsu, Japan), and processed using Metamorph, Version 7.8.4.0 (Universal Imaging, West Chester, PA) or imaged using a Zeiss LSM 880 confocal microscope (Carl Zeiss AG, Oberkochen) and processed using ImageJ. Primary antibodies (see Key resources table) include: a polyclonal anti-pro-SFTPC antibody ("NPRO-SFTPC," 1:200) raised against the Met [10]-Glu [23] domain of rat pro-SFTPC and polyclonal anti-SFTPB ("PT3") raised against bovine SFTPB both produced in rabbits in house and validated as previously published (Beers et al., 1992, 1994; Beers and Lomax, 1995), as well as a rabbit polyclonal anti-CRB3 antibody (1:1000; Szymaniak et al., 2015). Other commercially obtained antibodies include: anti-activated caspase 3 (rabbit polyclonal, 1:200, Sigma-Aldrich), anti-E-Cadherin (mouse monoclonal, 1:500, BD Biosciences), anti-EPCAM (mouse monoclonal, 1:100, Santa Cruz), anti-HA (rabbit polyclonal, 1:40,000, Abcam), anti-p-ERM (rabbit monoclonal, 1:1000, Cell Signaling), anti-pro-SFTPC (rabbit polyclonal, 1:500, Seven Hills and mouse monoclonal, 1:500, Santa Cruz), anti-MUC1 (rabbit monoclonal, 1:100, Abcam), anti-RAGE (mouse monoclonal, 1:250, Abcam).

\section{Cell proliferation analysis}

To assess DNA replication as a method of proliferation analysis, 5-ethynyl-2'-deoxyuridine (EdU) incorporation was evaluated using the Click-iT EdU Alexa Fluor 647 Flow Cytometry Assay Kit (Invitrogen). Corrected and mutant SFTPC ${ }^{\text {tdTomato+ }}$ iAEC2s were plated at 
a concentration of 400 cells/ $\mu \mathrm{l}$ in 3D Matrigel in $\mathrm{CK}+\mathrm{DCl}$ media as described above. Seven days after plating, EdU was added to the cultures ( $n=3$ independent droplets) for 24 hours at a final concentration of $10 \mu \mathrm{M}$. The cells were then dissociated into single cell suspension as described above, fixed, permeabilized, and stained with the Click-iT reaction mixture according to the manufacturer's instructions. The percentage of SFTPC ${ }^{\text {tdTomato+ }}$ cells that incorporated EdU was determined by flow cytometry (Stratedigm).

\section{Transmission electron microscopy}

Intact alveolospheres were harvested by incubating with $2 \mathrm{mg} / \mathrm{ml}$ dispase (Thermo Fisher Scientific) for $30-60 \mathrm{minutes}$ at $37^{\circ} \mathrm{C}$ and fixed for 3 hours total in $2.5 \%$ glutaraldehyde (Ladd Research) in $0.1 \%$ cacodylate buffer (pH 7.4) at room temperature as follows: an equal volume of $5 \%$ glutaraldehyde/ $0.1 \mathrm{M}$ cacodylate was added to Eppendorf tubes containing the alveolospheres in known volume of $\mathrm{CK}+\mathrm{DCl}$ media, fixed for 1.5 hours, and centrifuged gently ( $300 \mathrm{~g}$ for 1 minute) followed by the addition of fresh $2.5 \%$ glutaraldehyde/ $0.1 \mathrm{M}$ cacodylate for an additional 1.5 hours at room temperature. The samples were then washed 3 times in $0.1 \mathrm{M}$ cacodylate, post-fixed in 1\% Tannic Acid in cacodylate buffer for 5 minutes at room temperature, washed again 3 times in cacodylate buffer, and post-fixed overnight in $1.5 \%$ osmium tetroxide (Polysciences) in $0.1 \mathrm{M}$ cacodylate buffer in dark at $4{ }^{\circ} \mathrm{C}$. The samples were then washed 3-4 times in $0.05 \mathrm{M} \mathrm{Na}$ Maleate buffer $(\mathrm{pH} 5.2)$ and block stained in 1.5\% Uranyl acetate (Electron Microscopy Sciences, EMS) in $0.025 \mathrm{M}$ Na Maleate buffer (pH 6.0). Next, the samples were dehydrated quickly through acetone on ice, from $70 \%$ to $80 \%$ to $90 \%$. The samples were then incubated 2 times in $100 \%$ acetone at room temperature for 10 minutes each, and in propylene oxide at room temperature for 15 minutes each. Finally, the samples were changed into EMbed 812 (EMS), left for 2 hours at room temperature, changed into fresh Embed 812 and left overnight at room temperature, after which they were embedded in fresh EMbed 812 and polymerized overnight at $60^{\circ} \mathrm{C}$. Plastic embedded samples were thin sectioned at $70 \mathrm{~nm}$ and grids were stained in $4 \%$ aqueous Uranyl Acetate for 5 minutes at $60^{\circ} \mathrm{C}$ followed by Lead Citrate for 10 minutes at room temperature. Electron microscopy was performed on a Philips CM12 EM operated at 100kV, and images were recorded on a TVIPS F216 CMOS camera with a pixel size of $0.85-3.80 \mathrm{~nm}$ per pixel.

\section{Other staining}

Toluidine blue staining was performed as follows: $0.5 \mu \mathrm{m}$ sections (from EMbed plastic blocks) were collected, dried for 30 minutes on a hot plate, and stained 30-60 s in 0.5\% Toluidine blue + 0.5\% Borax in dH20, rinsed in dH20, dried and coverslipped. For Hematoxylin and Eosin (H\&E) staining standard methods were used (AML Labs, Inc, Baltimore, MD).

\section{Quantification of autophagic flux}

To quantify autophagosomes, corrected (SFTPC ${ }^{\text {tdT/WT }}$ ) or mutant (SFTPC ${ }^{173 T / \mathrm{tdT}}$ ) iAEC2s were transduced with an LC3:GFP encoding lentivirus (Twig et al., 2008) at an MOI of $20 \mathrm{in} \mathrm{CK+DCl} \mathrm{media} \mathrm{containing} \mathrm{polybrene} \mathrm{(5} \mathrm{mg/ml).} \mathrm{iAEC2s} \mathrm{were} \mathrm{then} \mathrm{replated} \mathrm{in} \mathrm{growth}$ factor reduced 3D Matrigel (Corning) at a concentration of approximately $400 \mathrm{cells} / \mu \mathrm{l}$ in $\mathrm{CK}+\mathrm{DCl}$ media for expansion. Single cell suspension of transduced iAEC2s was achieved by incubation with $2 \mathrm{mg} / \mathrm{ml}$ dispase (Thermo Fisher Scientific) for 30-60 minutes at $37^{\circ} \mathrm{C}$ and subsequent incubation with $0.05 \%$ trypsin for $12-15$ minutes at $37^{\circ} \mathrm{C}$, as previously described (Jacob et al., 2019 ). Transduced iAEC2s were plated onto Greiner CELLview glass-bottom 4-compartment dishes (Greiner Bio-One GmbH) pre-coated with growth factor reduced Matrigel (Corning) in $\mathrm{CK}+\mathrm{DCl}$ media. After 3 days, iAEC2s were imaged at $37^{\circ} \mathrm{C}$ and $5 \% \mathrm{CO}_{2}$, using a Zeiss LSM 710-Live Duo Scan confocal microscope (Carl Zeiss AG, Oberkochen) (488 nm excitation), and GFP+ punctate autophagosomes were quantified by visual inspection in $40-50$ cells per group before and after 1 hour of incubation with $50 \mathrm{nM}$ bafilomycin A1 (LC Laboratories). LC3 and p62 were further quantified by western blot as described below.

\section{Western Blot Analysis}

Alveolospheres were harvested by incubating with $2 \mathrm{mg} / \mathrm{ml}$ dispase (Thermo Fisher Scientific) for $30-60$ minutes at $37^{\circ} \mathrm{C}$, treated with lysis buffer (RIPA buffer and 1x Roche Complete Protease Inhibitor cocktail), and incubated on ice for 30 minutes. Cellular debris was cleared by centrifugation at $15,000 \mathrm{~g}$ for 20 minutes and supernatants were harvested. Protein concentration was measured using Bio-Rad DC Protein Assay. 5-30 $\mu \mathrm{g}$ of lysates were resolved on pre-cast 12\% Bis-Tris NuPAGE gels (Invitrogen), transferred to PVDF membranes (Bio-Rad), and blotted with primary antibodies (see below and Key resources table) followed by species-specific secondary antibodies conjugated to IR dyes of either 680 or $800 \mathrm{~nm}$ wavelengths (Rockland) at a dilution of 1:10000, as previously described (Hawkins et al., 2015; Maguire et al., 2012). Primary antibodies (see Key resources table) include: surfactant protein B (PT3, a rabbit polyclonal antibody against bovine mature SFTPB; [Beers et al., 1992]; 1:5000), pro-SFTPC (NPRO-SFTPC, a rabbit polyclonal antibody against the Met [10]-Glu [23] domain of rat proSFTPC; [Beers et al., 1994]; 1:3000), mature SFTPC (1:2500, Seven Hills), LC3B (1:750-1:1000, Cell Signaling), SQSTM1/p62 (1:1000, Abcam), $\beta$-actin (1:10000, Sigma), and GAPDH (1:5000, Chemicon). Visualization was accomplished using the Odyssey Imaging System (LiCOR Biosciences).

\section{Respirometry assays}

Alveolospheres were harvested by incubating with $2 \mathrm{mg} / \mathrm{ml}$ dispase (Thermo Fisher Scientific) for $30-60$ minutes at $37^{\circ} \mathrm{C}$, washed, and resuspended in 150-300 $\mu$ l of Seahorse XF Base Media Minimal DMEM (Agilent Technologies) containing $2.8 \mathrm{mM}$ glucose and $0.1 \%$ FBS (pH 7.4). Organoids were seeded in an XF96e Seahorse plate as described (Assali et al., 2019; Taddeo et al., 2020). Briefly, growth factor reduced Matrigel (1.5 $\mu \mathrm{l} /$ well; Corning) was first pipetted in the center measurement zone of each well. The organoid 
suspension (5-10 $\mu \mathrm{l})$ was deposited in the Matrigel-coated measurement zone using a pipette. The plate was then incubated in a non$-\mathrm{CO}_{2}$ incubator for 3.5 minutes to let the Matrigel solidify. Next, $150 \mu \mathrm{l}$ of pre-warmed Seahorse media was slowly added to each well to avoid dislodging the organoids from the Matrigel. The plate containing the organoids was centrifuged at $500 \mathrm{~g}$ for $5 \mathrm{minutes}$ with no brake and subsequently incubated in a non- $\mathrm{CO}_{2}$ incubator for 30-45 minutes prior to running the assay. Oxygen consumption was determined as described (Assali et al., 2019; Taddeo et al., 2020). Port injections were as follows: port A, oligomycin (final concentration $4.5 \mu \mathrm{M} / \mathrm{I}$ ); port B, FCCP diluted in a mixture of $80 \%$ sodium pyruvate and $20 \%$ of $1: 1$ l-leucine/l-glutamine (final concentration $1 \mu \mathrm{M} / \mathrm{I} \mathrm{FCCP} \mathrm{in} 11.4 \mathrm{mM} / \mathrm{I}$ sodium pyruvate and $2.9 \mathrm{mM} / \mathrm{l}$ each of leucine/glutamine); and port C, antimycin A (final concentration $2.5 \mu \mathrm{M} / \mathrm{l})$. Once the Seahorse assay was complete, the plate was kept for mitochondrial content determination. Protein concentration was determined by BCA assay (Thermo Fisher Scientific).

\section{Mitochondrial content determination}

Mitochondrial content was assessed using MitoTracker Deep Red (MTDR) as described (Acin-Perez et al., 2020). Briefly, samples $(5 \mu \mathrm{g})$ were incubated in the presence of $500 \mathrm{nM}$ MTDR for 10 minutes in PBS at $37^{\circ} \mathrm{C}$. The plate was centrifuged at $2000 \mathrm{~g}$ for 10 minutes and media containing MTDR was discarded using a pipette. Then, $100 \mu$ l of PBS was added per well. MTDR fluorescence was measured in a fluorescence plate reader (Tecan, Männedorf, Switzerland) with excitation of $625 \mathrm{~nm}$ and emission of $670 \mathrm{~nm}$. Mitochondrial content was referred as MTDR RFU/ $\mu$ g protein.

\section{Quantification of NF- $\kappa B$ signaling}

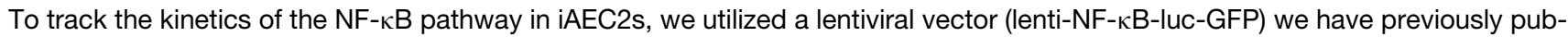
lished (Wilson et al., 2013) that allows independent simultaneous tracking of transduced $\left(\right.$ GFP $^{+}$) cells by flow cytometry and assessment of NF- $\kappa B$ activation levels by luciferase expression. This dual transgenesis vector contains four tandem copies of the canonical NF- $\kappa B$ p50/p65 heterodimer consensus binding sequence located upstream of the minimal TA promoter (TAp), the TATA box of the herpes simplex virus thymidine kinase (HSV-TK) promoter. Located downstream of TAp is the firefly luciferase reporter coding sequence, followed by a constitutively active mammalian ubiquitin C (UBC) promoter driving GFP expression (Figure 7B). Corrected $\left(\right.$ SFTPC $^{\text {tdT/WT}}$ ) or mutant (SFTPC ${ }^{173 T / t d T}$ ) iAEC2s were transduced with the lentiviral vector at an MOI of 20 in CK+DCI media containing polybrene $(5 \mathrm{mg} / \mathrm{ml})$. iAEC2s were then replated in growth factor reduced 3D Matrigel (Corning) at a concentration of approximately $400 \mathrm{cells} / \mu \mathrm{l}$ in CK+DCI media. Thirteen days later, corrected and mutant iAEC2s ( $n=3$ independent droplets) were dissociated into single cells and sorted for transduced $\left(\mathrm{GFP}^{+}\right) \mathrm{SFTPC}^{\text {tdTomato+ }}$ cells which were subsequently resuspended in $75 \mu \mathrm{FACS}$ buffer and plated in a 96-well flat bottom black polystyrene microplate (Corning). Cells were then processed according to the Dual-Glo Luciferase Assay System Protocol (Promega). Firefly luminescence was measured using the Tecan Infinite M200 Pro Micro Plate Reader (Tecan, Männedorf, Switzerland). NF-кB target protein levels were further quantified by Luminex analysis as described below.

\section{Luminex analysis}

Supernatants of corrected and mutant iAEC2s ( $n=3$ independent wells) were filtered through $0.22 \mu \mathrm{m}$ filters (Millipore SCGP00525) and analyzed using a customized Magnetic Luminex ${ }^{\circledR}$ Performance Assay Human HighSensitivity Cytokine Base Kit (R\&D Systems, Inc). Mean fluorescence intensity was measured to calculate final concentration in pg/ml using Bioplex200 and Bioplex Manager 5 software (Bio-Rad) and adjusted to cell number.

\section{Reverse Transcription Quantitative Polymerase Chain Reaction (RT-qPCR)}

RNA was extracted by first lysing cells in Qiazol (QIAGEN) and subsequently using RNAeasy mini kit (QIAGEN) according to the manufacturer's protocol. Complementary DNA (cDNA) was generated by reverse transcription of 150 ng RNA from each sample using MultiScribe Reverse Transcriptase (Applied Biosystems). RT-qPCR was performed using TaqMan Fast Universal PCR Master Mix (Thermo Fisher Scientific) and TaqMan (Applied Biosystems) reagents. The cDNA was diluted 1:4 and $2 \mu \mathrm{L}$ of cDNA were added to each $20 \mu \mathrm{L}$ (for Applied Biosystems StepOne 96-well System) or $12 \mu \mathrm{L}$ (for Applied Biosystems QuantStudio7 384-well system) RTqPCR reaction. Each sample was run in technical triplicates for 40 cycles of PCR and cycle threshold (Ct) values were averaged between triplicates for analysis. Relative gene expression, normalized to $18 \mathrm{~S}$ control, was calculated as fold change in 18S-normalized gene expression, compared to baseline, using the $2^{(-\Delta \Delta C t)}$ method. Baseline expression, defined as fold change $=1$, was set to undifferentiated iPSC levels, or if undetected, a cycle number of 40 was assigned to allow fold change calculations. Adult human lung control RNA was extracted from a healthy donor's distal lung explant. Specifics of primers used are detailed in Key resources table and Table S3.

\section{Isolation of Human Fetal Lung Epithelium}

Week 21 human lung tissues were obtained in the Guttentag laboratory under protocols originally reviewed by the Institutional Review Board at the Children's Hospital of Philadelphia and subsequently reviewed by Vanderbilt University. The cell stocks used in the present studies were donated to the Kotton laboratory for the purpose of providing reference data for this project and their characterization was previously published (Hawkins et al., 2017). "Uncultured naïve lung epithelial cells" were isolated by the overnight culture of lung explants in Waymouth media; a technique that generally yields $86 \pm 2 \%$ epithelial cells with the remaining cells consisting of fibroblasts with $<1 \%$ endothelial cells (Wade et al., 2006). 
RNA sequencing and bioinformatic analyses

For bulk RNA sequencing (RNA-seq) comparing corrected (SFTPC ${ }^{\text {tdT/WT }}$ ) and mutant (SFTPC ${ }^{173 T / t d T}$ ) iAEC2s at two different time points (day 114 and day 131), experimental triplicate $(n=3)$ samples of purified RNA extracts were harvested from each group of samples as follows. Alveolospheres were harvested from 3D Matrigel by incubating with $2 \mathrm{mg} / \mathrm{ml}$ dispase (Thermo Fisher Scientific) for 30-60 minutes at $37^{\circ} \mathrm{C}$, washed twice with PBS, and RNA was extracted using the Qiazol and miRNeasy mini kit (QIAGEN) according to manufacturer's instructions. Flow cytometry analysis confirmed that these samples consisted of $\geq 95 \%$ SFTPC ${ }^{\text {tdTomato+ }}$ cells. mRNA was isolated from each sample using magnetic bead-based poly(A) selection, followed by synthesis of cDNA fragments. The products were end-paired and PCR-amplified to create each final cDNA library. Sequencing of pooled libraries was done using a NextSeq 500 (Illumina). The quality of the raw sequencing data was assessed using FastQC v.0.11.7. Sequence reads were aligned to a combination of the human genome reference (GRCh38) and the tdTomato reporter sequence, using STAR v.2.5.2b (Dobin et al., 2013). Counts per gene were summarized using the featureCounts function from the subread package v.1.6.2. The edgeR package v.3.25.10 was used to import, organize, filter and normalize the data. Genes that were not expressed in at least one of the experimental groups were filtered out (keeping only genes that had at least 10 reads in at least 3 libraries, that is, a worthwhile number of samples as determined by the replicate number in the design matrix. The TMM method was used for normalization. Principal Component Analysis (PCA) and Multidimensional Scaling (MDS) were used for exploratory analysis, to assess sample similarities and potential batch effects. Subsequently, the limma package v3.39.19 (Law et al., 2016) with its voom method, namely, linear modeling and empirical Bayes moderation was used to test differential expression (moderate $t$ tests). $P$ values were adjusted for multiple testing using Benjamini-Hochberg correction (false discovery rate-adjusted $p$ value; FDR). Differentially expressed genes between the groups in each experiment were visualized using Glimma v 1.11.1, and FDR < 0.05 was set as the threshold for determining significant differential gene expression. Gene set analysis was performed with Hallmark, KEGG, and GO Biological Process gene sets using the Camera package (Wu and Smyth, 2012).

For single-cell RNA sequencing (scRNA-seq) comparing corrected (SFTPC ${ }^{\text {tdT/WT}) \text { ) and mutant (SFTPC }}{ }^{173 T / t_{d T}}$ ) iAEC2s at two different time points (day 30 and day 113), cells were dissociated for sorting as described previously. Cells were sorted as described previously for viable (calcein blue+) SFTPC ${ }^{\text {tdTomato+ }}$ cells and resuspended in FACS buffer at a concentration of $800-1000$ cells/ $\mu$ l. For the scRNA-seq experiment comparing corrected and mutant iAEC2s on day 114 (Figure S3), all live cells (calcein blue+) irrespective of SFTPC ${ }^{\text {tdTomato }}$ expression were included. Single cells were captured for sequencing library preparation using a 10X Chromium (10X Genomics, Pleasanton, CA) instrument. scRNA-seq libraries were prepared according to the Single Cell $3^{\prime}$ v3 Reagent Kits User Guide (10X Genomics). Cellular suspensions were loaded on a Chromium Controller instrument (10X Genomics) to generate single-cell Gel Bead-In-Emulsions (GEMs). Reverse transcription (GEM-RT) was performed in a Veriti 96-well thermal cycler (Thermo Fisher Scientific). After RT, GEMs were harvested and the cDNAs were amplified and cleaned with SPRIselect Reagent Kit (Beckman Coulter). Indexed sequencing libraries were constructed using the Chromium Single-Cell 3' Library Kit (10X Genomics) for enzymatic fragmentation, end-repair, A-tailing, adaptor ligation, ligation cleanup, sample index PCR, and PCR cleanup. The barcoded sequencing libraries were quantified by quantitative PCR using the KAPA Library Quantification Kit (KAPA Biosystems, Wilmington, MA). Sequencing libraries were loaded on a NextSeq500 (Illumina) with a custom sequencing setting (26bp for Read 1 and 98bp for Read 2), to obtain a sequencing depth of $\sim 50 \mathrm{~K}$ reads/cell. Fastq files were generated using bcl2fastq v.2.2 and Cellranger v.3.0.2. The sequence files were mapped to the human genome reference (GRCh37) including the TdTomato reporter. We used Seurat v.3 to further process the data. We estimated the doublet rate according to the 10X Chromium guidelines, in proportion to the density of cells loaded. These rates were used to flag potential doublets based on their gene and UMI counts. Cells with fewer than 800 genes detected were also filtered out, as well as cells with high percentage of counts mapping to mitochondrial genes (thresholds set by manual inspection for each dataset, between $25 \%$ to $35 \%$ ). For each experiment, the mutated and corrected samples were merged and then normalized using SCTransform, with cell degradation effect regressed out. After an initial linear dimensionality reduction (PCA), we used UMAP projections to represent the data and the Louvain algorithm was used for clustering. Differential expression tests were done with MAST, applying independent prior to gene testing (minimum detection percentage of $10 \%$ in at least one of the populations, minimum average log-fold change of 0.15 between the two populations). Gene signature enrichment scores for iAT2 markers and cell cycle stage were computed using Seurat. The significance of the differences in these scores between corrected and mutant samples was tested using Welch Two Sample t tests. The scRNA-seq data discussed in this publication have been deposited in NCBI's Gene Expression Omnibus (GEO; accession number GSE160801).

Proteomic and phosphoproteomic analysis

Cell lysis and trypsin digestion

For proteomic/phosphoproteomic analyses experimental triplicate $(n=3)$ samples were dissociated for sorting as described previously. Cells were sorted as described previously for viable (calcein blue+) SFTPC ${ }^{\text {tdTomato+ }}$ cells, washed with PBS, pelleted, and stored at $-80^{\circ} \mathrm{C}$. Cell pellets were thawed and individually placed in $300 \mu$ of lysis buffer composed of $8 \mathrm{M}$ guanidinium chloride (GuHCl), $50 \mathrm{mM}$ ammonium bicarbonate, $5 \mathrm{mM}$ dithiothreitol (DTT), phosphatase (PhosSTOP, Roche) and protease (Complete, Mini, EDTA-free Protease Inhibitor Cocktail, Roche) inhibitors and then sonicated on ice using a Branson probe (40 s, in $2 \mathrm{~s}$ pulses). Lysates were alkylated in $12 \mathrm{mM}$ iodoacetamide. Protein concentration was quantified by the Bradford protein assay (Bio-Rad) and samples were diluted with 7 volumes of $50 \mathrm{mM}$ ammonium bicarbonate to bring the urea concentration to $1 \mathrm{M}$. Each sample was then digested overnight at $37^{\circ} \mathrm{C}$ with $2 \mu \mathrm{g}$ sequencing-grade trypsin (Pierce Trypsin Protease, MS Grade, Thermo Fisher Scientific). 
Tandem Mass Tag (TMT) labeling and reserve phase high performance liquid chromatography (LC)

After digestion, the reactions were terminated by adding trifluoroacetic acid (TFA) to $0.1 \%$, de-salted (Waters Sep-Pak), and resuspended in $100 \mathrm{mM}$ HEPES buffer. Total peptide concentration was quantified using the Pierce Quantitative Colorimetric Peptide Assay (Pierce Quantitative Colorimetric Peptide Assay, Thermo Fisher Scientific). Then $100 \mu \mathrm{g}$ peptide from each sample (volume adjusted to $100 \mu \mathrm{l}$ with $100 \mathrm{mM}$ HEPES buffer) was mixed with a unique amine-reactive heavy isotope-coded isobaric TMT (TMT11 plex) reagent, allowing for multiplex of samples prior to LC/MS. After pooling, the samples were desalted and dried, and a total of about $0.6 \mathrm{mg}$ of labeled peptide was suspended in $300 \mu \mathrm{l}$ buffer containing $0.1 \%$ ammonium hydroxide and $2 \%$ acetonitrile. This mixture was injected onto a reverse-phase Waters Xbridge C18 HPLC column (Waters) connected to an Agilent 1100 series HPLC system. Peptides were eluted from the column using a gradient of mobile phase $\mathrm{A}\left(2 \% \mathrm{ACN}, 0.1 \% \mathrm{NH} \mathrm{HH}_{4} \mathrm{OH}\right.$ to mobile phase $\mathrm{B}\left(98 \% \mathrm{ACN}, 0.1 \% \mathrm{NH}_{4} \mathrm{OH}\right)$ over 48 minutes. Fractions were collected every minute, and every $12^{\text {th }}$ fraction was pooled, yielding 12 final fractions, each containing approximately $100 \mu \mathrm{g}$ peptide. Most of each fraction (95\%) was set aside for phospho-peptide enrichment using Fe-NTA magnetic beads (Cube Biotech) while the remaining 5\% was analyzed separately by mass spectrometry as bulk proteome measurements, totaling 24 injections.

\section{Mass Spectrometry (MS)}

Fractionated peptide samples were analyzed using a Q Exactive HFX mass spectrometer connected to Easy nLC 1200 ultra high-pressure chromatography system (Thermo Scientific). Lyophilized peptides were resuspended in mobile phase A solvent ( $0.1 \%$ formic acid and $2 \%$ acetonitrile). The samples were loaded onto a reverse-phase nano-trap column with mobile phase A, (75 $\mu \mathrm{m}$ i.d. $\times 2 \mathrm{~cm}$, Acclaim PepMap100 C18 $3 \mu \mathrm{m}, 100 \AA$, Thermo Scientific) and were separated over an EASY-Spray column, (ES803A, Thermo Scientific) using a gradient ( $2 \%$ to $35 \%$ over $120 \mathrm{~min}$ ) of mobile phase B (0.1\% formic acid, $80 \%$ acetonitrile) at a flow rate of $250 \mathrm{nl} / \mathrm{min}$. The mass spectrometer was operated in positive ion mode with a capillary temperature of $275^{\circ} \mathrm{C}$, and with a potential of $2100 \mathrm{~V}$ applied to the emitter. All data were acquired with the mass spectrometer operating in automatic data dependent switching mode. A high resolution $(60,000)$ MS precursor ion scan $(350-1500 \mathrm{~m} / \mathrm{z}$ range) was performed to select the 12 most intense ions for subsequent fragmentation and MS/MS analysis using HCD (NCE $33 @ 45,000$ resolution).

Peptide identification

Resulting RAW files were converted and searched using the MaxQuant platform (version 1.6.7.0; https://maxquant.org/) under standard settings against the Uniprot human reference protein sequence database (downloaded: April 16, 2019) allowing for two missed trypsin cleavage sites, variable modifications: phosphorylation at $\mathrm{S}, \mathrm{T}$, and $\mathrm{Y}$ residues, $\mathrm{N}$-terminal acetylation, and methionine oxidation. Carbamidomethylation of cysteine residues was set as a fixed modification. Peptide precursor ions were searched with a maximum mass deviation of $4.5 \mathrm{ppm}$ and fragment ions with a maximum mass deviation of 20 ppm. Peptide and protein identifications were filtered at 1\% FDR using the target-decoy database search strategy. The MaxQuant output files designated "Phospho(STY)sites" and "ProteinGroups" were used for data normalization and other statistical analysis.

Data analysis and pathway enrichment

Bioinformatic analysis was performed using R: A language and environment for Statistical Computing (R Foundation for Statistical Computing, Vienna, Austria. http://www.R-project.org), version 3.6.1. The protein groups and phosphosite tables from MaxQuant were filtered to eliminate entries labeled as reverse hits, potential contaminants, and "only identified by site." Protein quantitation required at values in at least $70 \%$ of TMT channels. The TMT-reporter intensity values were log2 transformed and Loess-normalized. For differential analysis, the LIMMA (Ritchie et al., 2015) R package was used to fit a linear model accounting for the cell lines. Moderated $t$ tests were corrected with the Benjamini-Hochberg method for FDR. Gene set enrichment analysis was performed using the fgsea R package (Korotkevich et al., 2019) using the Hallmark (H) and Curated (C2) gene set libraries from MSigDB (Subramanian et al., 2005) with ranked lists where the gene rank is defined as - $\log (p$ value)*sign(log2 fold change) (Reimand et al., 2019). In the case of duplicate proteins mapping to a common gene symbol, the one with the highest absolute value rank was retained. Heatmaps were generated with the ComplexHeatmap R package (Gu et al., 2016). The mass spectrometry proteomics data have been deposited to the ProteomeXchange Consortium via the Proteomics Identifications (PRIDE) partner repository (Deutsch et al., 2020; PerezRiverol et al., 2019) with the dataset identifier PRIDE: PXD022334.

\section{QUANTIFICATION AND STATISTICAL ANALYSIS}

Statistical methods relevant to each figure are outlined in the figure legend. In brief, unpaired, two-tailed Student's t tests were used to compare quantitative analyses comprising two groups of $n=3$ or more samples, or one-way ANOVAs with multiple comparisons were used to compare three or more groups. Further specifics about the replicates used in each experiment are available in the figure legends. In these cases, a Gaussian distribution and equal variance between samples was assumed as the experiments represent random samples of the measured variable. The $p$ value threshold to determine significance was set at $p=0.05$. $p$ value annotations on graphs are as follows: ${ }^{*} p<0.05,{ }^{\star *} p<0.01,{ }^{* \star *} p<0.001,{ }^{\star * \star *} p<0.0001$. Data for quantitative experiments are typically represented as the mean with error bars representing the standard deviation or standard error of the mean, as specified in the figure legends.

Statistical analysis on imaged alveolospheres was performed using Prism 7 (GraphPad Software, San Diego, CA). Each organoid was treated as an experimental replicate and unpaired Student's t test was used to determine statistically significant differences. 
Cell Reports, Volume 36

Supplemental information

Patient-specific iPSCs carrying an SFTPC mutation

reveal the intrinsic alveolar epithelial dysfunction

at the inception of interstitial lung disease

Konstantinos-Dionysios Alysandratos, Scott J. Russo, Anton Petcherski, Evan P. Taddeo, Rebeca Acín-Pérez, Carlos Villacorta-Martin, J.C. Jean, Surafel Mulugeta, Luis R. Rodriguez, Benjamin C. Blum, Ryan M. Hekman, Olivia T. Hix, Kasey Minakin, Marall Vedaie, Seunghyi Kook, Andrew M. Tilston-Lunel, Xaralabos Varelas, Jennifer A. Wambach, F. Sessions Cole, Aaron Hamvas, Lisa R. Young, Marc Liesa, Andrew Emili, Susan H. Guttentag, Orian S. Shirihai, Michael F. Beers, and Darrell N. Kotton 
Figure S1

A
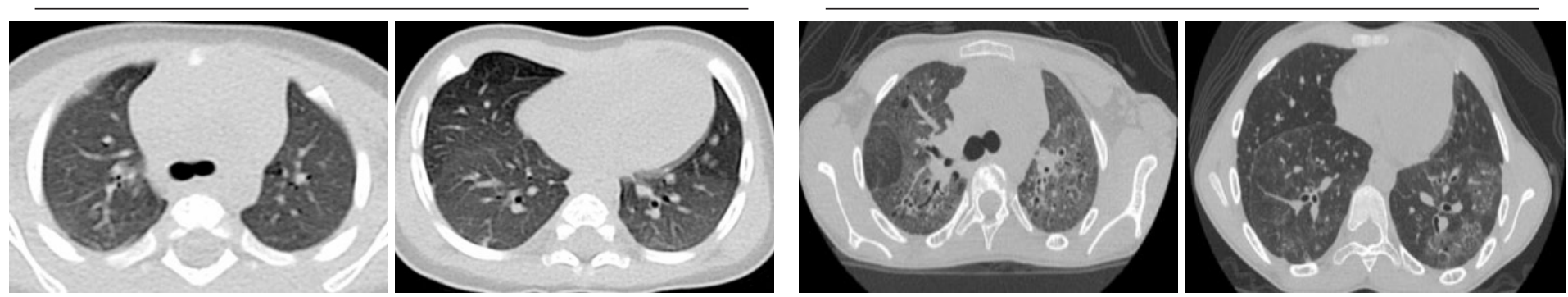

B

SFTPC locus

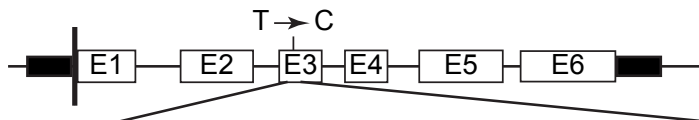

GGTGGCTCCATGCCCTTTCCCCAGGTTCTGGAGATGAGCACTGGGGCGCCGGAAGCCCAGCAACGCCTGGCCCTGAGTGAG

gDNA target sequence $\overline{\text { PAM }}$

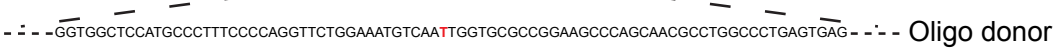

$\rightarrow-E_{1}-E_{2}-\frac{T}{E 3-}-E_{4}-E 5-\left[-E_{6}-\right.$

C

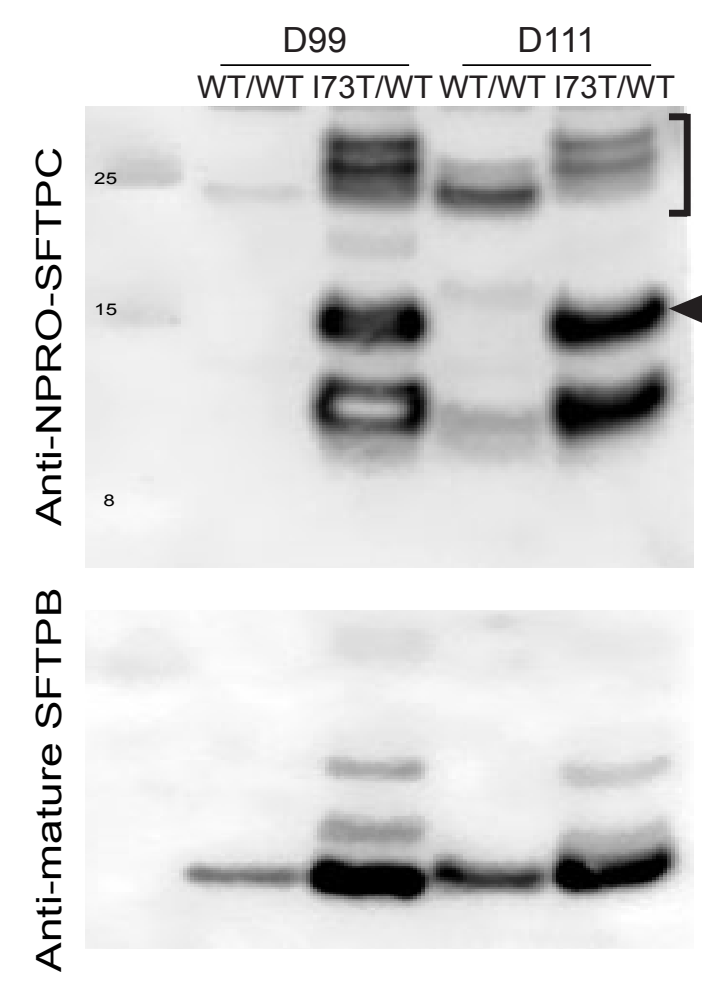

$$
\text { B }
$$

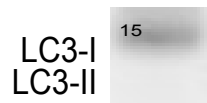

p62

$\beta$ actin

D

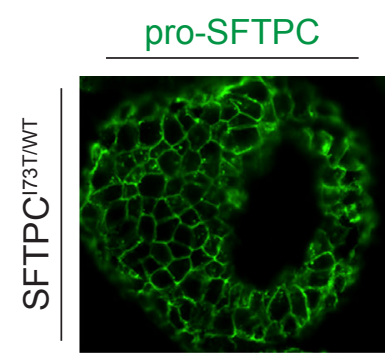

$\beta$ actin
SPC2

$\mathbf{E}$ SPC2Corr

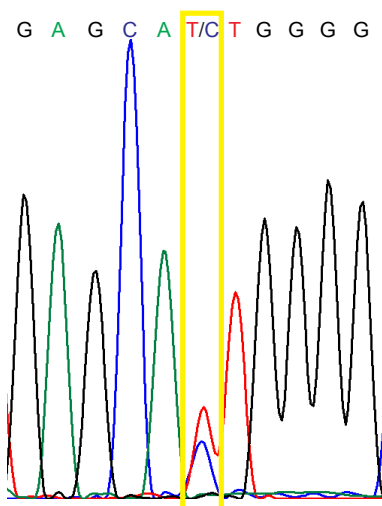

SPC2Corr

G A/T G/C C/A A $\quad T \quad T \quad G \quad G \quad G / T$ G

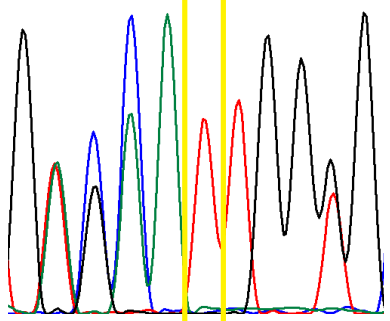

SPC2

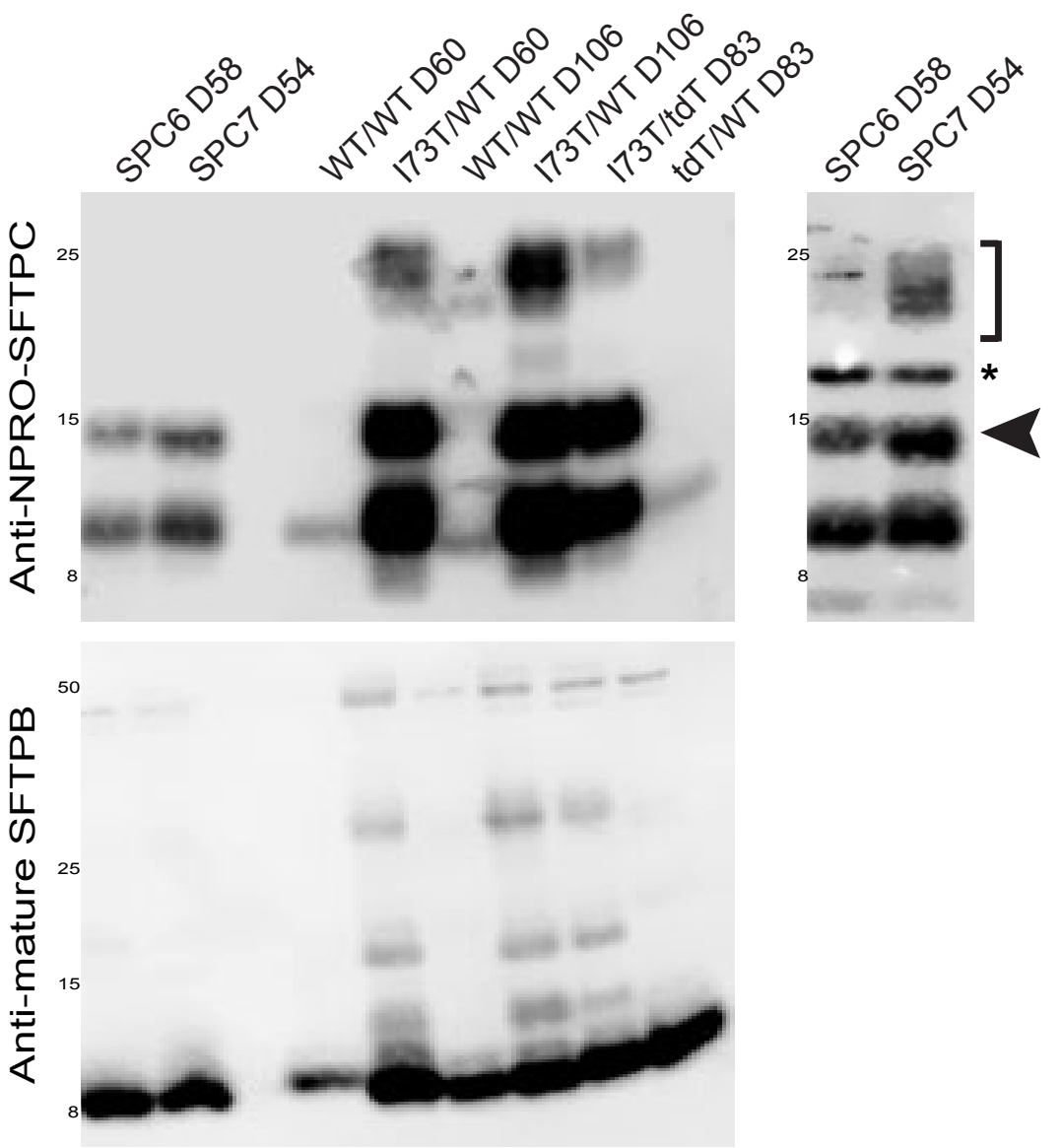


Figure S1. Pro-SFTPC misprocessing and autophagy perturbations in iAEC2s derived from patient-specific iPSCs carrying the $S F T P C^{I 73 T / W T}$ mutation compared to controls generated by CRISPR-mediated gene editing of the $S F T P C$ locus, related

to Figure 3 and Figure 5. (A) Chest CT of the SPC6 donor at 3 months of age reveals bilateral diffuse ground glass opacities; CT chest of the SPC7 donor at age 12 years reveals bilateral ground glass opacities and traction bronchiectasis. (B) Footprint free CRISPR-mediated editing of the SFTPC locus to engineer correction of patient-specific SPC2 iPSCs. The I73T mutation in SFTPC exon 3: genomic sequence with the c.218 T $>$ C (p.I73T) mutation is shown in red, the CRISPR guide RNA target sequence shown in green, the protospacer adjacent motif (PAM) cutting site in blue, and the oligo-based donor design with corrected base shown in red. Pre- and post-correction DNA sequencing chromatograms with yellow boxes show the c.218 mutation site sequence. (C) Representative Western blot of SFTPC ${ }^{\mathrm{WT} / \mathrm{WT}}$ and SFTPC ${ }^{\mathrm{I} 3 \mathrm{~T} / \mathrm{WT}}$ iAEC2 lysates from the iPSC clones described in (B) at the indicated differentiation time points for pro-SFTPC (NPRO-SFTPC), mature SFTPB, p62 and LC3 with $\beta$ actin as loading control. SFTPC ${ }^{173 T /}$ WT iAEC2s accumulate large amounts of the primary translation product (bracket) and misprocessed intermediate forms (arrowhead). In SFTPC ${ }^{\mathrm{WT} / \mathrm{WT}}$ iAEC2s, both the primary translation product and major processing intermediate forms are detected. SFTPC $^{\mathrm{I73T} / \mathrm{WT}}$ iAEC2s accumulate a larger amount of mature $8 \mathrm{kDa}$ SFTPB. SFTPC ${ }^{\text {I73T/WT }}$ iAEC2s demonstrate increased accumulation of both LC3 and p62 compared to SFTPC ${ }^{\mathrm{WT} / \mathrm{WT}}$ iAEC2s, similarly to SFTPC ${ }^{\mathrm{I} 3 \mathrm{~T} / \mathrm{tdT}}$ iAEC2s. See also Figure 5B. (D) Representative immunofluorescence microscopy of SFTPC ${ }^{\mathrm{I73T} / \mathrm{WT}}$ iAEC2s for pro-SFTPC (green), EPCAM (red), and DNA (Hoechst, blue) reveals SFTPC protein localized to SFTPC ${ }^{\mathrm{I} 33 \mathrm{~T} / \mathrm{WT}}$ iAEC2 plasma membranes. Scale bars: $10 \mu \mathrm{m}$. (E) Representative Western blot of protein lysates from differentiated cultures derived from two additional patient-specific (SPC6 and SPC7) iPSC

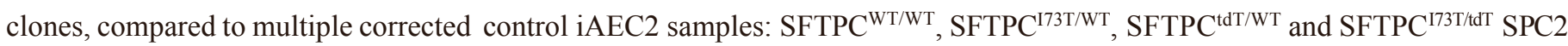
iAEC2 lysates at the indicated time points for pro-SFTPC (NPRO-SFTPC) (left panel) and mature SFTPB (lower panel) with $\beta$ actin as loading control. Both SPC6 and SPC7 iAEC2s produce mature 8 kDa SFTPB and misprocess SFTPC. Longer exposure of the SPC6 and SPC7 lanes (right panel) shows that both SPC6 and SPC7 iAEC2s accumulate a higher molecular weight SFTPC primary translational product (bracket) and misprocessed intermediate forms (arrowhead) similar to SFTPC ${ }^{\mathrm{I73T} / \mathrm{WT}}$ and $\mathrm{SFTPC}^{\mathrm{l73T} / \mathrm{tdT}}$ mutant iAEC2s. Asterisk represents a non-specific band. 


\section{Figure S2}

$A_{\cap}$

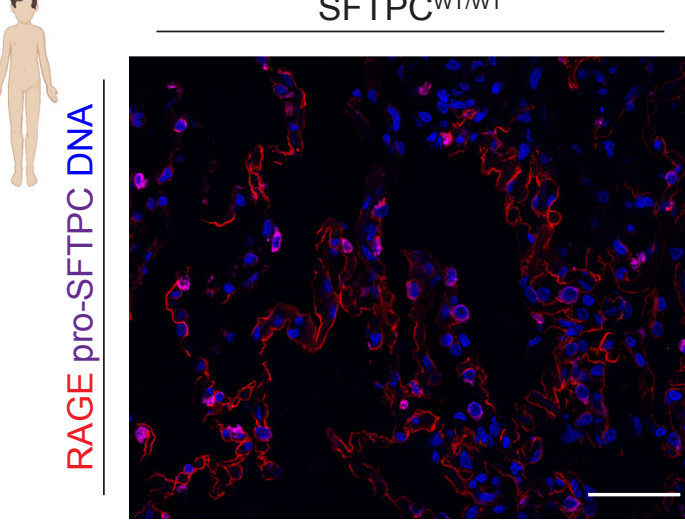

SFTPC 173T/WT

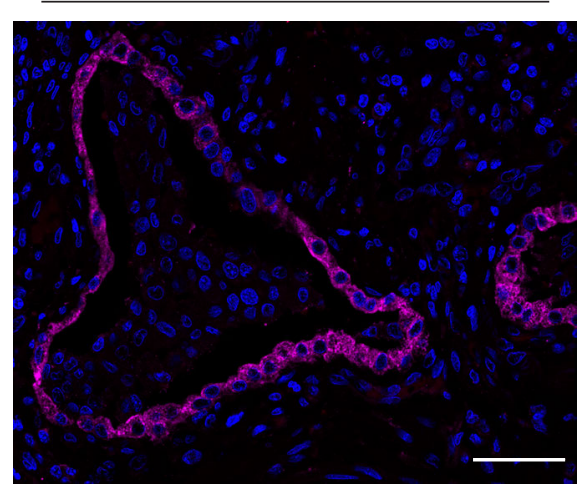

B

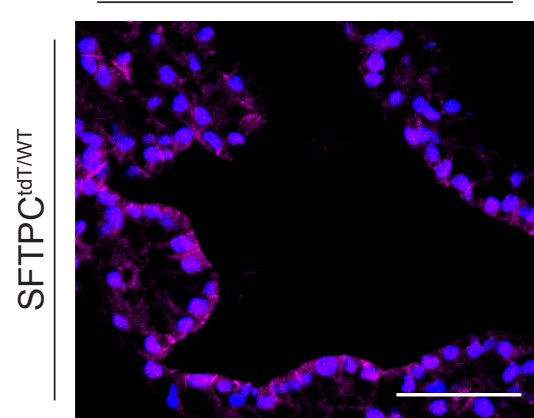

MUC1 DNA
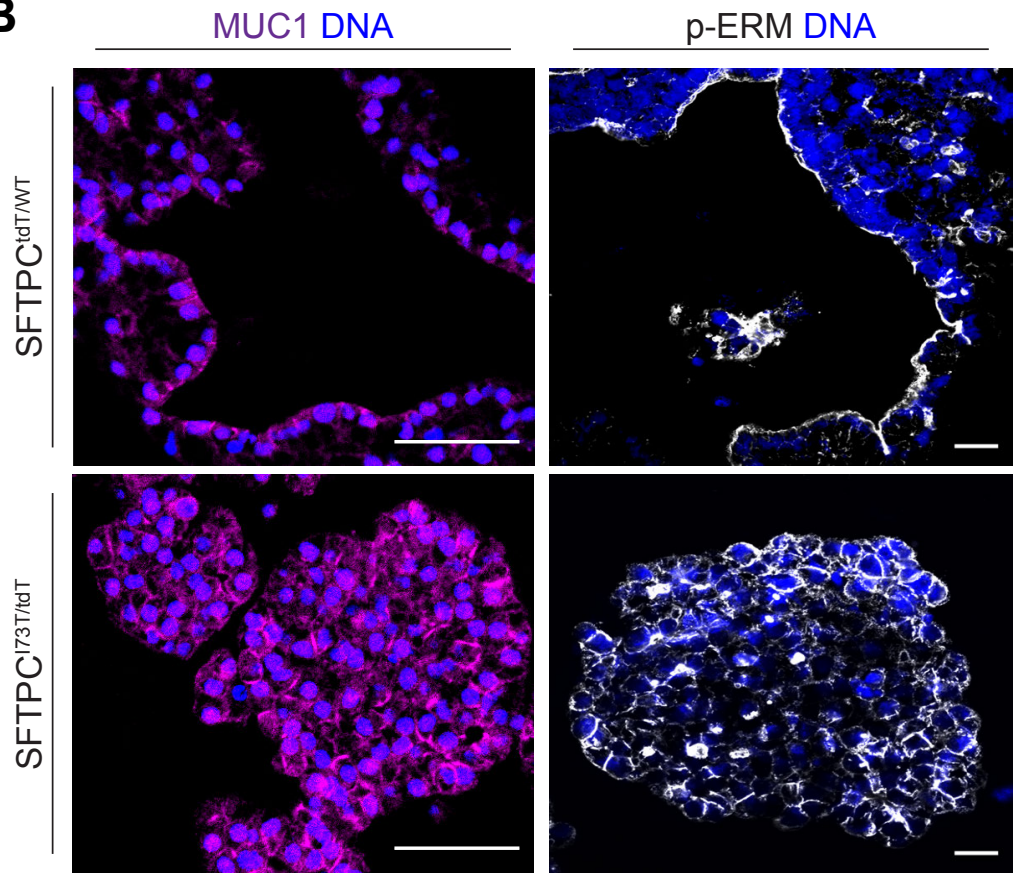

CRB3

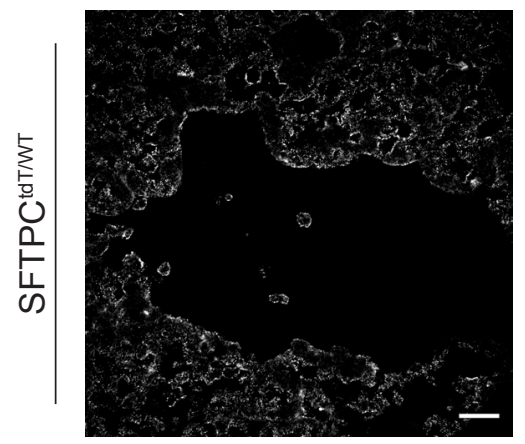

눌
$\mathrm{CDH} 1$
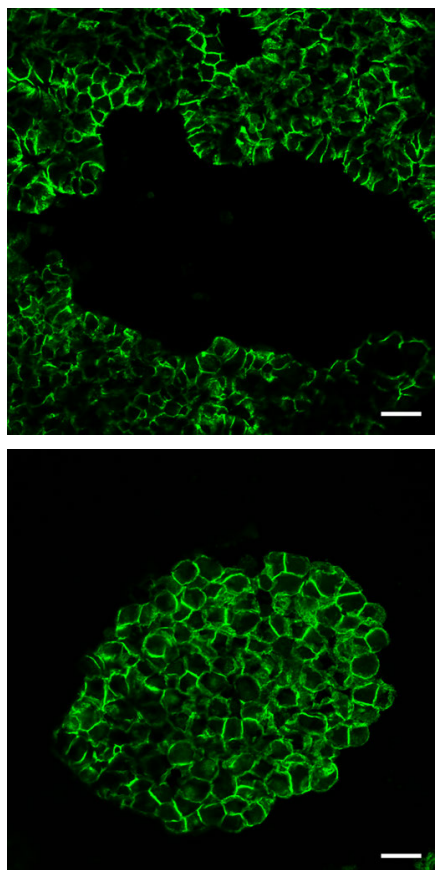

Merge
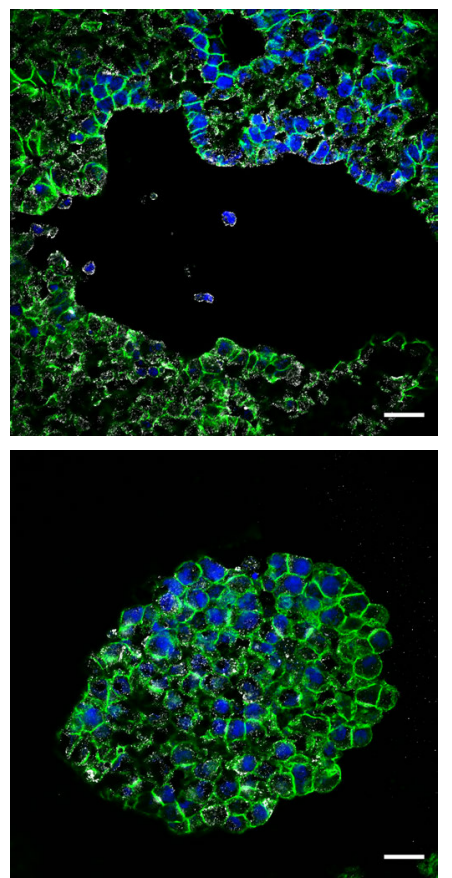
Figure S2. Paucity of AEC1s in human $S F T P C^{173 T}$ lungs and loss of apical-basal polarity in $S F T P C^{173 T / t d T}$ iAEC2s, related to

Figure 3. (A) Representative tile scan confocal immunofluorescence microscopy of distal sections of the SPC2 donor and healthy donor lung explants stained for RAGE (red), pro-SFTPC (magenta), and DNA (Hoechst, blue). Scale bars: $50 \mu \mathrm{m}$. (B)

Representative confocal immunofluorescence microscopy of SFTPC ${ }^{\mathrm{tdT} / \mathrm{WT}}$ and SFTPC ${ }^{\mathrm{I73T} / \mathrm{dT}}$ iAEC2 cultures for MUC1 (magenta), p-ERM (grey), CRB3 (grey), E-Cadherin/CDH1 (green), and DNA (Hoechst, blue) reveals loss of apical-basal polarity in SFTPC $^{173 T / d \mathrm{dT}}$ iAEC2s. Scale bars: $50 \mu \mathrm{m}$. 
Figure S3

A

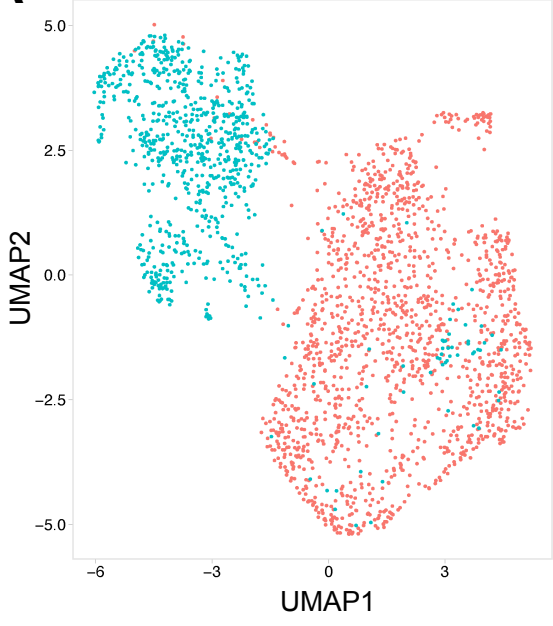

C $\quad S F T P B$

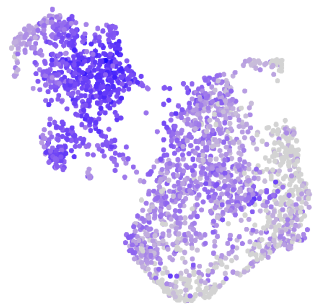

AEC2 8-gene set

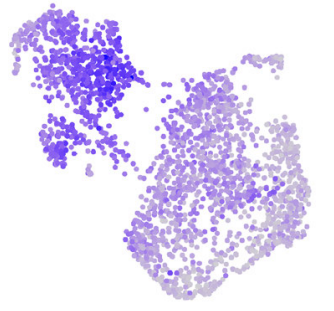

SFTPC

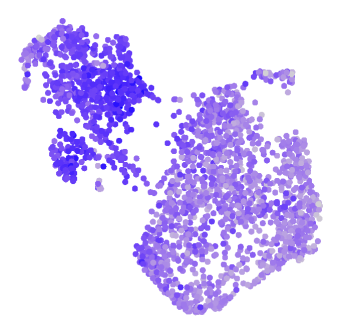

ALB
tdT/WT

B

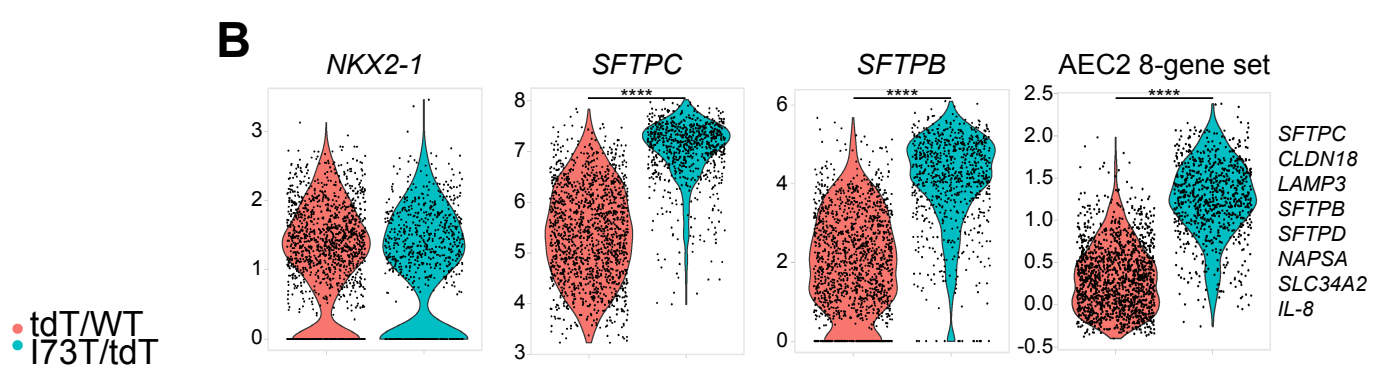

NAPSA

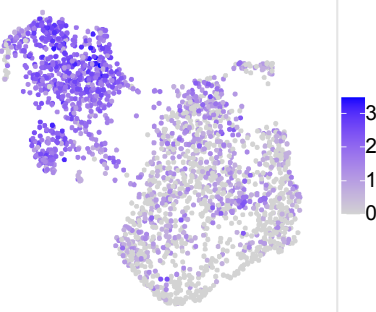

AEC2 5-gene set

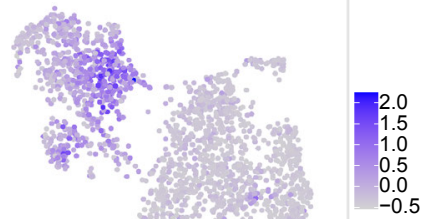

AFP

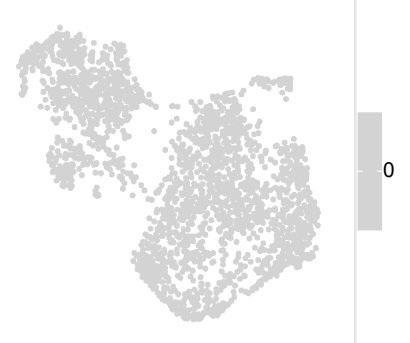

CDX2

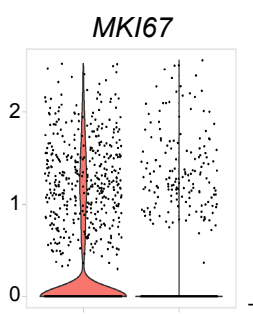

S Phase

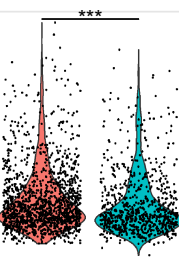

G2M Phase

$-0.2$
D。

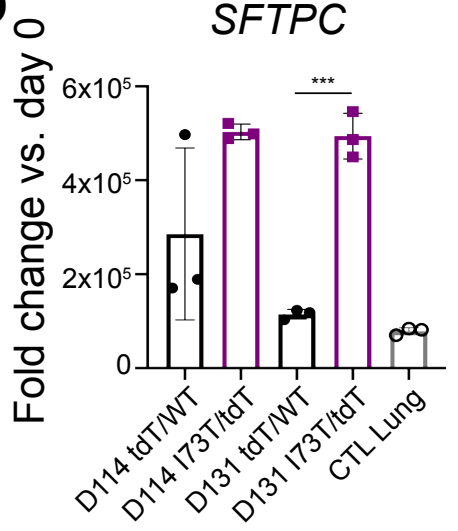

- SLC34A2

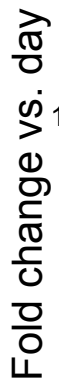

E
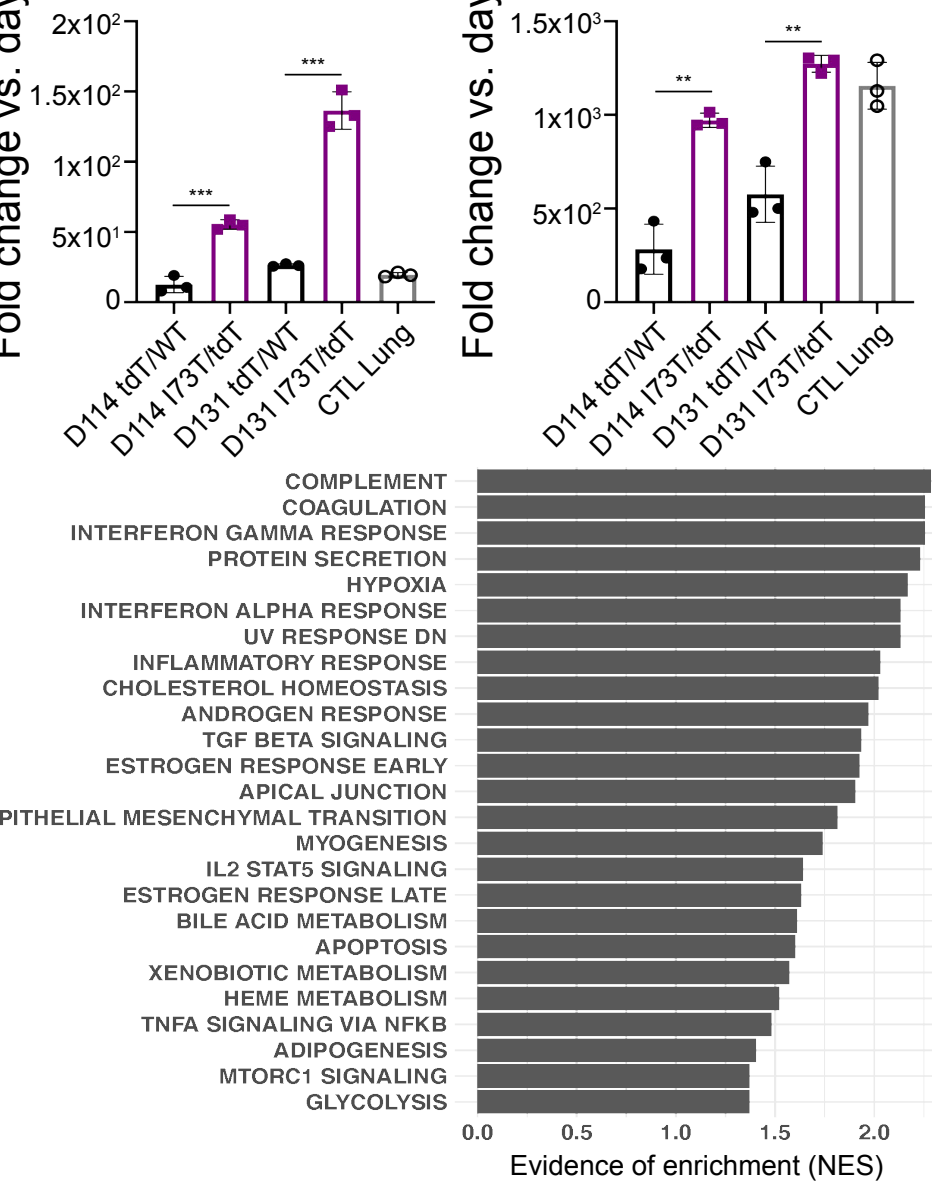
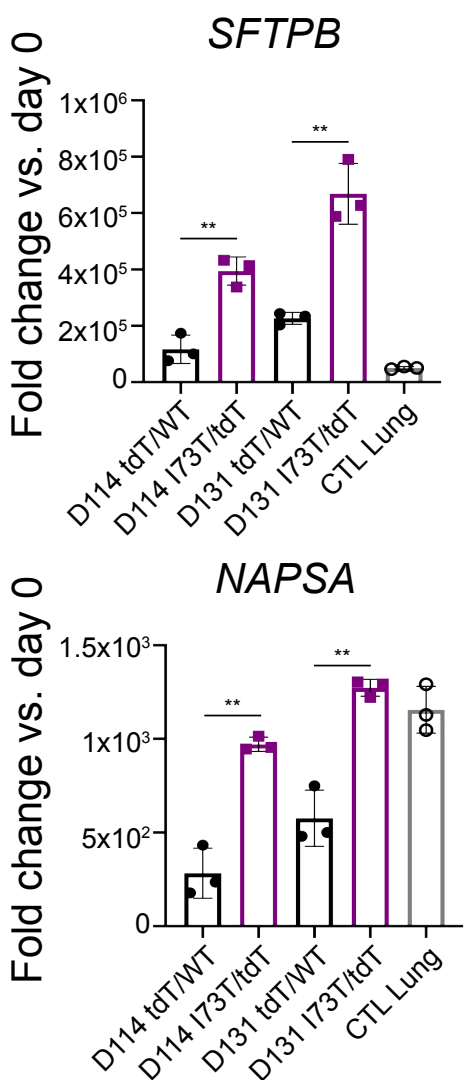

$\pi$

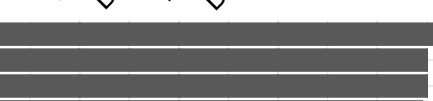


Figure S3. Characterization of gene expression in SFTPC ${ }^{173 T / t d T}$ iAEC2s reveals pathway perturbations and higher

expression of AEC2 marker genes, related to Figure 4. (A) Clustering of day 114 scRNA-seq transcriptomes in each indicated iAEC2 population using Uniform Manifold Approximation Projection (UMAP). All live iAEC2s, irrespective of SFTPC ${ }^{\text {tdTomato }}$ expression were included. (B) Violin plots showing normalized expression for indicated genes or cell cycle phase in day 114 SFTPC $^{\text {tdT/WT }}$ and SFTPC ${ }^{\text {I73T/dT }}$ iAEC2s by scRNA-seq. (C) Normalized gene expression overlayed on UMAP plots for the indicated transcripts or gene sets. (D) RT€PCR showing fold change in gene expression compared to day 0 (2(- $\Delta \Delta \mathrm{Ct})$ in day 114 and day 131 SFTPC $^{\text {tdT/WT }}$ and SFTPC ${ }^{\text {I73T/dtT }}$ iAEC2s as well as control samples of an adult control human distal lung explant (CTL Lung). (mean $\pm \mathrm{SD} ; \mathrm{n}=3$ experimental replicates of independent wells of a differentiation). (E) Gene set enrichment analysis (GSEA, Camera of day 113 scRNA-seq from Figure 4A, using HALLMARK gene sets) of significantly upregulated gene sets $($ FDR $<0.05)$ in SFTPC ${ }^{173 T / t d T}$ compared to SFTPC ${ }^{\text {tdT/WT }}$ iAEC2s. NES: normalized enrichment score. $* * p<0.01, * * * p<0.001$, $* * * * \mathrm{p}<0.0001$ by unpaired, two-tailed Student's $t$-test for all panels. 
Figure S4

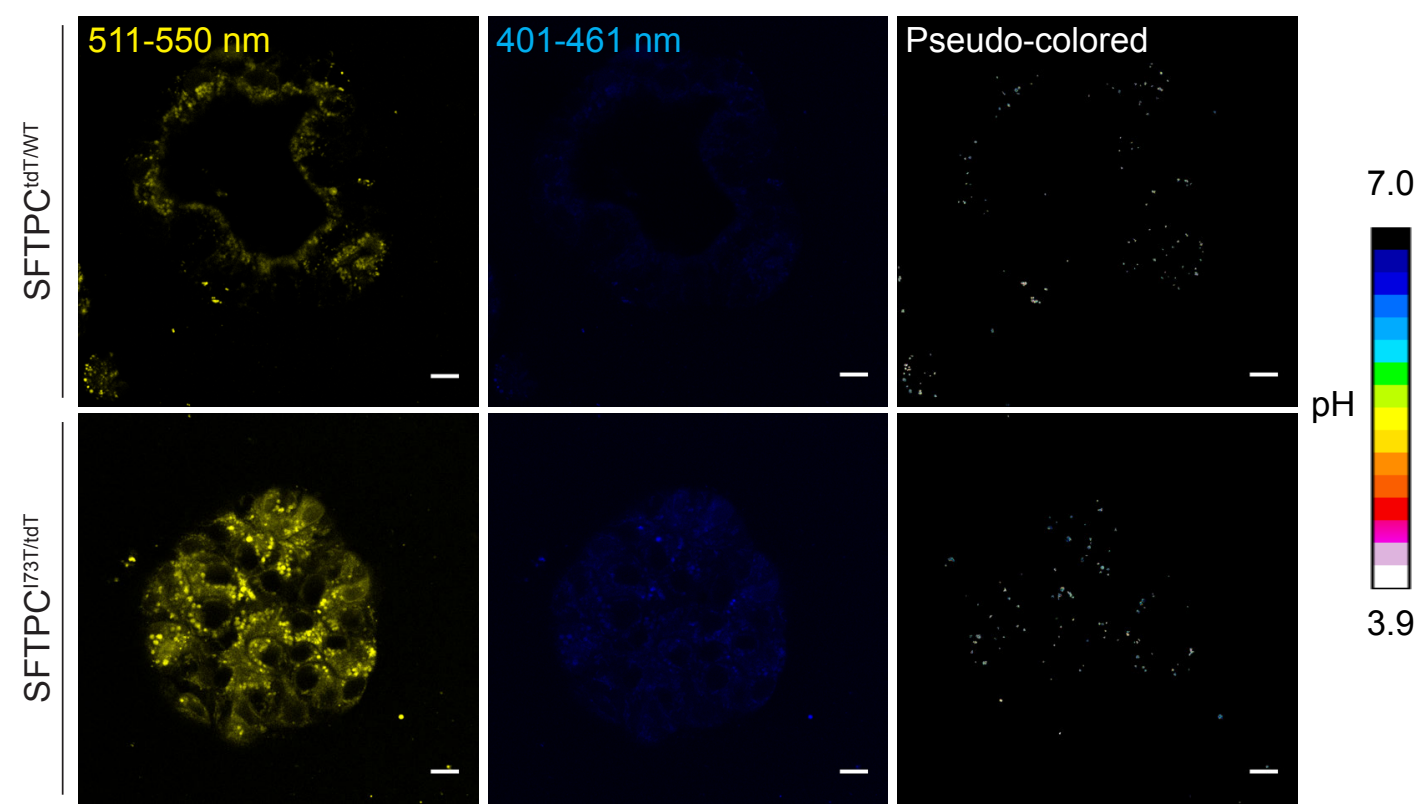

Figure S4. SFTPC ${ }^{173 T / t d T}$ iAEC2 lysosomes demonstrate a lower pH when compared to SFTPC tdT/WT $^{\text {iAEC2 }}$ lysosomes, related to Figure 5K. Representative live-cell confocal fluorescence microscopy of SFTPC ${ }^{\mathrm{tdT} / \mathrm{WT}}$ and SFTPC $^{173 T / d T}$ iAEC2s stained with Lysosensor yellow/blue-dextran and imaged using two filter ranges, 401-461 nm (blue) and 511-550 nm (yellow). Subcellular organelles with lower pH are identified as pixels with increased yellow/ blue ratio. Pseudo-colored images reflect the organelle pH. Scale bars: $10 \mu \mathrm{m}$ (see also Figure 5K). 


\section{Figure S5}

A

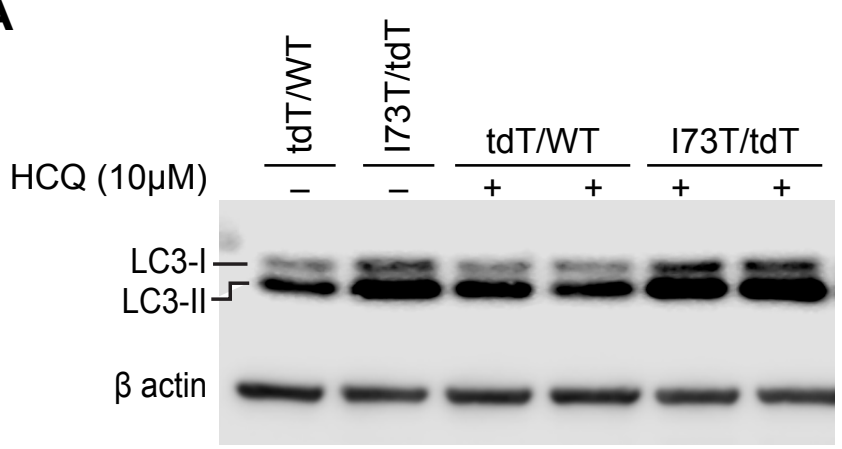

B
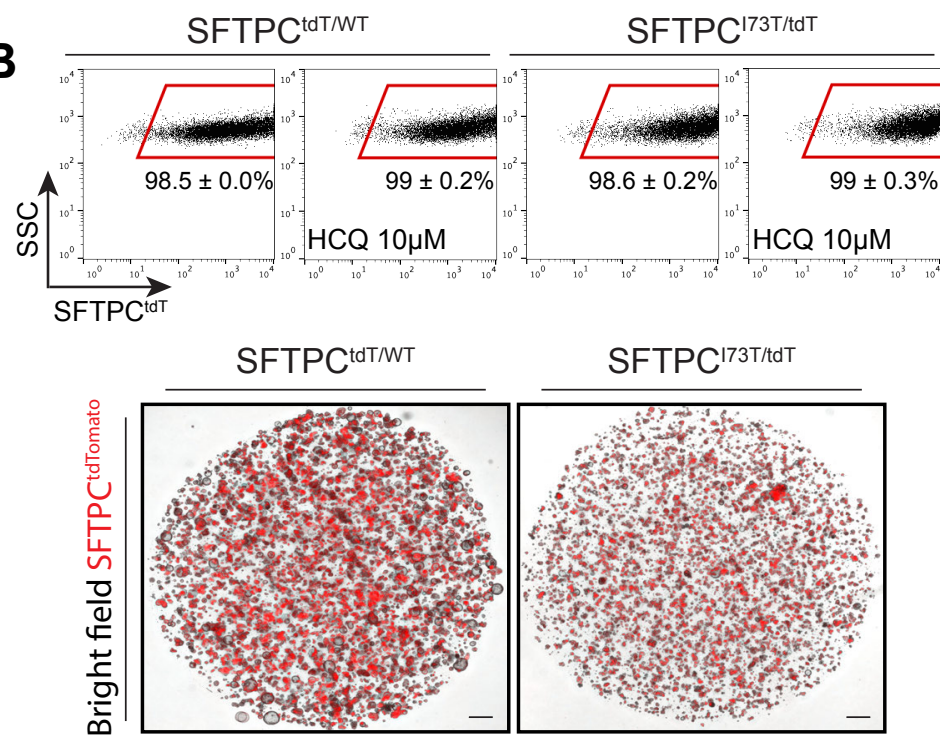

C
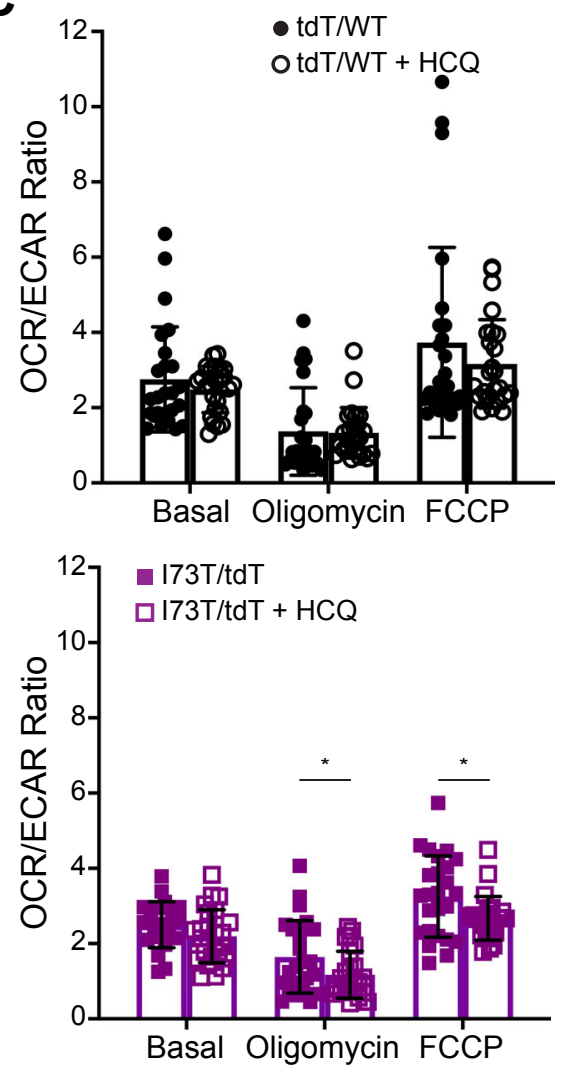

Figure S5. Hydroxychloroquine treatment aggravates the autophagic flux block and metabolic reprogramming in SFTPC $^{173 T}$-expressing iAEC2s, related to Figure 7G, H. (A) SFTPC ${ }^{\mathrm{tdT} / \mathrm{WT}}$ or SFTPC ${ }^{173 T / \mathrm{dT} T}$ iAEC2s were treated with either hydroxychloroquine (HCQ; $10 \mu \mathrm{M})$ or vehicle (ddH2O) for 18 hours or 7 days. Lysates were subjected to Western blotting for LC3 with $\beta$ actin as loading control. A representative Western blot following HCQ treatment for 7 days is shown ( $\mathrm{n}=2$ experimental replicates of independent wells of a differentiation). (B) Representative flow cytometry dot plots of SFTPC ${ }^{\mathrm{tdT} / \mathrm{WT}}$ and SFTPC ${ }^{1737 / d \mathrm{dT}}$ iAEC2s with or without HCQ $(10 \mu \mathrm{M})$ treatment and representative live-cell imaging of SFTPC $^{\text {tdT/WT }}$ and SFTPC ${ }^{173 T / d T T}$ HCQ-treated alveolospheres (bright-field/tdTomato overlay) showing maintenance of the $\mathrm{AEC} 2$ program (mean $\pm \mathrm{SD}$ is shown; $\mathrm{n}=3$ experimental replicates of independent wells of a differentiation). Scale bars: $500 \mu \mathrm{m}$. (C) Representative bioenergetics bar graphs plotted as OCR/ECAR ratios under basal conditions or following addition of oligomycin $(4.5 \mu \mathrm{M} / 1)$ or FCCP $(1 \mu \mathrm{M} / 1)$ for SFTPC ${ }^{\mathrm{tdT} / \mathrm{WT}}$ (black circles) and SFTPC ${ }^{173 \mathrm{~T} / \mathrm{dT}}$ (purple squares) iAEC2s with or without HCQ $(10 \mu \mathrm{M})$ treatment for 7 days show a significant reduction in OCR/ECAR ratio only in $\mathrm{SFTPC}^{\mathrm{I73T/dT}}$ iAEC2s treated with HCQ ( $\mathrm{n}=2$ independent experiments). ${ }^{*} \mathrm{p}<0.05$, by unpaired, two-tailed Student's t-test. 
Figure S6. iPSC line characterization, related to STAR Methods. (A) Representative G-banding analyses (SPC2-ST-C11, SPC2-ST-B2, SPC2-CorrC1, SPC6, and SPC7 iPSC lines) showing normal (46XY or 46XX) karyotypes. (B) Representative immunofluorescence microscopy images showing expression of the pluripotency markers TRA-1-60, TRA-1-81, and SSEA-4 but not SSEA-1 (as expected for human pluripotent stem cells) in each indicated, undifferentiated iPSC line (ES Cell Characterization Kit, SCR001 EMD Millipore). 


\begin{tabular}{|c|c|c|c|}
\hline & SPC2 & SPC6 & SPC7 \\
\hline Age at presentation & Late infancy & 2 months old & Infancy \\
\hline $\begin{array}{l}\text { Presenting clinical } \\
\text { phenotype }\end{array}$ & $\begin{array}{l}\text { Failure to thrive and } \\
\text { respiratory failure requiring } \\
\text { prolonged mechanical } \\
\text { ventilation }\end{array}$ & $\begin{array}{l}\text { Progressive respiratory } \\
\text { difficulties, } \\
\text { gastroesophageal reflux, } \\
\text { vomiting of feeds, and } \\
\text { failure to thrive }\end{array}$ & $\begin{array}{l}\text { Respiratory symptoms } \\
\text { (details not available) }\end{array}$ \\
\hline CT chest & $\begin{array}{l}\text { Diffuse ground glass } \\
\text { opacities and fine reticular } \\
\text { interstitial infiltrates at } \\
\text { presentation progressed to } \\
\text { also include traction } \\
\text { bronchiectasis and } \\
\text { honeycombing (Figure 1B) }\end{array}$ & $\begin{array}{l}\text { Diffuse ground glass } \\
\text { opacities (Figure S1A) }\end{array}$ & $\begin{array}{l}\text { Bilateral ground glass } \\
\text { opacities and traction } \\
\text { bronchiectasis (Figure S1A) }\end{array}$ \\
\hline Clinical course & $\begin{array}{l}\text { Treated with corticosteroids } \\
\text { and weaned to nasal cannula } \\
\text { supplemental oxygen }\end{array}$ & $\begin{array}{l}\text { Started on continuous } \\
\text { supplemental oxygen and } \\
\text { treated with corticosteroids } \\
\text { and hydroxychloroquine }\end{array}$ & $\begin{array}{l}\text { Treated in childhood with } \\
\text { many years of } \\
\text { corticosteroids and } \\
\text { hydroxychloroquine and } \\
\text { listed for lung transplant but } \\
\text { not transplanted }\end{array}$ \\
\hline Outcome & $\begin{array}{l}\text { Progressive respiratory } \\
\text { failure requiring lung } \\
\text { transplantation in early } \\
\text { adolescence }\end{array}$ & $\begin{array}{l}\text { Has required gastrostomy } \\
\text { tube feeds and continuous } \\
\text { supplemental oxygen } \\
\text { through } 3 \text { years of age }\end{array}$ & $\begin{array}{l}\text { As a young adult, had a } \\
\text { forced vital capacity (FVC) } \\
\text { of } \sim 20 \% \text { predicted }\end{array}$ \\
\hline Other & $\begin{array}{l}\text { Lung explant histology } \\
\text { displayed interstitial } \\
\text { fibrosis, chronic } \\
\text { inflammation, and alveolar } \\
\text { remodeling (Figure 1C) }\end{array}$ & $\begin{array}{l}\text { Has had recurrent } \\
\text { admissions for respiratory } \\
\text { symptoms }\end{array}$ & Mother of SPC6 donor \\
\hline
\end{tabular}




\begin{tabular}{|c|c|c|}
\hline \multicolumn{3}{|c|}{$\begin{array}{l}\text { Table S3. Oligonucleotides (related to Key Resources Table) } \\
\text { TaqMan Gene Expression Assay Primer/Probe Sets }\end{array}$} \\
\hline SFTPC & Thermo Fisher Scientific & Hs00161628_m1 \\
\hline SFTPB & Thermo Fisher Scientific & Hs01090667_m1 \\
\hline SLC34A2 & Thermo Fisher Scientific & Hs00197519_m1 \\
\hline NAPSA & Thermo Fisher Scientific & Hs00362192_m1 \\
\hline $18 S$ & Thermo Fisher Scientific & 4318839 \\
\hline Oligonucleotide primers & \multicolumn{2}{|c|}{ Integrated DNA Technologies } \\
\hline SFTPC I73T CRISPR gRNA & This paper & 5' CAGGTTCTGGA GATGAGCAC 3' \\
\hline $\begin{array}{l}\text { SFTPC I73T Correction single- } \\
\text { stranded oligonucleotide }\end{array}$ & This paper & $\begin{array}{l}\text { ' } \\
\text { GGTGGCTCCATGCCCTTTCCCCAGGTTCTG } \\
\text { GAAATGTCAATTGGTGCGCCGGAAGCCCAG } \\
\text { CAACGCCTGGCCCTGAGTGAG 3, }\end{array}$ \\
\hline SFTPC I73T Correction Forward & This paper & 5' GTCGTGGTGAT TGTGGGAGCCCTG 3' \\
\hline SFTPC I73T Correction Reverse & This paper & 5' CTGGGCATACC CACCTGCTGGTAG 3' \\
\hline $\begin{array}{l}\text { SFTPC I73T Correction } \\
\text { Sequencing }\end{array}$ & This paper & 5' TATGGGGATGG GTACCACTGGCTG 3' \\
\hline tdTomato Targeting Forward & (Jacob et al., 2017) & 5' GGGTGAGTGAG CTGATTCGAG 3' \\
\hline tdTomato Targeting Reverse & (Jacob et al., 2017) & 5' TGACCTCCTCG CCCTTGCTCACCATG 3' \\
\hline Intact SFTPC Locus Forward & (Jacob et al., 2017) & 5' CTACGGACACA TATA AGACCCTGGTC 3' \\
\hline Intact SFTPC Locus Reverse & This paper & 5' CAAATAGCCAC CATTCGGACACTC 3' \\
\hline Intact SFPTC Locus Sequencing & This paper & 5' TATGGGGATGG GTACCACTGGCTG 3' \\
\hline STEMCCA Excision Forward & (Somers et al., 2010) & 5' GGAACTCTTGT GCGTAAGTCGATAG 3' \\
\hline STEMCCA Excision Reverse & (Somers et al., 2010) & 5' GGAGGCGGCCC AAAGGGAGATCCG 3' \\
\hline $\begin{array}{l}\text { Puromycin Resistance Cassette } \\
\text { Forward }\end{array}$ & (Jacob et al., 2017) & 5'ATGACCGAGTA CAAGCCCACG 3’' \\
\hline $\begin{array}{l}\text { Puromycin Resistance Cassette } \\
\text { Reverse }\end{array}$ & (Jacob et al., 2017) & 5' TCAGGCACCGG GCCTGC 3’ \\
\hline Mki67 Forward & This paper & 5' ACCATCATTGACCGCTCCTT 3' \\
\hline Mki67 Reverse & This paper & 5' GCCCTGATGAGTCTTGGCTA 3' \\
\hline Top2a Forward & This paper & 5' TACAGTGCTCAACCTCTGACG 3' \\
\hline Top $2 a$ Reverse & This paper & 5' GGGATCTCGTGTTGGGAAGG 3' \\
\hline
\end{tabular}

